This item was submitted to Loughborough's Research Repository by the author.

Items in Figshare are protected by copyright, with all rights reserved, unless otherwise indicated.

\title{
Energy recovery from landing aircraft
}

PLEASE CITE THE PUBLISHED VERSION

PUBLISHER

(C) Shamsul Aizam Zulki

LICENCE

CC BY-NC-ND 4.0

REPOSITORY RECORD

Zulkifli, Shamsul A.. 2012. "Energy Recovery from Landing Aircraft". figshare. https://hdl.handle.net/2134/10843. 
This item was submitted to Loughborough's Institutional Repository (https://dspace.lboro.ac.uk/) by the author and is made available under the following Creative Commons Licence conditions.

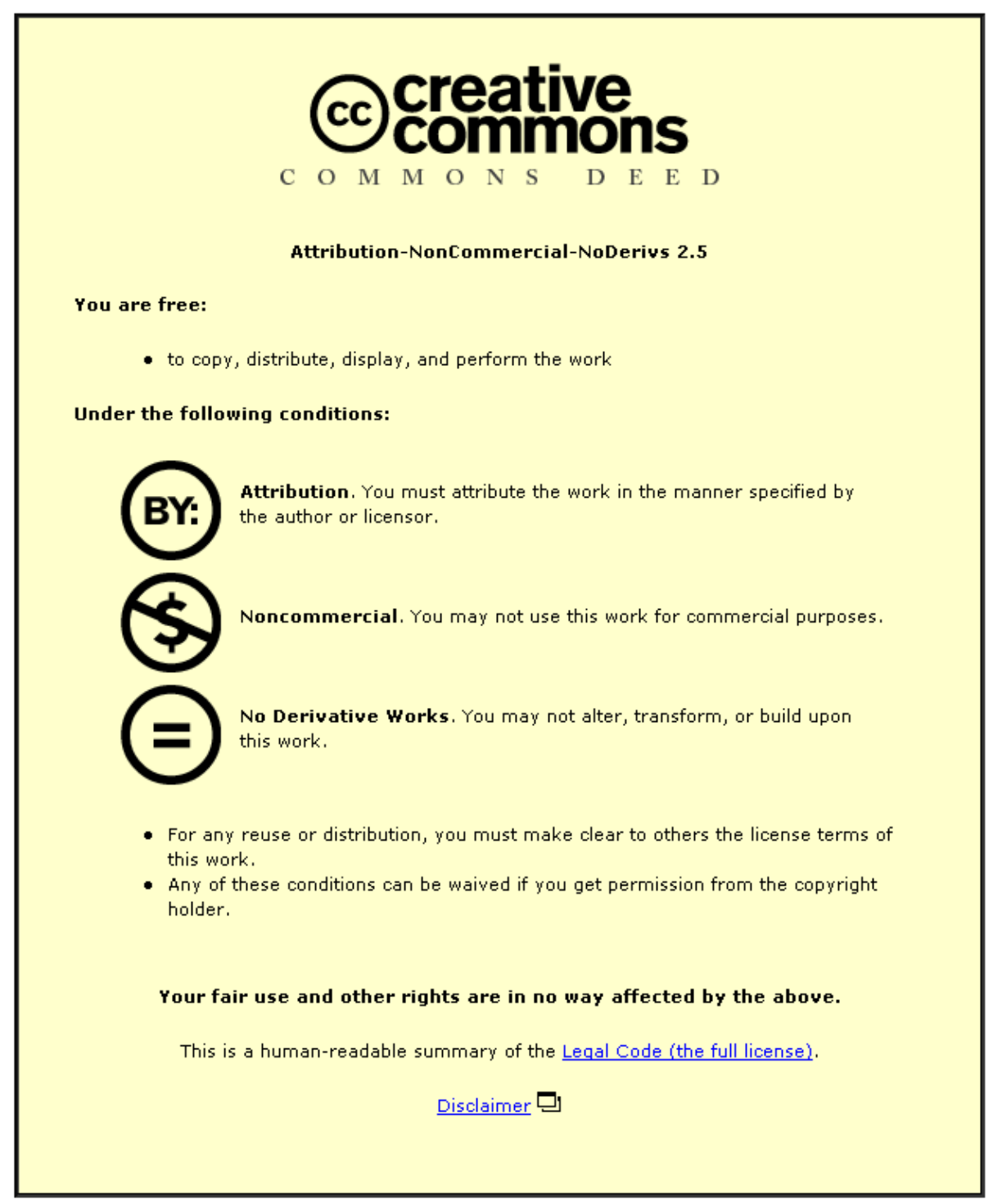

For the full text of this licence, please go to: http://creativecommons.org/licenses/by-nc-nd/2.5/ 


\title{
Energy Recovery From Landing Aircraft
}

\author{
by
}

\author{
Shamsul Aizam Zulkifli
}

\begin{abstract}
A Doctoral thesis
submitted in partial fulfilment of the requirements for the award of

Doctor of Philosophy

of Loughborough University
\end{abstract}

August 2012

(C) by Shamsul Aizam Zulkifli 2012 
to my family....Emma, Sarah and Syahira 


\begin{abstract}
Currently, renewable energy sources are the main driver for future electricity generation. This trend is growing faster in the developed countries in order to reduce the green house effect and also in response to the limited supply of oil, gas and coal which are currently the major sources for electric generation. For example, the main renewable energy sources are from wind energy and solar energy but these energies are only available to those countries that are exposed to these resources. In this thesis an alternative energy source is investigated where it can be generated from the moving objects or in form of kinetic energy. The idea is to convert the kinetic energy during landing aircraft into electrical energy which it can also be stored and transferred to the existing electrical network. To convert this kinetic energy to electrical energy, the linear generator (LG) and uncontrolled rectifier have been used for energy conversion. The LG have been modelled in 3-phase model or in $d q$ model and combined with the diode rectifier that is used to generate the dc signal outputs. Due to the uncontrolled rectifier the electrical outputs will have decaying amplitude along the landing time. This condition also happen to the LG outputs such as the force and the power output. In order to control these outputs the cascaded buck-boost converter has been used. This converter is responsible to control the output current at the rectifier and also the LG output power during landing to more controllable power output. Here, the $H^{\infty}$ current control strategy has been used as it offers a very good performance for current tracking and to increase the robustness of the controller. During landing, huge power is produced at the beginning and when the landing time is increased, the generated input power from LG is reduced to zero. Due to this, the energy storage that consists of ultracapacitor, bidirectional converter and boost converter are used in order to store and to release the energy depends on the input power source and load grid power. The voltage proportional-integral (PI) control strategy has been used for both the converters. The last part is to transfer the energy from the source and at the ultracapacitor to the load by using the inverter as the processing device. The power controller and current controller are used at the inverter in order to control the power flow between the inverter and the grid. This is when the reference power is determined by the load power in order to generate the reference currents by using the voltage oriented controller (VOC), while the $H^{\infty}$ current controller is used to regulate the inverter currents in order to inject the suitable amount of current that refer to the load power. Finally, a complete energy recovery system for landing aircraft with the grid connection have been put together to make the whole system to be as a new renewable energy source for the future electricity generation.
\end{abstract}




\title{
Acknowledgements
}

\author{
Bismillah Ir-Rahman Ir-Rahim
}

First of all, I would to thank the almighty GOD that gives me strengh, good health and his blessing to complete my $\mathrm{PhD}$ work far away from my home country. At the same time, I would also like to thank my supervisor, Professor Qing-Chang Zhong for his encouragement, guidance and great support during the course of my $\mathrm{PhD}$ study, not just about the research but also for teaching me a life lesson on how to balance life between family and study. He was also always ready to discuss the details of this work and share his expertise in control theory and power electronics, which made it possible to complete this work.

Here, I also would like to thank Professor Wen-Hua Chen, for his support during my last stage of my PhD study in order to complete my thesis. To all my colleagues, Dr Xin Cao, Dr Long, Dr Tomas Hornik, Zhenyu Ma, Max Ming and Olivia, who shared the knowledge that they have from the beginning until the end of my $\mathrm{PhD}$ journey, and which made this work enjoyable.

Last but not least, I would like to thank my wife, Mrs Emma Suzlina Mokhtar for her continuous morale support and sacrifices that she made during 4 years away from her family.

Finally, I would to thank again all those who have made my $\mathrm{PhD}$ study successful. God bless you all. 


\section{Contents}

1 Introduction $\quad 1$

1.1 Motivation ............................. 1

1.2 Aim and Objectives ..................... 2

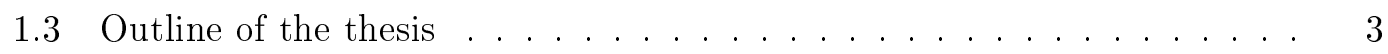

1.4 Contribution of the research . . . . . . . . . . . . . . . . . . 4

1.4.1 Energy conversion .................. 4

1.4.2 Energy storage ..................... 5

1.4.3 Energy transfer .................... 5

1.5 Concluding remarks ........................ 6

2 Literature Review $\quad 7$

2.1 Alternative energy sources as renewable energy sources . . . . . . . . 7

2.2 Introduction to distributed generation (DG) for renewable energy sources 8

2.3 Energy conversion ....................... . . 10

2.3.1 Linear synchronous machine in energy conversion . . . . . . . . 11

2.3.2 DC-DC converter as power conditioning in energy conversion . . 15

2.4 Energy storage . . . . . . . . . . . . . . . . . . . . 17

2.4.1 Energy storage elements . . . . . . . . . . . . . . 18

2.4.2 Energy storage with bidirectional converter . . . . . . . . 19

2.4.3 Boost converter .................... 20

2.5 Energy transfer ......................... 20

2.5.1 Filter component at the energy transfer part . . . . . . . 20

2.5.2 Synchronisation of the inverter with the grid source . . . . . . 21

2.5.3 Power flow between the inverter and the grid source . . . . . . 22

2.5.4 Control scheme for inverter-side converter . . . . . . . . . 22

2.5.4.1 Current control for the inverter .......... 23

2.5.4.2 Power control technique at the inverter . . . . . . . 24

$2.6 H^{\infty}$ control theory . . . . . . . . . . . . . . . . . . . . 26

2.6.1 $H^{\infty}$ control application in dc-dc converters . . . . . . . . . 27 
2.6.2 $H^{\infty}$ control application in the inverter-grid connection . . . . . 27

2.6.3 System stability . . . . . . . . . . . . . . . . . 28

2.7 ERLA configuration in the renewable energy application . . . . . . . 29

2.8 Concluding remarks . . . . . . . . . . . . . . . . . 30

3 Energy conversion:Modelling of the linear generator 32

3.1 Potential kinetic energy generated during normal landing . . . . . . . . 32

3.2 Overview of a 3 -phase synchronous generator . . . . . . . . . . . 33

3.3 Linear generator and diode rectifier for electrical power generation . . . 35

3.4 Modelling of the linear generator . . . . . . . . . . . . . . 37

3.4.1 Linear generator model in 3-phase system . . . . . . . . . . 37

3.4.2 Linear generator model in $d q$ transformation model . . . . . . . . 42

3.5 Simulation results . . . . . . . . . . . . . . . . . . . . 44

3.5.1 Linear generator with 3 -phase model . . . . . . . . . . 45

3.5.2 Linear generator with $d q$ transformation model . . . . . . . . . . 48

3.6 Concluding remarks $\ldots \ldots \ldots \ldots$

4 Energy conversion: The $H^{\infty}$ current control design for generated out$\begin{array}{ll}\text { puts } & 51\end{array}$

4.1 The model and parameters calculation of the CBB converter . . . . . 51

4.2 Design of $H^{\infty}$ current control for the CBB converter . . . . . . . . . 55

4.2.1 Description of the control scheme . . . . . . . . . . 56

4.2.2 Switching logic in order to have the buck or boost mode control . 56

$4.2 .3 \quad H^{\infty}$ current controller design . . . . . . . . . . . . . 57

4.2.4 Determining the stability performance for the $H^{\infty}$ current controller 63

4.3 Simulation results . . . . . . . . . . . . . . . . . . 65

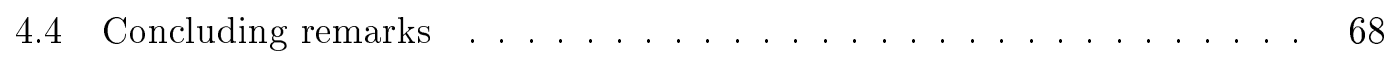

5 Energy storage: The dc-dc converters control for energy storage $\quad 69$

5.1 Modelling and control of the dc-dc converters for the energy storage . . 69

5.1 .1 The sizing of the ultracapacitor f . . . . . . . . 70

5.1.2 Control strategy for the bidirectional converter with the ultracapacitor ....................... 71

5.2 Modelling and control of the boost converter . . . . . . . . . . . . 73

5.2.1 Control strategy for boost converter . . . . . . . . . . . . 74

5.3 Simulation results . . . . . . . . . . . . . . . . . . . . . . 75

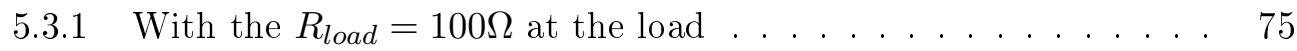

5.3.2 With the $R_{\text {load }}=150 \Omega$ at the load . . . . . . . . . 78

5.4 Concluding remarks . . . . . . . . . . . . . . . . . 81 
6 Energy transfer: VOC PI - $H^{\infty}$ power flow control 82

6.1 Description of the inverter at the grid connected circuit . . . . . . . 82

6.2 Description of controller model for the inverter . . . . . . . . . . 84

6.2 .1 VOC-PI for outer power loop . . . . . . . . . . . . 85

6.2.2 $H^{\infty}$ current control design for inner control loop . . . . . . . 86

6.2.2.1 State-space model of the inverter for $H^{\infty}$ control calculation . . . . . . . . . . . . . 86

6.2.2.2 $H^{\infty}$ formulation for current controller design $\ldots \ldots . \quad 88$

6.2.2.3 $H^{\infty}$ controller design $\ldots \ldots \ldots$. . . . . . . 90

6.2.2.4 Stability evaluation for the $H^{\infty}$ control . . . . . . . . 90

6.3 Simulation results . . . . . . . . . . . . . . . . . . . . . 92

6.3.1 With the reference power of $P^{*}=1000 \mathrm{~W}$ and reactive of $Q^{*}=$

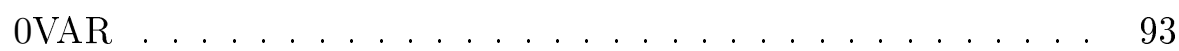

6.3.2 With $P^{*}=500 \mathrm{~W}$ from $t=0$ s to $t=3$ s and with real $P^{*}=1000 \mathrm{~W}$

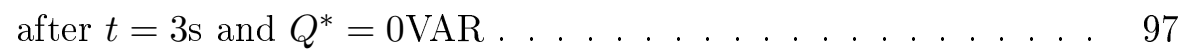

6.4 Concluding remarks . . . . . . . . . . . . . . . . . 100

$\begin{array}{llr}7 & \text { Conclusions and Future Work } & 101\end{array}$

7.1 Conclusions . . . . . . . . . . . . . . . . . . . . . . . . 101

7.2 Recommendations for future work . . . . . . . . . . . . . 103

$\begin{array}{ll}\text { A Publication papers and test rig schematic diagram } & 118\end{array}$

A.1 List of publications . . . . . . . . . . . . . . . . . 118

A.2 The schematic diagram for the test rig development . . . . . . . . . . 119

B Simulations test with different LCL value $\quad 120$

B.1 With the reference power of $P^{*}=1000 \mathrm{~W}$ and reactive of $Q^{*}=0 \mathrm{VAR}$. 120

B.2 With $P^{*}=500 \mathrm{~W}$ from $t=0$ s to $t=3$ s and with real $P^{*}=1000 \mathrm{~W}$ after

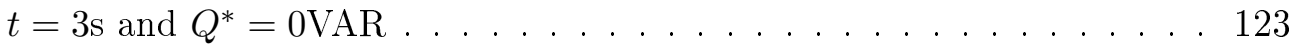

$\begin{array}{lr}\text { C List of symbols } & 125\end{array}$ 


\section{List of Figures}

2.1 Example of the renewable energy source in DG connection . . . . . . . 9

2.2 DGs in micro-grid connection . . . . . . . . . . . . . . . . 10

2.3 Topology of energy conversion in renewable energy source . . . . . . . . 11

2.4 Example of a linear generator f . . . . . . . . . . . . . . . 12

2.5 First technique of LG configuration in the ERLA application . . . . . 13

2.6 Second technique of LG configuration in the ERLA application . . . . . 14

2.7 Example of dc-dc converters that can be applied in energy conversion . 16

$\begin{array}{lll}2.8 \text { Circuit diagram of a cascaded buck-boost converter in energy conversion } & 17\end{array}$

2.9 Topology of energy storage in renewable energy source applications . . . 18

2.10 Topology of energy transfer process for renewable energy source . . . . 21

2.11 The block diagram of current control for energy transfer process in natural frame $(a b c) \ldots \ldots \ldots \ldots \ldots \ldots$

2.12 The block diagram of DPC control strategy . . . . . . . . . . . 25

2.13 The block diagram of power and current control in the energy transfer process . . . . . . . . . . . . . . . . . . 25

2.14 The standard $H^{\infty}$ optimal control formulation $\ldots \ldots \ldots$

2.15 The stability block for $\left\|T_{y u}\right\|$ in $H^{\infty}$ control . . . . . . . . . . . . 28

2.16 Overview of an energy recovery system for landing aircraft $\ldots . . . .30$

3.1 The block diagram of the energy conversion part . . . . . . . . . 33

3.2 The circuit diagram of the 3-phase synchronous generator equivalent model 34

3.3 The direction of magnetic field between the rotating machine and LG in one full pitch . . . . . . . . . . . . . . . . . 35

3.4 The block diagram of energy conversion without the dc-dc converter . . 37

3.5 The ideal three-phase generator in rotating mode . . . . . . . . . . 38

3.6 The model of linear machine in simulink block diagram for 3 phase analysis 42

3.7 Representing the direct-axis winding of synchronous generator after the dq transformation . . . . . . . . . . . . . . . . 43

3.8 The model of linear machine in simulink block diagram for $d q$ analysis . $\quad 45$

3.9 Simulation results for 3-phase linear generator model at the diode rectifier 46 
3.10 Simulation results for 3-phase linear generator model at LG outputs . . 47

3.11 Generated power during landing for 3 -phase model . . . . . . . . . . 47

3.12 Simulation results for dq linear generator model at the diode rectifier . 48

3.13 Simulation results for dq linear generator model at LG outputs . . . . . 49

3.14 Generated power during landing for $d q$ model $\ldots$. . . . . . . . . . . . 49

4.1 The circuit diagram of CBB converter in ERLA system . . . . . . . . . 52

4.2 The current control strategy for CBB converter . . . . . . . . . . . 53

4.3 The $H^{\infty}$ current control applied to the CBB converter . . . . . . . . 56

4.4 The block diagram of logic sequence used in the CBB converter for boost or buck mode operation . . . . . . . . . . . . . . . . 57

4.5 The CBB with the $\mathrm{H}^{\infty}$ control scheme . . . . . . . . . . . 58

4.6 The block diagram of the $H^{\infty}$ current control scheme in CBB converter . 58

4.7 The block diagram of the CBB converter . . . . . . . . . . . . 59

4.8 The complete structure for CBB converter with the inputs and outputs in $H^{\infty}$ control model . . . . . . . . . . . . . . . . 60

4.9 The singular analysis bode diagram . . . . . . . . . . . . 65

4.10 Simulation results at LG output . . . . . . . . . . . . . . . 66

4.11 Simulation results when $H^{\infty}$ current control applied to the CBB converter 67

4.12 The tracking error current $\left(I_{s}^{*}-I_{s}\right) \ldots \ldots \ldots 6$

5.1 The block diagram of the energy storage in an ERLA system . . . . . 70

5.2 The bidirectional converter with voltage control at the dc-link . . . . . 72

5.3 The boost converter circuit in energy storage . . . . . . . . . . . . . 73

5.4 The voltage control for boost converter . . . . . . . . . . . . . . 74

5.5 Simulation results at energy conversion outputs . . . . . . . . . . 76

5.6 Simulation results at the energy storage part $\left(R_{\text {load }}=100 \Omega\right) \ldots \ldots$. . . 77

5.7 The ultracapacitor outputs when the load is $R_{\text {Load }}=100 \Omega \ldots \ldots 78$

5.8 Simulation results at energy conversion outputs . . . . . . . . . . . 79

5.9 Simulation results at the energy storage part $\left(R_{\text {load }}=150 \Omega\right) \ldots \ldots$. . 80

5.10 The ultracapacitor outputs when the is $R_{\text {Load }}=150 \Omega \ldots \ldots$. . . . 80

6.1 Equivalent single phase inverter . . . . . . . . . . . . 83

6.2 The control strategy for inverter-grid connection in ERLA energy transfer

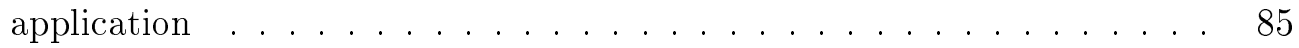

6.3 The block diagram of power control with VOC control strategy . . . . 86

6.4 The $H^{\infty}$ current control for the inner loop controller . . . . . . . . 87

6.5 Single phase representation of the inverter . . . . . . . . . . . . 87

6.6 The block diagram to formulate the $H^{\infty}$ control theory for current controller 89 
6.7 The block diagram of stability structure for current control _ . . . . . 90

6.8 Simulation outputs at energy conversion part when all the parts combined together . . . . . . . . . . . . . . . . . . 93

6.9 Simulation outputs at energy storage part when all the parts combined together . . . . . . . . . . . . . . . . . . 94

6.10 Simulation outputs at energy transfer when $\mathrm{P}$ is set to $P^{*}=1000 \mathrm{~W}$. . 95

$6.11 H^{\infty}$ current controllers response in the ERLA system . . . . . . . . . . 96

6.12 Synchronisation mechanism between grid voltage $v_{g}$ and inverter voltage $v_{i} 96$

6.13 Simulation outputs at energy conversion part when all the parts combined together . . . . . . . . . . . . . . . . . 97

6.14 Simulation outputs at energy storage part when all the parts combined

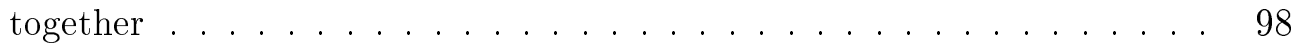

6.15 Simulation outputs at energy transfer when P has a step response from $P^{*}=500 W$ to $1000 W \ldots \ldots \ldots \ldots \ldots$

$6.16 H^{\infty}$ current controllers response in the ERLA system . . . . . . . . 100

A.1 Schematic diagram for the rig f . . . . . . . . . . . . . 119

B.1 Outputs generated at the energy conversion . . . . . . . . . . . 121

B.2 Outputs generated at the energy storage . . . . . . . . . . . . 121

B.3 Outputs generated at the energy transfer . . . . . . . . . . . . 122

B.4 Outputs generated at energy conversion . . . . . . . . . . . 123

B.5 Outputs generated at the energy storage . . . . . . . . . . . . 124

B.6 Outputs generated at the energy transfer . . . . . . . . . . . . . . . 124 


\section{List of Tables}

3.1 Parameters for the $\mathrm{LG} \mathrm{model} \mathrm{\ldots ...} \mathrm{.} \mathrm{.} \mathrm{.} \mathrm{.} \mathrm{.} \mathrm{.} \mathrm{.} \mathrm{.} \mathrm{.} \mathrm{.} 45$

4.1 Parameters of the CBB converter . . . . . . . . . . . 65

5.1 Parameters to calculate the inductance and capacitance at boost converter 73

5.2 Parameters value for the energy storage . . . . . . . . . . . . 75

6.1 Design parameters . . . . . . . . . . . . . . . . . . . . 88 83

6.2 LCL filter values . . . . . . . . . . . . . . . . . . . 84

B.1 With the different value for LCL filter . . . . . . . . . . . . . . . 120 


\title{
List of Abbreviations
}

\author{
ac alternating current \\ CB Circuit breaker \\ CBB Cascaded buck-boost \\ dc direct current \\ dq direct-quadrate
}

ERLA Energy Recovery From Landing Aircraft

FIT Feed In Tariff

G plant

K compensator

LG Linear generator

PI proportional-integral

PLL Phase locked loop

PWM Pulse width modulation

RHI Renewable Heat Incentive

VOC voltage oriented control 


\section{Chapter 1}

\section{Introduction}

\subsection{Motivation}

Electrical energy is the fundamental and main energy source which makes the world move forward. This energy can be generated from heat, transportation and natural resources such as coal, oil, gas, wind, solar and etc. Most electrical energy that generates today is from coal, oil and gas sources where its have been used for over a century ago. Currently these sources are reducing faster than before and it has triggered a new electrical energy exploration that the supply are coming from the renewable energy sources. It needs to be done quickly and continuously in order to meet today increasing energy demands. Most of the developed countries have explored these renewable energy sources such as wind power, solar power, tidal power and have put this task in their mainstream growth, and also included it in the annual budget to encourage people to exploit these free resources.

For example, the UK government has developed the energy policies to meet, to reduce and to promote the energy consumption and also to increase the usage of the renewable sources as a main electrical energy supply. It suggests that the electricity generation from renewable energy sources needs to increase to $30 \%$ by 2020 compared to $6.7 \%$ [1] of the generation today which includes both centralised and small-scale generation. In doing so, the government will spend around 660 million pounds [1] to achieve this target. On the other hand, the UK government has also agreed to create a system that is called the Renewable Heat Incentive (RHI) and the Feed In Tariff (FIT) for electricity generation less than 5MW, which is suitable for organisations, businesses, communities and individuals that are not involved in the electricity market in order to supply electricity to the main power grid [1]. These initiatives mean that the power system configuration is undergoing tremendous changes in order to be able to accept power generation from renewable energy resources. The other reason why the government has put these initiatives into practise is to reduce the levels of carbon dioxide 
$\left(\mathrm{CO}_{2}\right)$ released to the atmosphere that causes the global warming. This situation has caused the Earth to become warmed by about 0.8 degrees per decade compared to the last 30 years which about 0.2 per decade [2]. As a result of this, the UK in 2007 has seen the warmest year compared to the previous decade. This issue needs to be solved and it has driven more renewable energy technology into action.

Alternative energy resources are also part of the renewable energy sources, and include the sources from waste energy such as kinetic energy which have no undesired consequences after generation. From the energy law, energy cannot be eliminated but it can be changed to another form of energy. Due to this concept regenerative braking has been introduced into vehicles. This is where the kinetic energy can be changed to electrical energy when the vehicle is slowing down. The energy produced here is small due to the low inertia and is only suitable for use in the vehicle operations such as initial start or small mechanical works. From this concept, huge amounts of energy can be produced and changed if high inertia can be stored in the vehicle. This has lead to using aircraft during landing as a source which stores huge kinetic energy where currently has been wasted as heat.

As a result, this research is undertaken in order to recognise, to understand and to process this alternative energy source that is generated from the landing aircraft and changed to electrical energy in order to increase the power generation target set for the renewable energy sources and at the same time reduce the greenhouse effect on the environment.

\subsection{Aim and Objectives}

The aim of this research are to evaluate the feasibility of converting the kinetic energy from the landing aircraft as a alternative energy source to electrical energy, and to supply this energy back to the electrical network. In order to achieve this aim, four objectives are defined:

1. To study the components that can be used for converting, storing and transferring kinetic energy to electrical energy;

2. To change the generated power during landing by controlling the LG current in order to minimise the uncomfort condition to the passenger by maintaining the force generated from the LG;

3. To develop the control strategies that can be used to control the generated power, to allow the power flow between the energy components and to transfer the generated power to the load; 
For objective 1, it can be done by determine a suitable generator, the storage element and the power processing devices that can be used in order to change the kinetic energy to electrical energy. The arrangement of each devices is based on the distribution generation connection which is suitable to transfer the energy from the renewable energy sources to the existing electrical network. The second objective can be achieved by finding the relationship between the generated power and the output current of the LG which is determined by the landing speed. This relationship will minimise the operation cost because no speed sensor will be used in order to control the generated power during landing. At the same time it can also control the braking force generated by the LG which determines the comfort of the passengers in the aircraft.

Finally, the selection of the control strategy needs to be conducted based on objectives 1 and 2 in order to have the power flow from the alternative energy source to the load or electrical grid. The chosen controller also needs to respond to the variable magnitude inputs, disturbances and gives robust conditions.

\subsection{Outline of the thesis}

The concept of converting kinetic energy to electrical energy involves several parts, such as the energy conversion part, the energy storage part and the energy transfer part. The energy conversion part is described in two chapters which are Modelling of the linear generator for energy conversion and The $H^{\infty}$ current control design for the energy conversion. There is one chapter for the energy storage and one chapter for the energy transfer. All of these are organised as follows.

Chapter 2 is about the literature review where it explains the functions of the components and the control strategies that will be used to develop the ERLA configuration. As an addition, the $H^{\infty}$ control theory has been summarised in order to help to build the control application for the dc-dc converters, and the inverter that will be used in this work.

Chapter 3 is about the energy conversion process that is related to the energy conversion from kinetic energy to electrical energy by using the LG and the rectifier. This linear generator is been modelled in a 3-phase model and in a $d q$ transformation model to behave as the LG machine. It is used to see the electrical and the mechanical outputs response for the LG when the landing speed as the input parameter. The LG models have been modelled in the MATLAB simulink software where both models show the same results. This indicates that both models can be used as the LG model in the ERLA system network.

Chapter 4 covers the control part of the energy conversion, and at the same time will control the LG outputs. The main function for this part is to control the deceleration, 
force and the shape of the generated power by only controlling the input current at the converter side. These parameters have been given more attention in order to determine the success of the control strategy. The cascaded buck-boost converter with the current control topologies is designed by using the $H^{\infty}$ control theory. This is where the converter can respond to the wide input range of voltage that is coming from the LG outputs.

The energy storage section that consists of the energy storage element and the dcdc converters are explained in Chapter 5. In this chapter, the amount of energy that needs to be stored in the storage elements has been calculated and it depends on the maximum generated power. The bidirectional and boost converters have been selected in order to achieve the target for these converters with the proportional-integral control as the control strategy.

In order to transfer the energy from the source and with the energy from the storage element to the electrical grid network, a 3-phase inverter has been used which is explained in Chapter 6. This chapter also includes the design steps for the LCL filter calculation. The $H^{\infty}$ current control has been used to deal with the current control and the voltage oriented control-proportional-integral (VOC-PI) for energy transfer process. Attention is paid at the power output transfer in response to the power changing at the load in order to determine the effectiveness of the control strategy that has been proposed.

Finally, the main conclusions of the thesis are summarised and possible future works are proposed in Chapter 7 .

\subsection{Contribution of the research}

In this research, the contribution to knowledge is based on the control strategies applied to the ERLA system in order to transfer the kinetic energy from the landing aircraft to the electrical energy at the grid.

\subsubsection{Energy conversion}

Here, the main contribution of the ERLA is to change the instantaneous power which has exponential decay graph where it is normally generated during the landing, to a more useful information/graph that can be used to determine the average power with a linearly decreasing graph. To obtain this condition, the generated force from the linear generator must be constant, which will create the linear decreasing power graph [3]. This force also relates to the braking force which is applied to the passenger. During normal landing, the aircraft generates its own force while the braking force is applied 
in the opposite direction to the landing force. By maintaining this braking force the passengers will not feel any discomfort during landing. In order to control this force, the deceleration control needs to be used. For the ERLA system, no control action will be used at the LG in order to reduce the number of the controllers applied to the system. This action is taken by the dc-dc converter, which is one of the power processing devices in the energy conversion part. The current control strategy has been used as the controller, due to the relationship between the input current, the force, the power and the deceleration. This is when the input current is controlled at the dc-dc converter, and at the same time it is capable of controlling the force and deceleration at the linear generator. For the current controller design, the $H^{\infty}$ control theory has been used as the current control strategy. This is because, it is capable of giving small tracking error between the feedback input current at the dc converter with the reference current and it is also able to respond to the wide output voltage generated from the LG.

\subsubsection{Energy storage}

Energy storage is used to temporarily store the energy when the source energy is higher than the load, and to discharge the energy when the load required more energy than the main source can supply. For the ERLA, the energy conversion target is to deal with the input current but not the output voltage at the dc-link. If there is no voltage control applied at the dc-link, the energy storage can only store the energy without having the ability to discharge the energy back to the load when it is required. Due to this, the voltage control at the dc-link connection between the energy conversion and the energy storage can be applied at the bidirectional converter. In the meantime, this controller will give freedom to the storage element to store how much power regarding to the rated energy. In doing so, the proportional-integral control has been used due to the maturity of the controller.

\subsubsection{Energy transfer}

It is used to transfer the generated energy from the landing aircraft and the storage element to the grid. The voltage oriented control has been used for the power control strategy and it is combined with the $H^{\infty}$ control theory for the current controller in order to have the energy flow between them. The reason why the $H^{\infty}$ control theory has been selected is because the compensator value that has been designed is included the LCL filter components in the compensator calculation. By including these components, the controller that will be designed is more robust where it has a large bandwidth, and is capable of responding to the wide range of LG outputs. At the same time, it can also minimise the tracking error. Finally, all the parts are combined together to model the 
complete ERLA system and it shows that the kinetic energy from the landing aircraft can be converted, stored and transferred by using the proposed controller given in this work.

\subsection{Concluding remarks}

The main motivation that triggers this work is to increase the capacity of the renewable energy sources in order for them to be the main sources of electricity generation in the future. By using more and more renewable energy sources the dependency on oil and gas sources can be reduced dramatically and at the same time this can reduce the $\mathrm{CO}_{2}$ emission to the air and make the environment more healthy. The alternative energy which is the kinetic energy that has been left behind can be the new energy source for renewable energy source. This is because more heavier and more high speed systems have been built for vehicles today, which is the main source for kinetic energy. Currently, this energy has been wasted as heat without any action to recover it. This has lead to the aim of this research is to convert the kinetic energy from high speed vehicles such as aircraft when it start to landing to electrical energy. To achieve this aim, several objectives have been listed in order to capture, to store and to transfer the energy to the existing electric network. While conducting this research several contributions have been found out but the main contribution of this research is the relationship between the input current at the dc converter with the force and the power output for the LG whichcan reduce the number of controllers that will be used in ERLA system. This is achieved by using only one controller at the converter side which is capable of controlling the LG outputs. 


\section{Chapter 2}

\section{Literature Review}

\subsection{Alternative energy sources as renewable energy sources}

Alternative energy sources can be divided into six categories which are chemical energy, thermal energy, kinetic energy, nuclear energy, rotational energy and solar energy. Chemical and nuclear energy are created due to chemical reactions and require a combustion to generate the electricity. These energies create air pollution and require a control environment to operate. However, these energy sources can generate high amounts of electric power with continuous supply. Thermal, rotational and solar energy are natural sources of energy that can be found in the environment and are free sources. The limitation for these energies is due to the location of the source. For example, wind turbines are most likely to be used in north European countries such as Denmark, the UK and Norway which are exposed to strong windy conditions in order to generate the electricity, while countries that are exposed to high solar radiation such as Mexico, Australia and the United States use more solar energy source to supply electricity to the electric network. The electricity production from these sources is not constant and needs a power tracking function to maximise the electricity production. Regarding [4, 5] the other type of alternative energy comes from waste energy. This waste energy can be obtained from food waste or biomass waste. Kinetic energy can also be categorised as a waste energy. This kinetic energy can be found when there is a movement of an object from one point to another point. This energy can be found in small amounts from water drops or in large amounts as in high speed vehicle.

According to the Defense Advanced Research Projects Agency (DARPA), humans can generate around 1 to 2 watts of energy when they walk. This indicates that humans can also generate electricity. Research has been conducted by [6], which converts walking energy to electric energy using the microgenerator made for military purposes. As is known, a normal soldier in service at the war zone will carry around $7.5 \mathrm{~kg}$ to $13 \mathrm{~kg}$ of 
battery to power up military equipment such as night vision goggles, radio communication and other electric equipments [6]. By replacing the battery with a microgenerator, the weight carried by the soldier is reduced dramatically. Electrical energy is produced due to the inertial body mass and the walking speed of the soldier. Imagine when huge amounts of mass found in high speed objects, it will create huge amount of kinetic energy.

Here, this concept has been expanded to regenerative braking for vehicles. For vehicle purposes, it converts kinetic energy to electrical energy when the vehicle starts to slow down $[7,8]$. In this mechanism, the motoring operation is changing to generator operation in order to store the energy inside the battery and it will be used for the next acceleration cycle of the vehicle. This application has been applied in the hybrid car concept such as in the Toyota Prius and other new car generations in order to reduce the $\mathrm{CO}_{2}$ emission to the environment.

Looking at the bigger picture, for huge energy production, this concept can be applied to the airline industries. This is when the kinetic energy created during the landing aircraft is converted to electrical energy $[9,10]$. At the same time, this electrical energy can also be stored and used for the taxiing or takeoff process where it will reduce the pollution at the airport, such as noise pollution. It will also reduce the fuel cost since the amount of fuel burning during takeoff can be minimised and the aircraft can carry less fuel for long distances travel. In order to harvest this potential energy from the landing aircraft, the configuration of the distributed generation structure can be used to change, to store and to transfer this kinetic energy to electrical energy for usage at the load side.

\subsection{Introduction to distributed generation (DG) for renew- able energy sources}

DG is an electric power generation which is located near to the load/ electrical grid in order to reduce the losses along the transmission line that happen in power generation by the synchronous generator, or to improve the voltage or current at the distribution network. This configuration is suitable for renewable energy production where the power generated can be connected directly to the load without having transmission losses. To convert the renewable energy to electrical energy, a power conditioning process is needed. It uses several power converters as shown in Figure 2.1. It consists of an acdc converter, a dc-dc converter and a dc-ac converter. The advantages of using these converters are: they are easy to develop, and the control methods are easy to model with regards to the control objective of the converters. The generator is used to change 
the mechanical inputs to electrical outputs. Figure 2.1 shows an example of renewable energy sources that are used the DG structure. The energy storage system is used next to the converters, and the generator, in order to provide a place to store the excess energy at the beginning and also to supply the power when the load demand is higher than the amount of power that can be provided by the source. The filter and the transformer in Figure 2.1 are used to connect the DG to the grid without affecting the voltage and current at the grid. The filter, such as inductor (L), or inductor-capacitor (LC), or inductor-capacitor-inductor (LCL), can be applied to reduce the injected harmonic current to the grid by the inverter.

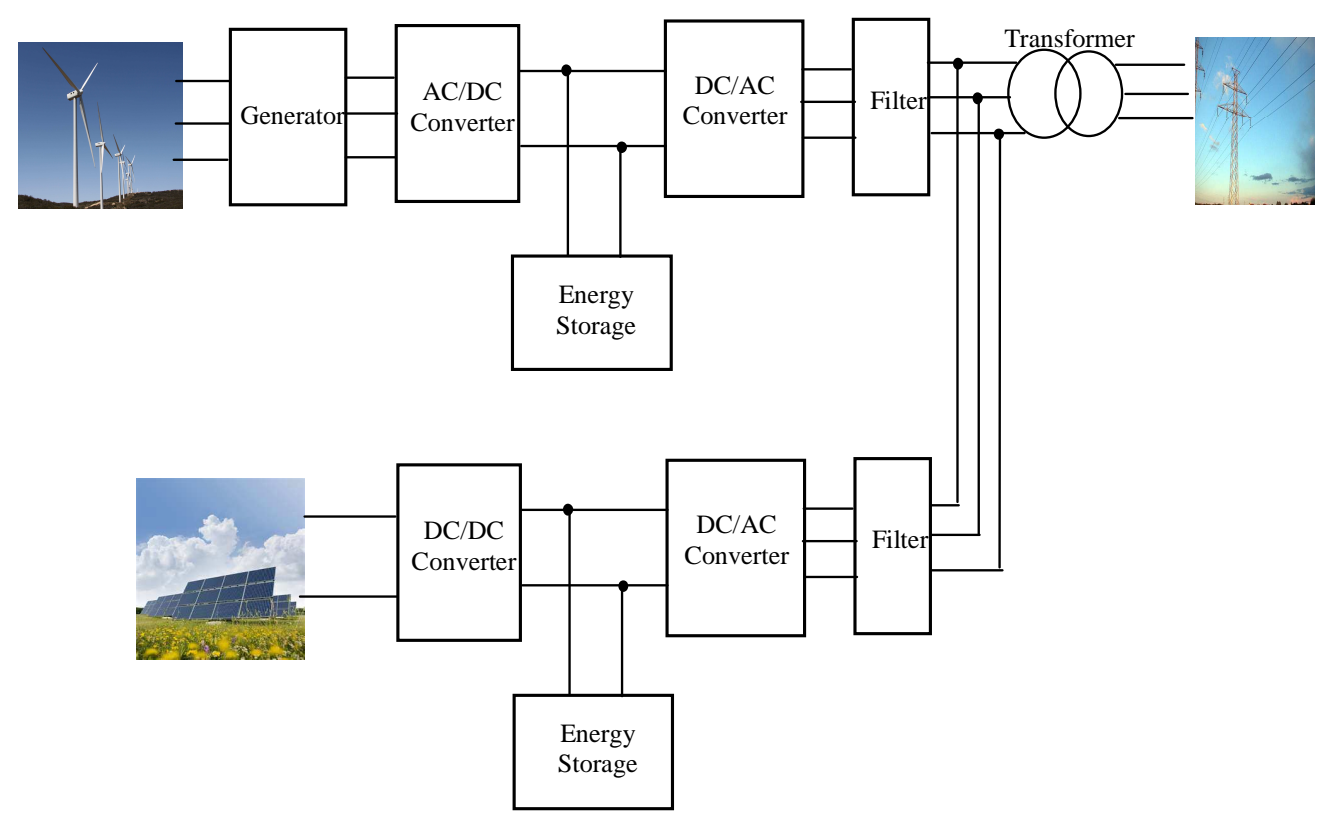

Figure 2.1: Example of the renewable energy source in DG connection

The examples of renewable energy sources that can be applied to the DG are wind energy, solar energy, fuel cell energy and alternative energy sources. These sources can be separated into two types of electrical output generation: the ac output source or the dc output source. Figure 2.1 shows these two kinds of electrical output. The only difference between them is that the generator block is used for ac output generation and not for dc output generation. In order to harvest the energy from renewable sources, power tracking control is needed, and it is a challenging task for the researchers because different sources need different methods of control. For example, wind and solar energy require maximum point power tracking in order to generate the maximum electrical output power, while for the wave energy this is determined by the motion of the point absorber [11]. 
As is known, the power generated from renewable sources is currently lower than the power generated from the synchronous generator. Because of this, the DG cannot be operated alone. By connecting several of them to the existing distribution network, a micro-grid can be formed as shown in Figure 2.2. This micro-grid will give a continuous and same amount of power supply likes the synchronous generator by connecting other renewable energy sources that are placed in the electrical network. The circuit breaker $(\mathrm{CB})$ is used to disconnect or to connect other power sources to the existing electrical network. For a single DG configuration, it is more suitable to use at the remote area where the electricity network is not available or difficult to reach by the transmission network.

Other than grid connection, the DG can also be connected directly to the load or can be as the main power source to the load. This kind of DG connection is known as the islanded mode. The islanded mode is basically used for small scale power supply while at the same time it is able to reduce the amount of carbon emission to the environment [12]. To understand the DG's operation under the renewable energy configuration more clearly, the DG system can be separated into three parts. The first part is the energy conversion, the second part is known as the energy storage, followed by the energy transfer part.

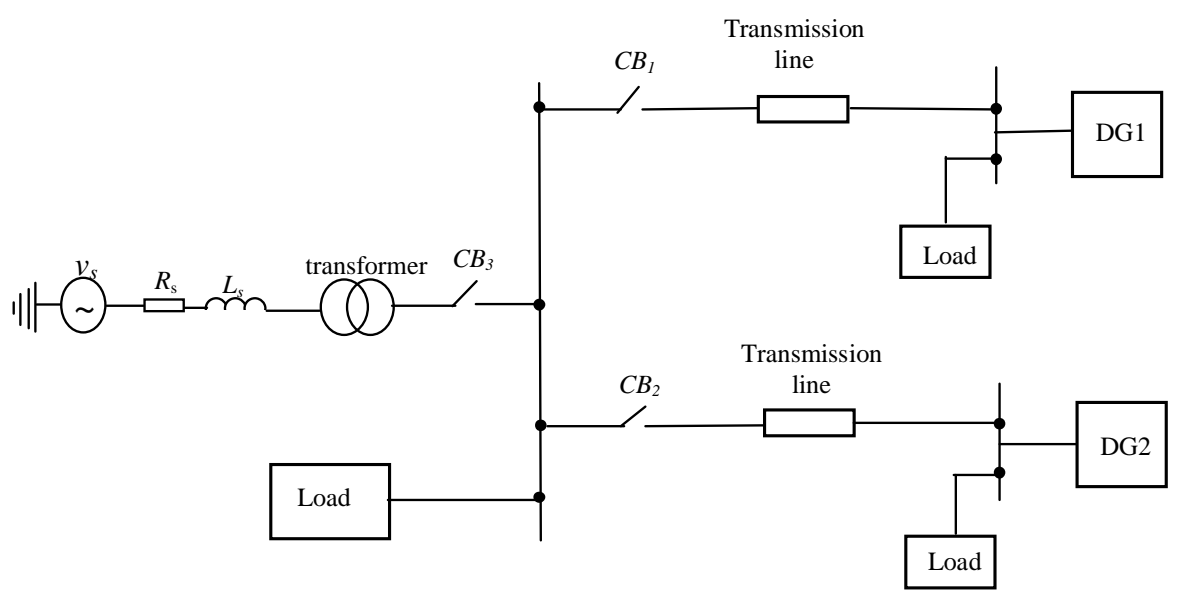

Figure 2.2: DGs in micro-grid connection

\subsection{Energy conversion}

The idea behind the energy conversion is to convert/harvest the maximum generated power from the renewable energy sources. For example, wind is used for wind turbine generation and solar radiation for solar energy production. Both of the sources come from the environment and cannot be controlled, where it will affect the output power. 
To help to control this output power, a power conditioning configuration can be used as shown in Figure 2.3; it uses wind speed as a source and consists of a synchronous generator, a diode rectifier and a dc-dc converter. For the wind turbine generator, it is most likely that the wind energy can be converted to electrical energy by using the Doubly Fed Induction Generator (DFIG), the synchronous generator and the permanent magnet synchronous generator that have been applied in $[13,14,15]$. The voltages generated from the generator are connected to the diode rectifier and the dc-dc converter in order to control the maximum power generated by the wind source where has been discussed in $[13,16,17,18,19,20]$.

This process described is known as Maximum Point Power Tracking (MPPT). For solar and fuel cell renewable energy sources, the electrical outputs are in dc signal and they are not constant due to the varying input source. To obtain the MPPT from this variable dc input power, the dc-dc converter can be used without using the rectifier $[20,21,22]$. As has been stated, the MPPT is related to the input source of the energy and it can be controlled when the control strategy is applied to the power conditioning side. For wind energy applications, the current at the dc-dc converter can been controlled according to the speed of the generator and it will determine the power generated from the generator. This concept, which has been applied to wind energy, can also been implemented in the ERLA configuration. It is by controlling the output current at the diode rectifier it will at the same time control the generated output power from the generator.

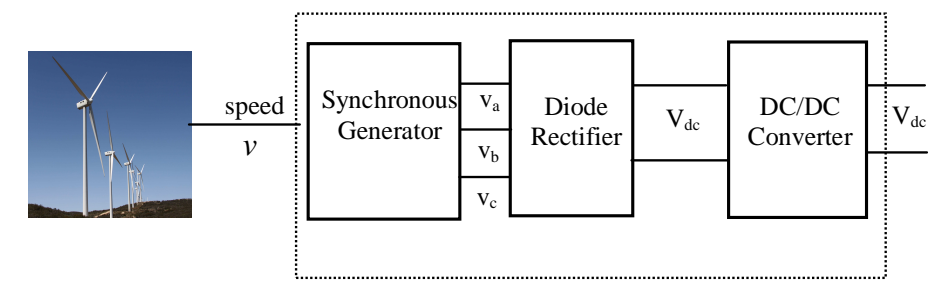

Figure 2.3: Topology of energy conversion in renewable energy source

\subsubsection{Linear synchronous machine in energy conversion}

For an landing aircraft application, the linear synchronous machine has gained interest due to the structure, the speed and the precision that can be achieved from it. Linear machines can be separated into two operations: motor operation or generator operation. In motor operation, the linear machine is used for the high precision location that 
is needed for the machine tool industry. For high speed systems, it is used in transportation such as the Maglev train application [23]. Linear generators are linear motion electromagnetic devices that transform an oscillatory motion of mechanical energy into electrical energy. In synchronous generator operation, the idea of regenerative braking that converts the energy from vehicle braking into electrical energy has been discussed $[7,24,25,26]$. For linear generator machines, regenerative braking is used mostly in ocean wave harvesting technology $[11,27,28,29,30,31]$ by converting the ocean wave energy into electrical energy. It is used in wave energy because the linear machine is capable of directly utilising the linear piston force without the need for an additional mechanical component, compared to a rotary device that needs a crankshaft mechanism to change the linear force to rotary torque and then transmit it to the generator.

The linear machine is where the stator and the rotor of the rotating machine have been cut and put flat on the surface, but the working principle remains the same as the synchronous generator. Figure 2.4 shows an example of a linear machine [32]. The advantages of the linear machine are that it drives the linear motion load without gears, screws or crankshafts, and it can also overcome the problems of stiffness, mass friction and backlash. It can also create a levitation force between the stator and the rotor due to a magnetic field which is used in high speed train application.

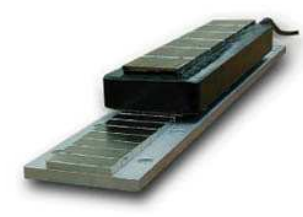

Figure 2.4: Example of a linear generator

The operation of the linear generator is based on mechanical motion; it generates a travelling magnetic field that is flowing in a horizontal direction which creates the voltage at the stator output. It also shows that the travelling magnetic field is equivalent to the velocity [33] of the rotor speed as given in equation 2.1:

$$
v=f_{e} \beta
$$

where $v$ is the velocity of the rotor, $f_{e}$ is the exciting frequency in hertz and $\beta$ is the winding wavelength. By expanding equation 2.1, the relationship between the synchronous generator with a linear generator can be given as: 


$$
\begin{aligned}
\theta & =\frac{2 \pi v t}{\beta} \\
& =\frac{2 \pi z}{\beta},
\end{aligned}
$$

where $\theta$ is the rotating mode in the synchronous generator while $z$ is the displacement for the linear generator. It can be summarised that for the linear generator, $z$ is the same as $\theta$ while the force $(F)$ is the same as the torque $(T)$ in the synchronous generator.

The concept of the linear generator is based on the travelling magnetic field [33, 34] which has gained interest for high speed applications. This travelling magnetic field is proportional along the speed, and it gives an indication that the linear generator is suitable for harvesting the energy during landing aircraft. For the ERLA structure that uses the linear generator structure, two techniques can be applied to the landing process as shown in Figures 2.5 and 2.6. Both figures illustrate the arrangement of the stator and the rotor during landing. For the first technique, the rotor is used as a park platform for the aircraft when it lands as shown in Figure 2.5. Here, the aircraft will move-up on the platform and then it will move together during landing. In the meantime the stator can be placed underneath the runway to capture this kinetic energy and change it to electrical energy.

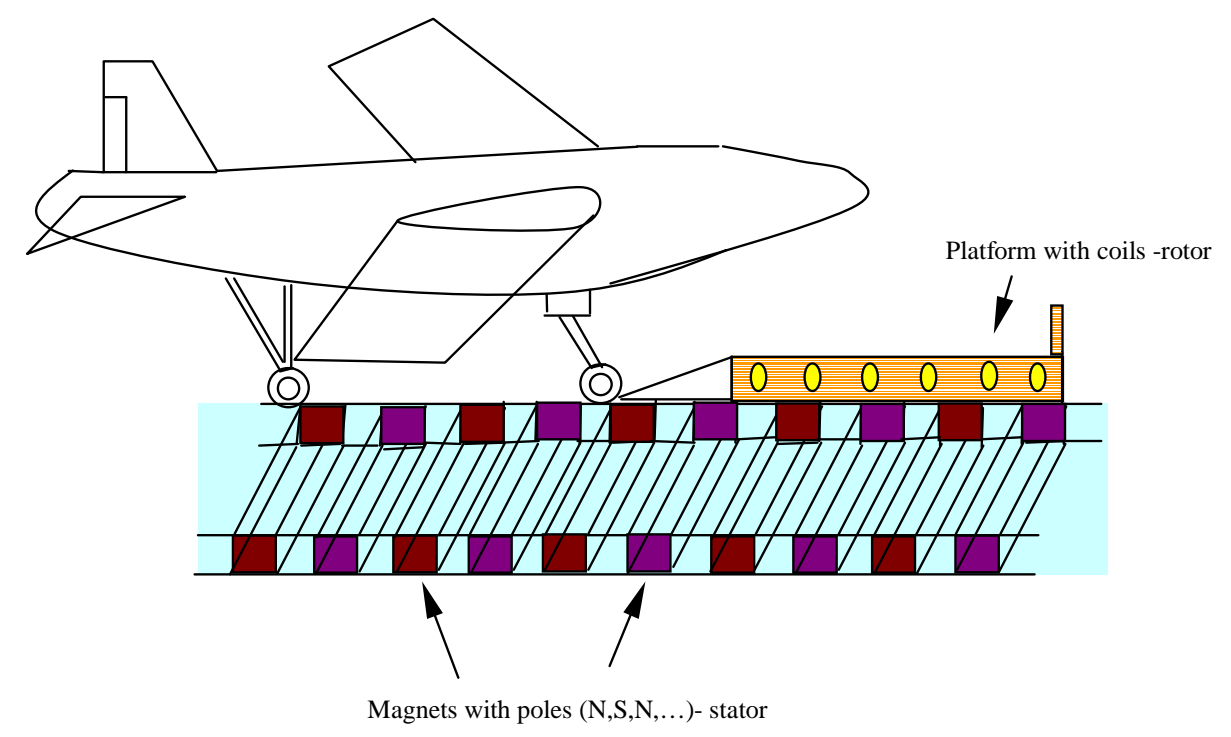

Figure 2.5: First technique of LG configuration in the ERLA application

The second technique is by pushing the rotor along the runway which is shown in Figure 2.6. This technique gives more advantage compared to the first technique in-terms of safety. This is because the landing speed can be controlled by the pilot. 
For example, when the condition of the runway is slippery or wet the pilot can release more breaking force in order to control the vehicle. For both techniques, the slot-less permanent magnet is used as a stator while the rotor consists of coil [9].

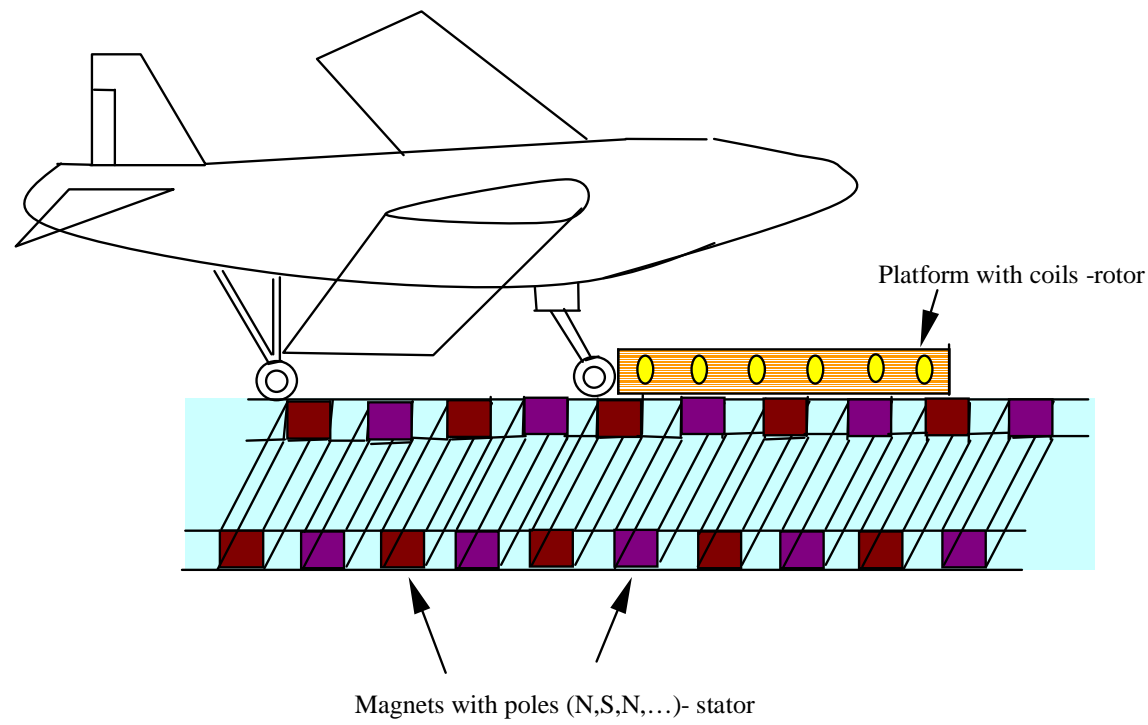

Figure 2.6: Second technique of LG configuration in the ERLA application

Many controllers can be applied to the linear generator depending on the function of the machine. The linear generator control in wave energy technology has been discussed here where it has the same function as the ERLA system. Currently, the control topologies for LG in wave technology are by using the power or force control. The power can be controlled if the current at the generator can be controlled [29]. This is done by ensuring that the current is the same as the electromagnetic force in magnitude and phase inside the LG. This will cause the power generated from the LG to be isolated. Other than the power control technique, the LG can also use the force control which is explained in $[27,35,36,37]$. This force is proportional to the velocity and displacement of the damping spring in wave energy applications. At the same time, this force is proportional to the current generated at the output of the LG. Because of this, by controlling the current, the force will be controlled, and the maximum power from the wave can be extracted [27]. This is when the current controller is used at the power conditioning converter to extract the maximum power from the LG. In [11], it shows that the LG is connected to the diode rectifier which will generate variable voltage and frequency at the outputs. Due to these variable outputs, the dc-dc converters are needed in order to give constant voltage and frequency outputs that can be used for energy storage and energy transfer sections. 
This power and force control can also be achieved by using the back to back converter, the rectifier, or the dc-dc converter [35, 38]. By using these converters, the control strategy can be made sensor-less to the speed. This is when the controller at the dc-dc converters do not use the information from the speed, but it is able to control the force at the LG. This will allow the control strategy to be applied at the power conditioning devices, rather than on the LG. In this case, it is capable of reducing the cost and number of controllers that will be used in the ERLA system application.

\subsubsection{DC-DC converter as power conditioning in energy conversion}

Wind, solar and fuel cell energy generate fluctuating output power at all times. Due to this, a converter is needed in order to control the output power flow to the storage element or to transmit it to the electrical grid network. In this case, the dc-dc converter can be used to control the power generated from the LG and at the same time enable the maximum power point tracking $[16,17,18,39,40]$. For wind energy source, the output voltage from the generator contains variable magnitude current, voltage and frequency. This also happens in the ERLA system, where the voltage and current outputs from the LG are reduced to zero which result from the reducing landing speed. This means that at the beginning, the power generated from the LG is at a maximum because this is the time when the maximum landing speed is applied to the LG. After that, it will start to reduce to zero. In this case, it causes the LG outputs to generate a wide range of input voltages and currents that need to be controlled by the dc-dc converter. Some common dc-dc converters that can be used in wide range input are the boost, buck-boost, SEPIC or Cuk [41] converters. Apart from these, several other topologies can be used which are listed below:

- the single switch or buck-boost converter shown in Figure 2.7a,

- the two switch, as shown in Figure 2.7b,

- the three level boost converter as in [42],

- the cascaded, interleaved and superimposed connection of the converter [43].

The single switch or boost converter is a conventional dc-dc converter that is used in power tracking for wind power or fuel cell power applications [17, 18, 44, 45, 46, 47]. In order to achieve the MPPT, the controller for this converter needs an algorithm to calculate the maximum power which responds to the suitable duty cycle for the converter. In this case, the power function, which consists of wind speed and the previous power, needs to be developed. This converter creates a high stress value on the inductor and the capacitor and it will increase the cost of the converter [43, 48]. 


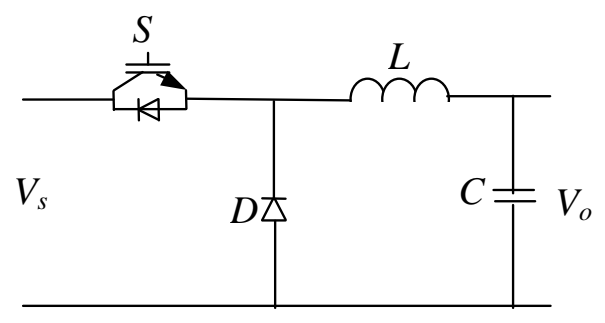

(a) single switch boost converter

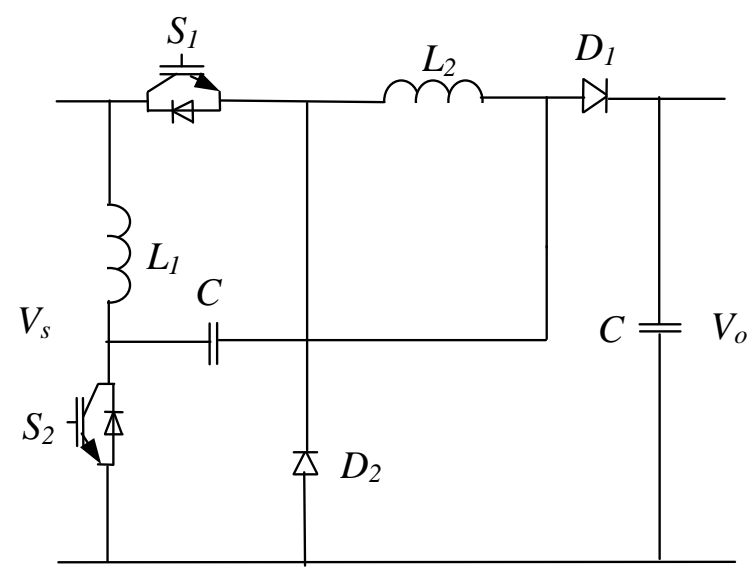

(b) two switches or three level

Figure 2.7: Example of dc-dc converters that can be applied in energy conversion

The two switches application is more appropriate because the stress of the inductor can be dissipated by reducing the size of the inductor and capacitor, compared to the single switch dc-dc converter [43, 41]. The cascaded buck-boost converter with two switches, known as the non-inverting buck-boost converter, has been used to regulate the variable input voltage and it has non polarity inversion at the output with regards to the input voltage $[42,49,50,51,52,53,54,55,56]$. This structure generates the direct path for the power flow from the input to the converter, and the losses at the inductor are at a minimum $[43,49]$. The cascaded converter is shown in Figure 2.8 where it will be the focused to the ERLA system.

For the cascaded buck-boost converter, two control strategies can be applied which are the current and voltage control. For the current control, several types can be used such as the sliding mode control with proportional-integral (PI) [52], the two independent controls applied in [48], the unified controller suggested in [57] and the back stepping control with PI control proposed in [58]. All of these use input feedback current. The voltage control can be achieved by separating the operation mode of the cascaded buck-boost converter, so that for half operation time it operates as a buck 


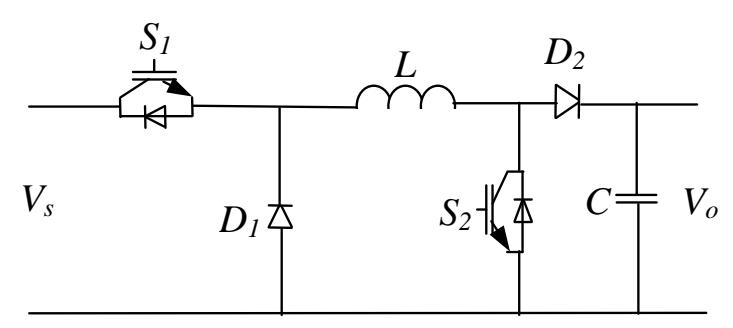

Figure 2.8: Circuit diagram of a cascaded buck-boost converter in energy conversion

converter and for the other half of the time as a boost converter by using logic sequences $[53,52]$. More complex controllers can be applied to improve the performance of the cascaded buck-boost converter by using the robust control technique in the average mode model explained in $[58,59,60]$.

Different structures of the buck-boost converter with more than two switches have been discussed in [43, 42, 50, 61]. For example, the three level boost converter gives less stress to the inductors compared to other converters. It is easy to model the controller that is based on the boost control structure, but the disadvantages are that it uses more inductors and switches. Due to the increasing number of switches, additional control loops need to be applied at the main controller.

In [43, 61], a more complex arrangement of the buck-boost converter has been used in order to minimise the stress of the inductor. First, the structure can be connected in an interleaved or superimposed connection, where these connections improve the stress and the conduction losses of the inductor compared to the others. These configurations will behave like a filter for the converter, and the controller needs to use a more complex controller which includes the three mode duty cycle. These converters create problems such as a higher harmonic current at the output [41, 48, 50].

As a solution, a cascaded buck-boost converter has been used in the ERLA application which is capable of giving a constant output from a wide range of input voltages and currents. It is also capable of changing the variable dc inputs to constant dc outputs in the continuous conduction mode at the inductor. By using the current controller combined with the logic sequence, the cascaded buck-boost converter is able to control the LG outputs in order to change the uncontrolled power output to control power output.

\subsection{Energy storage}

After the power from the energy conversion has been controlled, this huge amount of power at the beginning needs to be stored. Because of this, the energy storage part is essential. At the same time, the function of energy storage is to inject the energy 
to the load when the load requires more power than the source power can be provided from the energy conversion. It is also to ensure that a continuous power supply can be supplied to the load.

Most of the energy storage sources, it generates low voltage output compared to the input voltage at the energy transfer process which requires high voltage. Because of this, the boost converter is used to increase the voltage. For the energy storage element, it is combined with the bidirectional converter in order to have the function of absorbing and releasing the energy from the storage elements. Figure 2.9 shows the topology of the energy storage part in renewable energy source applications.

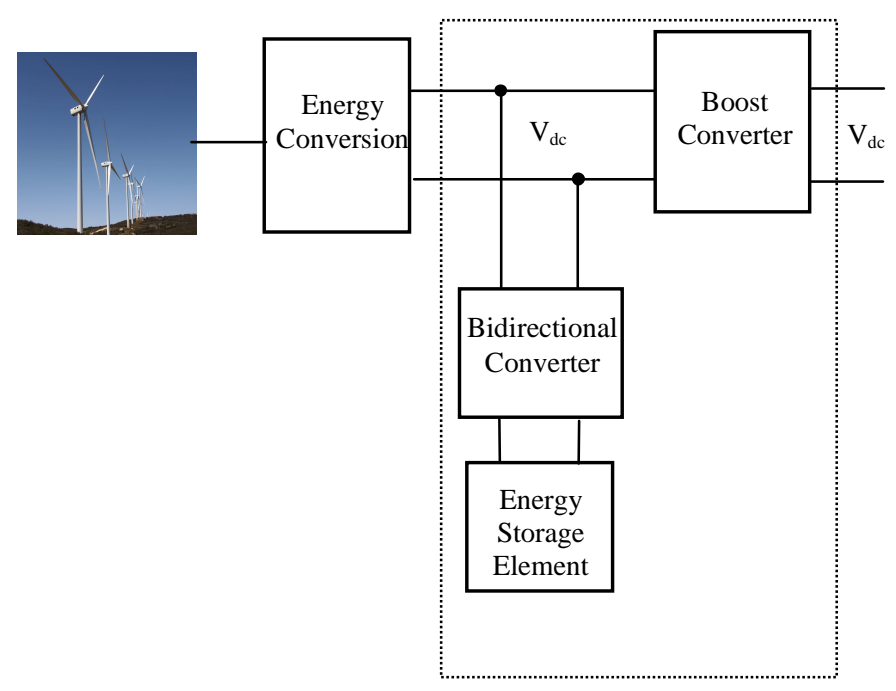

Figure 2.9: Topology of energy storage in renewable energy source applications

\subsubsection{Energy storage elements}

Generally, the functions of the energy storage elements in renewable energy, hybrid vehicles or uninterrupted power supplies are to provide power during transient, inject the power when the main source has a low power output such as in renewable energy sources, and to absorb the energy during vehicles braking [62,63,64]. Another function is as a temporary storage (short term) [65] or a transit place before it can be transferred to the load. For example, energy storage elements such as the ultracapacitor, battery, flywheel etc can be used as storage devices by connecting them to the dc-link $\left(V_{d c}\right)[44,66,67,68]$. For renewable energy source applications, the battery and the ultracapacitor can be used as storage elements to improve the reliability and the stability of the grid connection [44, 62, 63, 69, 70, 71, 72]. 
Due to its fast power regulation, the ultracapacitor is more suitable for use in landing aircraft. This is because it can respond to high power density, has a high charging speed, a longer life time and a high energy density output [64, 70, 73, 74, 75, 76]. The ultracapacitor stores the energy by separating the positive and negative charge, and it has a fast discharging rate due to the high rated current [75]. By placing the dc converter between the ultracapacitor and the dc-link, the direction of the current can be changed and at the same time it can control the voltage at the dc-link. The ultracapacitor can be modelled by the internal capacitance $(C)$ and with the series resistor $\left(R_{e s r}\right)$. This combination will give a more accurate expression for the ultracapacitor as given by $[70,77]$ :

$$
Z(s)=R_{e s r}+\frac{1}{C(s)}
$$

\subsubsection{Energy storage with bidirectional converter}

A bidirectional converter has the ability to allow two directions of power flow, either flowing from the energy storage elements to the dc-link voltage or vice versa. Most of this converter has been applied at the energy storage element in renewable energy applications and in uninterrupted power supplies [57, 63, 66, 78]. The bidirectional converter structure resembles the half bridge circuit which consists of only one inductor in order to reduce the current stress and also to give high efficiency. The bidirectional converter with the energy storage element is connected in parallel to the dc-link voltage in order to regulate the dc-link voltage, and to discharge the stored energy [76].

This bidirectional converter can be controlled depending on the type of energy storage element that will be used. If the battery is used, this converter is used to control the state of charge with a state control for optimum charging, while when the ultracapacitor is used, it is to regulate the dc-link voltage $[40,71,79]$. Other than these it can also use the inner loop current control combined with the other voltage loop that been proposed in $[67,72,76]$, the finite state machine in [64], or the dynamic evolution control in which the switching angle was calculated by [69]; these are able to control the power flows from the bidirectional converter. In [80], it has been suggested to use the state of charge for the ultracapacitor for the power flow process. A more advanced control strategy that includes 3 modes of operation for the bidirectional converter which are buck, boost and shutdown can be applied combined with a power management application, in order to have the current flow between the energy storage and the dc-link [66, 81].

More complex methods can be applied to control the bidirectional converter. One of the solutions is by changing the nonlinear equation that is caused by the switches to a linear equation by using the average control technique. This is done by integrating 
the switching cycle in order to produce a constant signal at the output $[51,82]$, which will be used to determine the controller compensator.

\subsubsection{Boost converter}

The boost converter is used to increase the dc-link voltage to the desired output voltage in order to be used by the energy transfer. This is to ensure that the input voltage at the inverter is capable of generating 3-phase voltage when it connects to the existing electrical network or to the load. The boost converter has been used widely in renewable energy structures $[62,83]$ because is easier to build and to control. The boost converter consists of one inductor and one switch which will respond to the controller target.

As is known, the boost converter is a nonlinear element which creates a nonlinear equation in which only the nonlinear control can be used. This nonlinear condition can be solved by using a controller that has a wide bandwidth response such as the PI controller [84], or the proportional-integral derivative (PID) controller which uses a genetic algorithm in order to determine the duty cycle gain for the controller [85], or by using the sliding mode controller (SMC) that has been proposed in $[86,87,88]$.

\subsection{Energy transfer}

All the power from the main source and the storage element need to be transferred to the grid. This part consists of the inverter, the filter and the grid source as shown in Figure 2.10. The inverter can be driven by power electronics devices such as GTOs, MOSFETs, FETs and IGBTs. The choice of power electronics devices is based on the rated output power of the inverter. Due to the switching effect of the power electronics devices, the inverter will inject a high current harmonic to the grid, and increase the total harmonic distortion to the grid current. In order to solve this problem, a filter can be connected between the inverter and the grid. The inverter also functions as the synchronisation component between the inverter voltage and the grid voltage. Synchronisation must happen between the inverter and the grid in order to transfer the power to the grid without causing any damage to it.

\subsubsection{Filter component at the energy transfer part}

To improve the injected current and to transfer clean sinusoidal current to the grid, three types of filter such as L, LC or LCL can be used. The L filter consists only the inductor; here the inductor size is bigger thus reducing the dynamic of the system. It also creates a longer response time for the current [20]. In order to improve this, the capacitor $\mathrm{C}$ is introduced, and is known as the LC filter. In this case, the inductance 


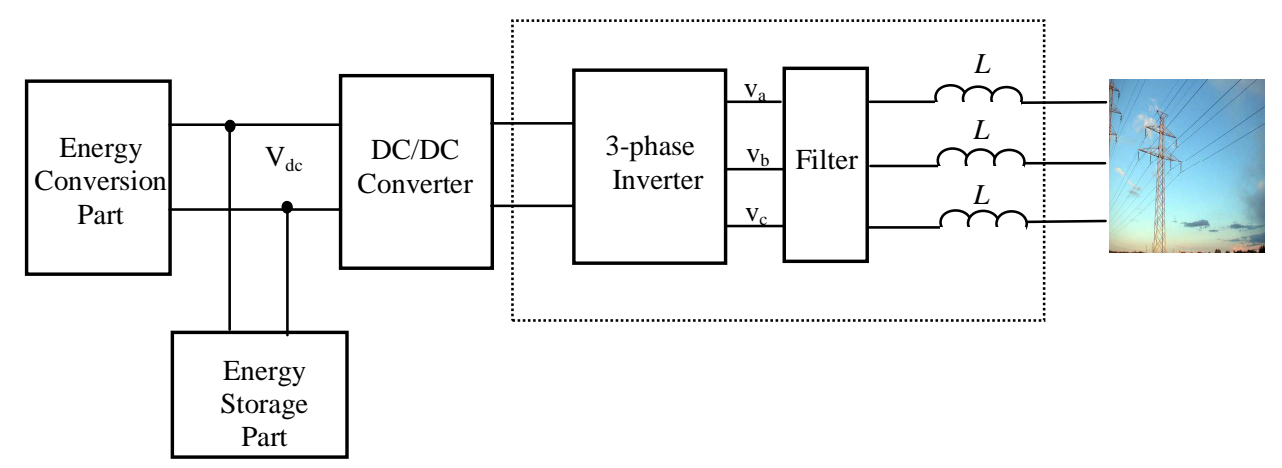

Figure 2.10: Topology of energy transfer process for renewable energy source

value can be reduced by selecting a very large capacitance which will create an inrush current; this high capacitance is not suitable for current injection [89]. Due to these disadvantages of both filters, most researchers have used the LCL filter model. This is also known as the electromagnetic interference (EMI) filter and is used at the inverter to prevent high harmonics at the switching frequency. It will give low ripple current, prevents inrush current, and leads to small values for the inductor and the capacitor which are suitable to be used for the grid connection [20, 89, 90, 91, 92, 93].

\subsubsection{Synchronisation of the inverter with the grid source}

Synchronisation of the DG structure is important in order to ensure that the power can flow from the DG to the electric grid network. For normal synchronous generator operation, the synchronisation can be established when the frequency of the rotating flux in the synchronous generator is the same as the grid frequency. When a fault happens, both frequencies are not the same, and the generator will be separated from the grid. It takes a longer time to synchronise back. The DG synchronisation is better than the generator because it is not related to the rotating flux angle, but the inverter needs the information about the amplitude, the phase angle and the frequency of the grid source which can be collected using a proper synchronisation technique before it can be synchronised.

The most common or most used synchronisation method is the phase-locked loop (PLL) applied in [94, 95, 96, 97]. The operation of the PLL is when the feedback response of the voltage controller oscillator is used to lock the phase of the grid voltage. Another technique is the sinusoidal tracking algorithm (STA), which is able to detect the frequency changes in the grid faster then the PLL and with a high setting time [98]. This STA is also able to lock the phase and the frequency if the grid voltage is not 
balanced. A new kind of synchronisation, known as the synchronverters structure which does not use the PLL, has been implemented in [99]. It makes the inverter behave like a synchronous generator with frequency droop control for synchronisation of the inverter and the grid.

\subsubsection{Power flow between the inverter and the grid source}

Power flow refers to the power interchange between the main source and the grid network. In the DG unit, the renewable energy is the main power source while the inverter is a drive structure to transfer the energy to the grid. This power flow can be achieved by using a proper control application on the inverter. The current or voltage control strategy can be used in order to transmit the power from the inverter to the grid. Here, most researchers have used the current control application [94, 100, 101]. By adding the power control to be the outer loop control while the current control is the inner loop, the amount of power flow that is related to the injected current can be established $[17,97,93]$. The equations that relate the power with the voltage and current at the inverter are given as:

$$
\begin{aligned}
P & =v_{a} i_{a}+v_{b} i_{b}+v_{c} i_{c} \\
Q & =\sqrt{3}\left[\left(v_{a}-v_{b}\right) i_{a}+\left(v_{b}-v_{c}\right) i_{b}+\left(v_{c}-v_{a}\right) i_{c}\right] .
\end{aligned}
$$

where $P$ is the real power, $Q$ is the reactive power, $v_{a}, v_{b}, v_{c}$ are the line to line voltage and $i_{a}, i_{b}, i_{c}$ are the inverter line currents.

From equations 2.3 and 2.4, the injected real and reactive power are easier to control in the inverter compared to the synchronous generator, where the current at the inverter terminal is used as the feedback control and the PLL block is used for the synchronisation.

\subsubsection{Control scheme for inverter-side converter}

Basically, the structure of the grid side inverter is monopolised by the voltage source inverter (VSI) due to the high power rating, and the time response is faster than the current source inverter (CSI). As is known, the function of the inverter is to inject the current to the grid by using the current control, in order to have a power flow which is important for renewable energy generation. Meanwhile, the voltage control is used to improve the power quality at the terminal voltage grid caused by voltage sag, voltage swell etc. 


\subsubsection{Current control for the inverter}

Due to the maturity of this control structure, most researchers use this strategy for the grid connected system. Figure 2.11 shows the current control topologies for the inverter based on the natural frame control.

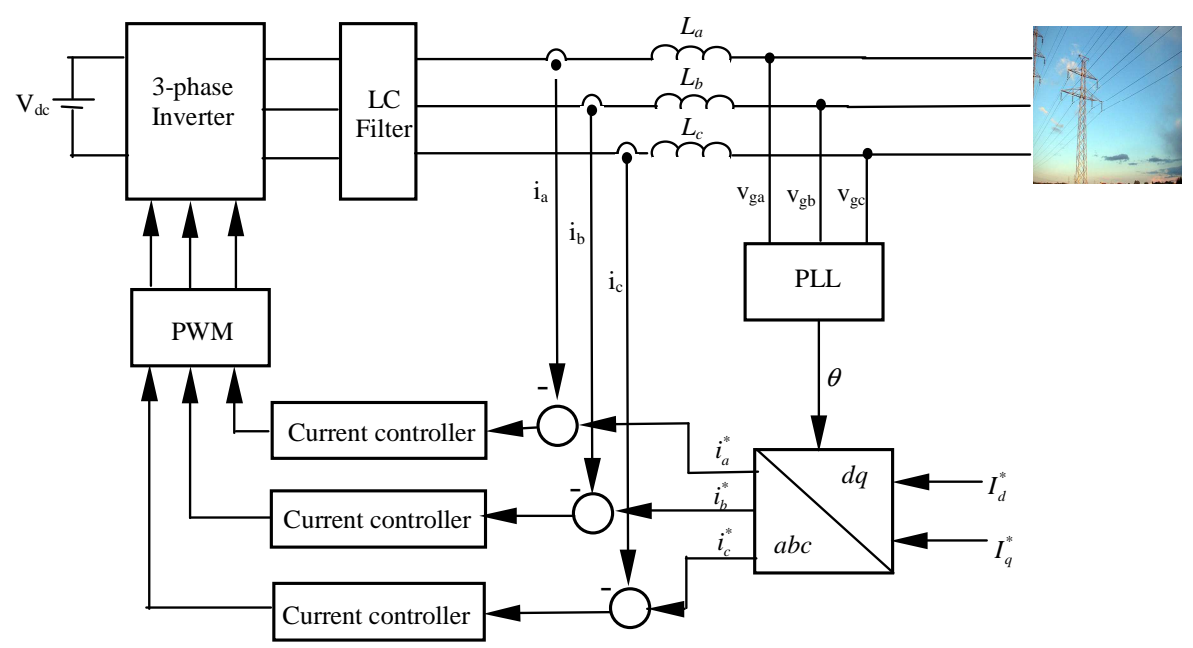

Figure 2.11: The block diagram of current control for energy transfer process in natural frame $(a b c)$

Figure 2.11 shows that, each phase has its own current control to create a more dynamic response [20] compared to use only one current controller. Here, it gives a high accuracy control for instantaneous current, a peak current protection, an overload rejection and has a very good dynamic [20, 94, 102]. These advantages ensure that the tracking error between the reference currents and the inverter currents can be kept to a minimum in order to inject less Total Harmonic Distortion (THD) to the grid. The $\mathrm{P}+$ Resonant controller with harmonic compensation is also able to inject less THD current to the grid if it has been implemented at the inverter control. The dead-beat controller, the hysteresis and the PI controllers can also be used for current control [92, 97, 103] for the current injection from the inverter.

All of these controllers are known as the classical control approach. Recently, more robust controllers have been adopted for the inverter. The $H^{\infty}$ control theory has been put into practice in order to control the injected current by the inverter to the grid $[100,101,102]$. This controller is able to minimise the tracking error and at the same time it can operate during the unbalanced condition, or when a nonlinear load is connected to the DG. Meanwhile, the existence of the LC filter shown in Figure 2.11 requires a more complex current control strategy in order to maintain the stability [92]. Due to this, the current controller design must include the LC filter and the grid 
inductance $\left(L_{a}, L_{b}, L_{c}\right)$ parameters for the controller calculation which can be achieved by using the robust control strategy.

Figure 2.11 shows the reference currents $\left(I_{q}^{*}\right.$ and $\left.I_{d}^{*}\right)$ coming from the outer power control technique. The $a b c-d q$ transformation is used in order to change the reference currents from $I_{q}^{*}$ and $I_{d}^{*}$ format to three phase format $\left(i_{a}^{*}, i_{b}^{*}\right.$ and $\left.i_{c}^{*}\right)$. The error signal between the reference and feedback currents will be fed into the current controller block in order to generate the required pulse width modulation (PWM) signal to the inverter for the power electronics devices.

\subsubsection{Power control technique at the inverter}

Power control is used at the inverter in order to generate the reference currents for the current control which are proportional to the required power. There are two types of power control that can be used for the inverter, the first is the Direct Power Control (DPC) and the second one is the active and reactive power control.

The DPC strategy has been used in [13, 98, 104, 105]. The DPC is based on the Direct Torque Control (DTC) which has been used in controlling the motor torque regarding to the rotating flux. For the DPC control, the switching signals that are used for the inverter switches are generated from the switching table which is based on the error between the reference and the measured real and reactive power [13]. The advantage of using DPC is that the inner current loop and PWM switching model are not needed. It is because the PWM switching is based on the look-up table that is based on the hysteresis control [98, 106].

For this control technique, the reference real power $P^{*}$ is generated from the $\mathrm{dc}$ voltage controller, and the reference reactive power $Q^{*}$ is set to zero for the unity power factor. The unity power factor means that the voltage and current at the grid terminal are in the same phase angle. The generated signal is based on three conditions which are the $P, Q$ and the flux vector position $\checkmark$ of the power. The generated signal can be expressed as:

$$
\begin{aligned}
& S_{p}= \begin{cases}1 & P<P^{*} \\
0 & P>P^{*}\end{cases} \\
& S_{q}= \begin{cases}1 & Q<Q^{*} \\
0 & Q>Q^{*}\end{cases}
\end{aligned}
$$

By addressing the $\checkmark$ with the $S_{p}$ and $S_{q}$ value, the appropriate voltage vector can be selected from the look-up table. Figure 2.12 shows the convectional DPC structure.

For real and reactive power control, it is based on the $d q-a b c$ transformation and the feed-forward of the inverter dc-input voltage. This transformation guarantees the fast 


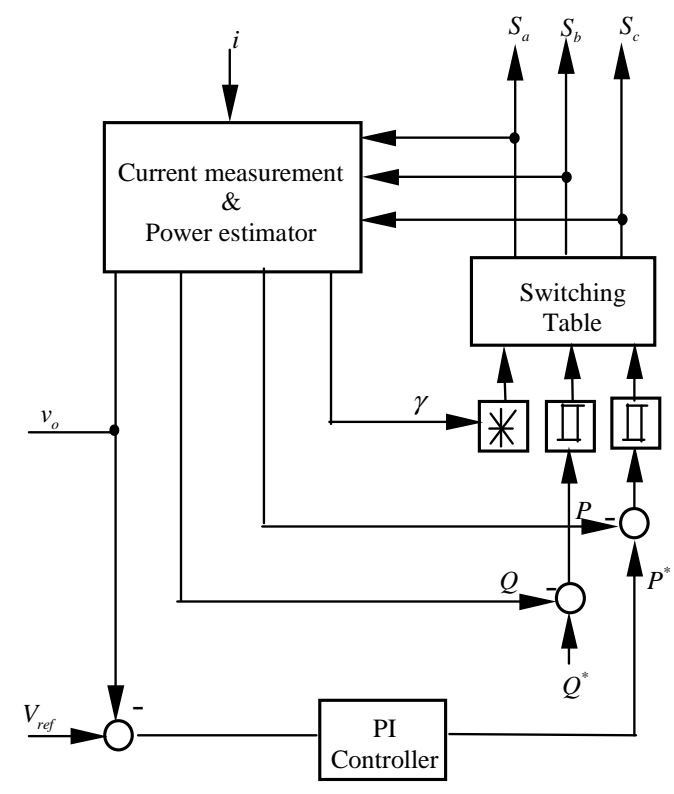

Figure 2.12: The block diagram of DPC control strategy

transient response and gives high static performance with the inner current controller for the inverter which has been applied in [38, 97, 107]. The real power control and the dc-voltage control will correspond to the $I_{d}^{*}$, while the reactive power will correspond to $I_{q}^{*}$ as shown in Figure 2.13. This power control is known as voltage oriented control (VOC), where the reference currents $\left(I_{d}^{*}, I_{q}^{*}\right)$ are the output from the power and reactive power calculation in $[99,108]$. Figure 2.13 shows the complete control strategies applied to the energy transfer.

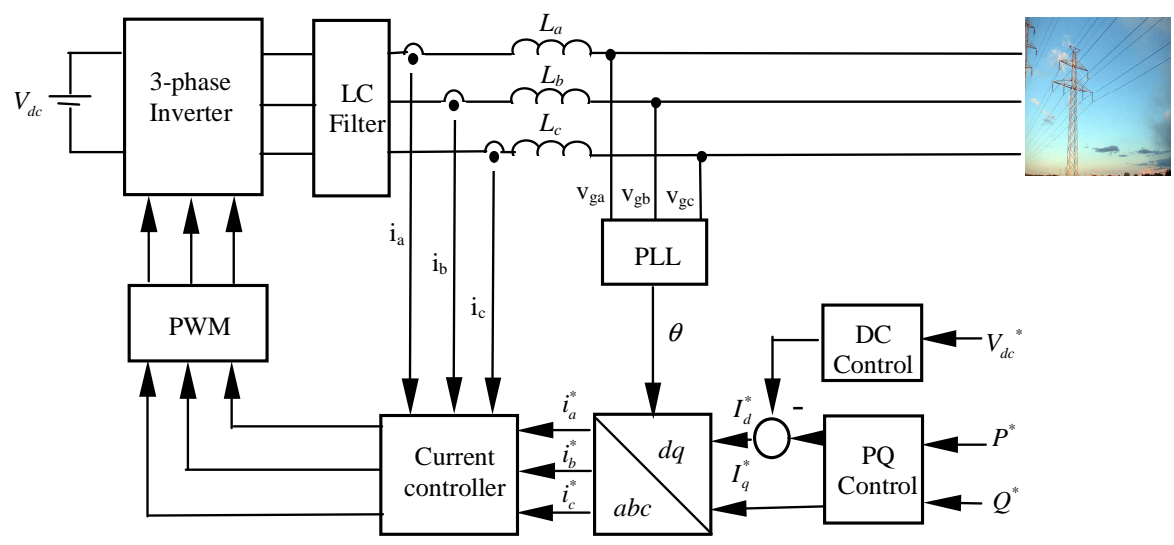

Figure 2.13: The block diagram of power and current control in the energy transfer process 


\section{$2.6 \quad H^{\infty}$ control theory}

The $H^{\infty}$ control theory is used in controller design to minimise the tracking error, to improve the stability, to give a robustness effect to the plant $[61,105,109]$ and to guarantee the performance in the worst case disturbance $[110,111]$. Recently this control theory has been applied to the dc-dc converters [21, 112, 113], grid connected inverters [100, 101], Flexible AC Transmission System (FACTS) devices [114] and Permanent Magnet Linear Synchronous motor (PMLSM) for position tracking [111].

The name $H^{\infty}$ comes from the name of the mathematical space over which the optimisation takes place (Hardy place). As is known, this control theory is in the robust control group, where the controller in this group includes the load variation, the perturbations and the disturbance effect of the plant in order to calculate the compensator controller value. The standard configuration for the $H^{\infty}$ optimal control application to the plant is shown in Figure 2.14. $K$ is the stabilising compensator that will be calculated using the $H^{\infty}$ control theory while $G$ is the plant that needs to be controlled.

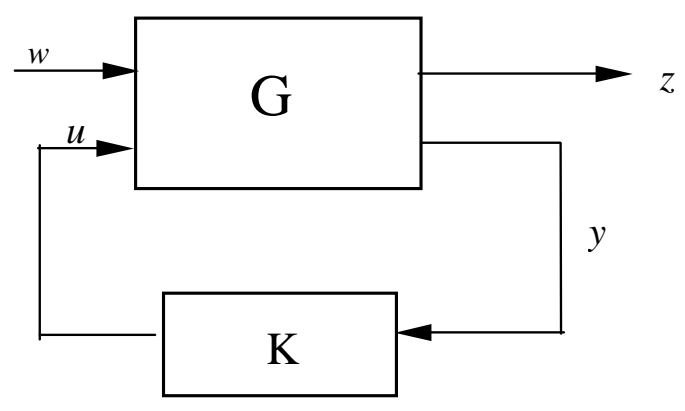

Figure 2.14: The standard $H^{\infty}$ optimal control formulation

There are four signals involved in the $H^{\infty}$ model, which are the $w, u, z$ and $y$. The $w$ signal represents external disturbance inputs and $z$ is the regulated performance outputs, which include the weighting functions that are used to improve the robustness and stability of the controller [21, 115]. In the mean time, $u$ is known as the control input for the system, while $y$ is the target output from the plant. The $G$ can be any equivalent electrical circuit, and it can be written in state-space equations $[100,101]$ or in transfer function equations $[21,116]$. For example, the state-space equation for the $G$ can be written as:

$$
\begin{aligned}
\dot{x} & =A x+B_{1} w+B_{2} u \\
z & =C_{1} x+D_{11} w+D_{12} u \\
y & =C_{2} x+D_{21} w+D_{22} u
\end{aligned}
$$


where $A, B_{1}, B_{2}, C_{1}, C_{2}, D_{11}, D_{12}, D_{21}$ and $D_{22}$ are in matrix format in order to generate the extended plant for the $H^{\infty}$ control calculation that also includes the inputs to the plant which are $w$ and $u$. The $x$ is the state variables of the plant. Equations 2.7, 2.8 and 2.9 can be written in matrix notation as:

$$
G=\left[\begin{array}{c|cc}
A & B_{1} & B_{2} \\
\hline C_{1} & D_{12} & D_{12} \\
C_{2} & D_{21} & D_{22}
\end{array}\right] .
$$

Equation 2.10 is the matrix plant, and the extended plant $G$ (new) with the weighting functions $(W)$ is given by:

$$
\begin{aligned}
G(\text { new }) & =\left[\begin{array}{c|cc}
A & B_{1} & B_{2} \\
\hline C_{1} & D_{12} & D_{12} \\
C_{2} & D_{21} & D_{22}
\end{array}\right] \times W \\
& =\left[\begin{array}{ll}
G_{11} & G_{12} \\
G_{21} & G_{22}
\end{array}\right] .
\end{aligned}
$$

From equation 2.11, the value of the compensator can be calculated by using the available functions based on the Ricatti Equation in order to calculate the optimal value of the compensator. The functions of hinfsyn or mixsyn can be used to calculate it in the robust control toolbox which is available in the MATLAB [114, 117, 118].

\subsection{1 $H^{\infty}$ control application in dc-dc converters}

The application of $H^{\infty}$ control theory in dc-dc converters has been widely used recently, for example it is used in the buck-boost converter with single switch [109] and in the boost converter [21, 113]. The reason why the $H^{\infty}$ has been used with dc-dc converters is to regulate the output by minimising the tracking error [21, 112, 113]. This controller has been proven to minimise the tracking error and at the same time it gives low output impedance in order to reduce the output ripple [21, 113]. These advantages are achievable because the design compensator includes the plant components in order to calculate the compensator by using the $H^{\infty}$ control theory.

\subsection{2 $H^{\infty}$ control application in the inverter-grid connection}

$H^{\infty}$ control theory has recently gained more interest with researchers for application in the 3-phase inverter. This is because it reduces the tracking error and gives a high dynamic response at the outputs. This occurs because the calculation of the controller compensator value has included the filter components and the disturbance effect that appeared at the inverter plant. The disturbances in the inverter are given by target 
output such as the reference value and also the grid voltage. Other than this, the $H^{\infty}$ control is a method that is able to respond to any changes in the perturbations and the variations at the inverter caused by load changing.

Many researchers have applied the $H^{\infty}$ control theory to the inverter. For example, this can be done by combining the repetitive control with the current controller, or the voltage controller structure, or the cascaded voltage and current which have been carefully explained $[89,101,119]$. The $H^{\infty}$ control theory has also been applied to FACTS devices, such as Dynamic Voltage Restore (DVR), in order to solve the voltage sag and unbalanced voltage in the grid system [114]. It has also been applied to the Unified Power Quality Conditioner (UPQC) that is used to solve all the power quality problems in the grid system [120].

\subsubsection{System stability}

For the compensator value $K$ which has been generated from the $H^{\infty}$ control theory, the stability of the compensator with the plant need to be determined in order to minimise the tracking error and to meet all the uncertain conditions [121, 122]. This stability can be calculated from the $H^{\infty}$ optimal problem when the closed loop feedback from the $z$ to the $w$ which makes the system insensitive to the external disturbance is determined $[116,119]$. Figure 2.15 shows the model to calculate the stability value. The transfer function for the stability can be written as:

$$
T_{z w_{1}}=\frac{G_{21} K}{1+G_{21} K} \times W_{1} .
$$

From equation 2.12 the condition that needs to be met in order to declare it stable is when $[100,101]$ :

$$
\left\|T_{z w_{1}}\right\|<1
$$

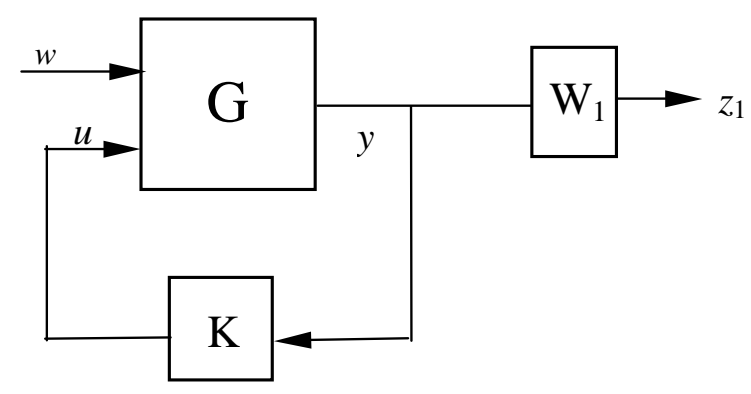

Figure 2.15: The stability block for $\left\|T_{y u}\right\|$ in $H^{\infty}$ control 
The stability of the compensator can also be calculated between the input $u$ to the output $y$ or $\left\|T_{y u}\right\|$ as shown in Figure 2.15. This $T_{y u}$ transfer function is known as closed loop feedback loop control. Equation 2.14 shows the transfer function of the feedback controller with the plant and the weighting functions. The control feedback law can be developed by referring to Figure 2.14 which is based on $[116,123]$. The stability equation can be written as:

$$
T_{y u}=G_{11}(s)+G_{12}(s)\left[I-K(s) G_{22}(s)\right]^{-1} K(s) G_{21}(s),
$$

where the $G_{11}, G_{12}, G_{21}, G_{22}$ are given from equation 2.11 and $I$ is the identity matrix. This technique can be used if the transfer function equation is used to model the plant, which is more suitable for the dc-dc converters. After the $T_{y u}$ of the feedback has been determined by using equation 2.14 , the magnitude stability condition can be determined by referring to the equation:

$$
\left\|T_{y u}\right\| \leq \gamma
$$

where $\gamma$ is gamma in the pre-specified attenuation level for the sub-optimal control which needs to ensure that $\left\|T_{y u}(s)\right\|_{\infty}=\max _{\omega} \bar{\sigma}\left(T_{y u}(j \omega)\right) \leq \gamma$.

The $\gamma$ and the $T_{y u}$ can be found using the hinfsyn function in the MATLAB program. To calculate the magnitude value of equations 2.12 and 2.14, the normhinf function, available in the Robust Control Toolbox in the MATLAB software, can be used to calculate the stability value. If the calculated $K$ value satisfies the condition given in equations 2.13 and 2.15, it means that the $K$ is stable and it can be used to minimise the tracking error under the disturbance and components effect in the plant.

\subsection{ERLA configuration in the renewable energy applica- tion}

Figure 2.16 shows the arrangement for the complete process of Energy Recovery From Landing Aircraft (ERLA). The configuration of ERLA network is based on the DG structure that has been used in renewable energy generation. At the end of the processes, electrical output can be generated, and it can be connected to the distribution grid network. The linear generator, the uncontrolled diode rectifier and the dc-dc converter model are used for the energy conversion, which will change the kinetic energy to electrical energy by controlling the current flows at the buck-boost converter. Controlling the buck-boost converter will at the same time control the LG outputs that are more related to the passenger comfort in the aircraft; one of them is the braking force. The LG has been chosen because the concept of the travelling magnetic flux, which flows in the horizontal direction, is equivalent to the speed vector during landing. The 


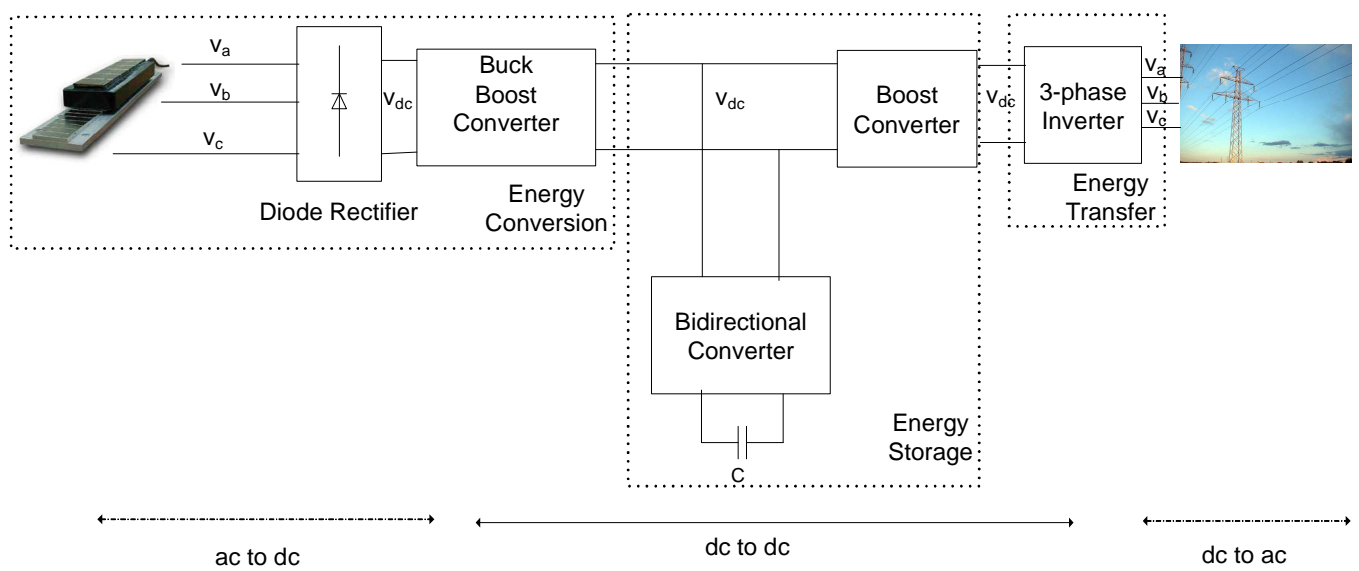

Figure 2.16: Overview of an energy recovery system for landing aircraft

diode rectifier is used to change the ac input from the linear generator to dc output in order to minimise the complexity of the system.

It is most likely that, energy storage is used to absorb/release the energy at the dc-link. A voltage control strategy is used to control the voltage at the dc-link between the energy storage and the energy conversion. It also allows the power flow to flow in both directions. It consists of an energy storage element, a bidirectional converter and a boost converter. The boost converter is used to increase the dc bus voltage $V_{d c}$ to a suitable value which can be used for the energy transfer application.

Energy transfer is a combination of a 3-phase inverter, the filter and the grid in order to transfer the power from the energy conversion and energy storage to the electrical network. Here, the current control and the real and reactive power control have been used in order to control the injected power and current to the load.

\subsection{Concluding remarks}

The energy from landing aircraft is a potential alternative energy source which can generate high power compared to the single wind turbine and at the same time is easier to control the power. In dealing with this energy, the energy processes can be divided into three categories which are the energy conversion, the energy storage and the energy transfer using the distribution generation network as the reference. For the energy conversion, the LG, the diode rectifier and the buck-boost converter are used to convert the kinetic energy to electrical energy. The LG has been selected because of its capability for responding to the speed which is proportional to the magnetic travelling flux which is flowing inside the LG. As a consequence, the electrical outputs from the LG 
contained variable magnitude and frequency. Due to this, the rectifier is used to change this variable output to dc signal output for the current and voltage. Here, the buckboost converter is used to control the input current in order to give constant magnitude output at the LG. The energy storage, consisting of the energy storage element, the bidirectional converter and the boost converter are used as a temporary storage place before the energy can be released to the load. For the energy transfer, the function of the filter, the synchronisation and the power flow need to be used in order to give the power transfer between the energy source and the energy storage which responds to the energy required at the load. Finally, the concepts of $H^{\infty}$ control theory have been applied to the dc converter and ac converter in order to implement this control strategy in the ERLA system. 


\section{Chapter 3}

\section{Energy conversion:Modelling of the linear generator}

This chapter explains the LG model in the energy conversion model. It is used to convert kinetic energy to electrical energy. The LG has been modelled in the 3-phase model and in the $d-q$ transformation model. The LG is suitable, compared to other types of the generators because of its mechanical structure, which is in flat mode for the stator and rotor, and it also responds to the speed that induces the travelling magnetic flux. Both of these models have been designed and used in order to see the outputs generated from the linear generator, which are the mechanical outputs such as the deceleration speed, the force and the distance travelled by the LG in the ERLA application. In the meantime, the electrical outputs have been measured at the diode rectifier. All the models have been designed in MATLAB Simulink environment, which are based on the equations generated from the LG model.

\subsection{Potential kinetic energy generated during normal land- ing}

During landing, a huge amount of kinetic energy can be collected due to the huge mass of the aircraft. This potential energy also depends to the losses of the braking force, braking tyres and the impedance inside the LG generator. Assume, for a normal aircraft such as the Boeing 747 with a mass of $m=40$ ton and the landing speed $(v)$ of $150 \mathrm{mph}$, the kinetic energy stored in this aircraft is:

$$
\begin{aligned}
E & =\frac{1}{2} m v^{2} \\
& =\frac{1}{2} \times 40 \times 10^{3} \times(0.4470 \times 150)^{2} \\
& =90 \mathrm{MJ}
\end{aligned}
$$


From this amount of kinetic energy, the power which can be generated is equivalent to $25 \mathrm{kWh}$. This amount of power can supply 2 days electricity to a three bedroom house based on the study conducted by [124]. As a result, there is a possibility to harvest the energy from the landing process. Before this potential power can be used, several power conditioning devices will be put in place in order to control, to store and to transfer this energy.

This work, has not used the actual value of the aircraft due to the limitation of the simulation storage memory. In order to avoid this problem, a small value of this mass and speed are applied in the simulation. In order to understand the power flow, the flywheel has been build in the lab in order to behave like the generates energy from the landing. The mass value of $m=100 \mathrm{~kg}$ and the speed $v$ of about $10 \mathrm{~m} / \mathrm{s}$ for the flywheel are used to equation 3.1 where it produces about 5000J which is equivalent to $1.39 \times 10^{-3} \mathrm{kWh}$. This energy can produce about $1250 \mathrm{~W}$ average power for about $4 s$. These parameters are used in order to determine the rest of electric components that is used in ERLA where it been calculated using the maximum generated power. This is the reason why the parameters have been selected to this work. To obtain this power, the LG and the diode rectifier are used and without any control strategy applied to them. Figure 3.1 shows the arrangement of the first part of the energy conversion during the landing.

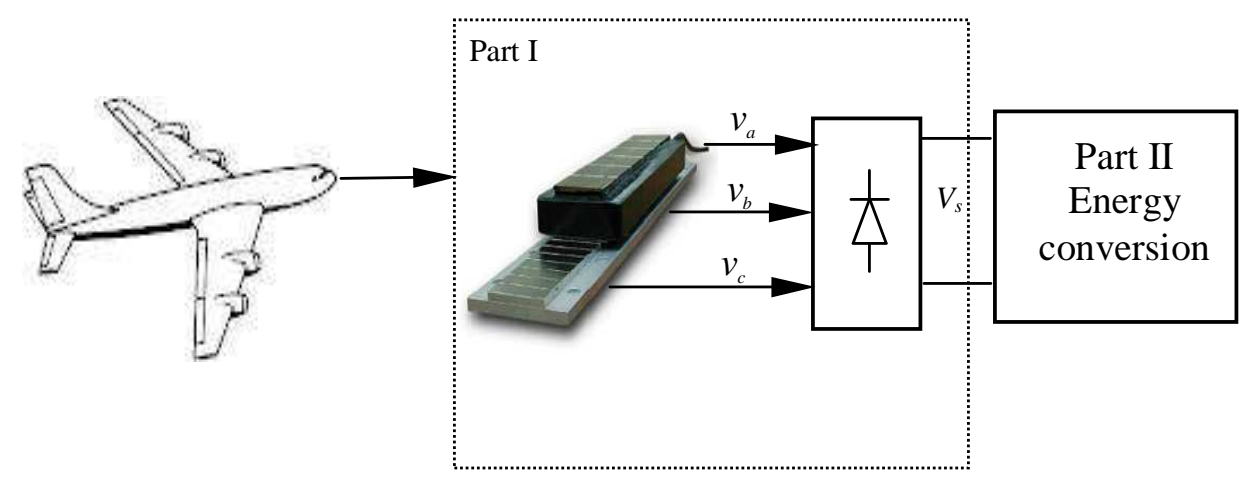

Figure 3.1: The block diagram of the energy conversion part

\subsection{Overview of a 3-phase synchronous generator}

Before the LG can be modelled, the basic operation of the synchronous generator needs to be understood. The synchronous generator is used to convert mechanical energy such 
as wind energy, water energy and thermal energy such as heat, into electrical energy by driving the rotor. The synchronous generator consists of two parts which are the stator and the rotor. The stator can be built by the permanent magnet or by energising the coil to behave as the magnet. This stator is connected directly to the output side of the generator that produced the electricity. The arrangement of the rotor is in a rotating mechanism where it is built from toothed segments that can be separated between two poles. The rotor is placed inside the stator between the air-gap and it rotates when the external force is applied to it. The synchronous generator can be represented in electrical equivalent circuit which is easier to understand and is shown in Figure 3.2. It shows that, the field winding $L_{f}$ is energised by the dc source current $i_{f}$. The armature windings $L_{a}, L_{b}$ and $L_{c}$ represent the stator elements with the current flowing out from the generator. When it rotates, the magnetic flux is induced and it will create the electromagnetic voltage $e$ that will appear in the output voltage of $v_{a}, v_{b}$ and $v_{c}$.

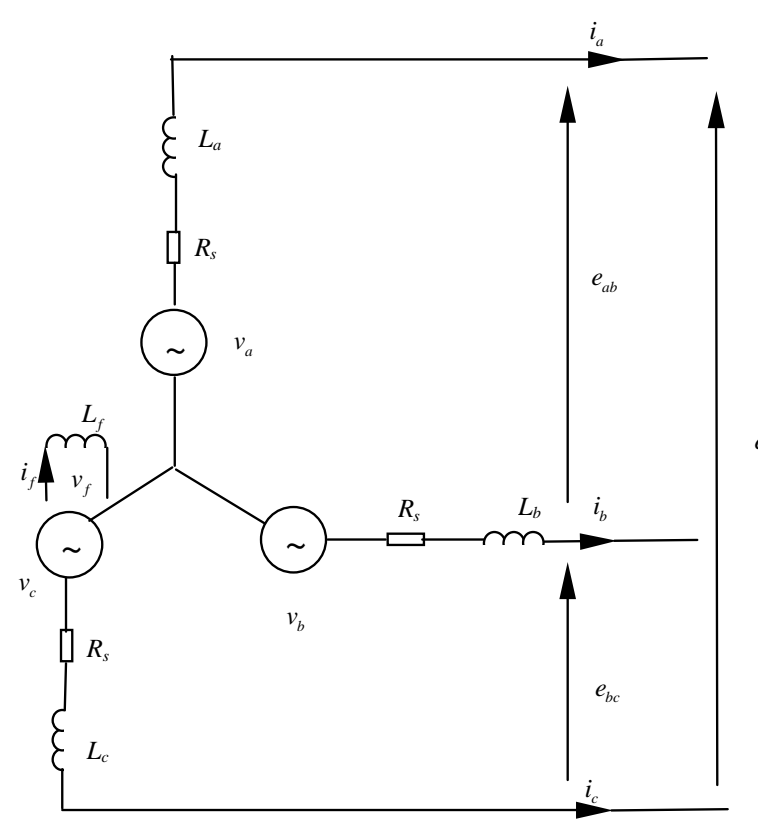

Figure 3.2: The circuit diagram of the 3-phase synchronous generator equivalent model

This general explanation of the synchronous generator is used, in order to understand the working principle of the linear generator. As is known, the linear generator has a same working principle as the synchronous generator but the different is in the structure of the stator and rotor. The main differences from the synchronous generator and the LG are the torque is changed by the force and the phase angle $(\theta)$ is changed to the $(z)$ direction in the LG. These differences are shown in Figure 3.3. Here, it shows that the direction of the magnetic field in the rotating machine is in circle where it represent by the $\theta$ while for the LG, the direction of the magnetic field is in the forward direction 
which is given by $z$. This explain that why the LG can be modelled from the rotating machine.

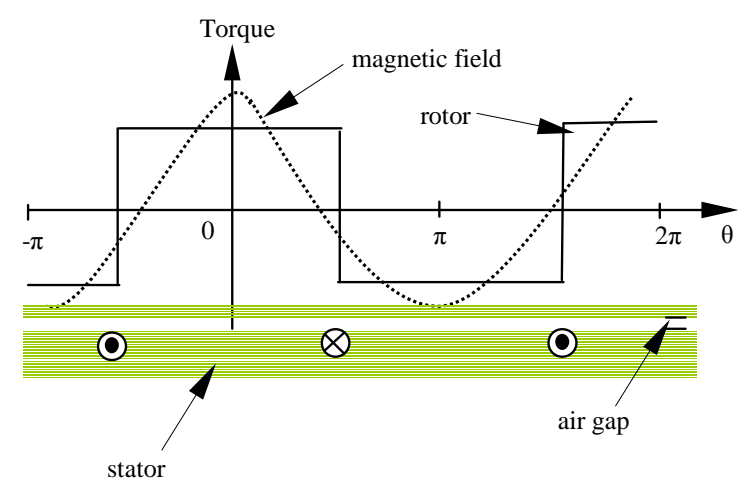

(a) direction of magnetic field in rotating machine $\theta$

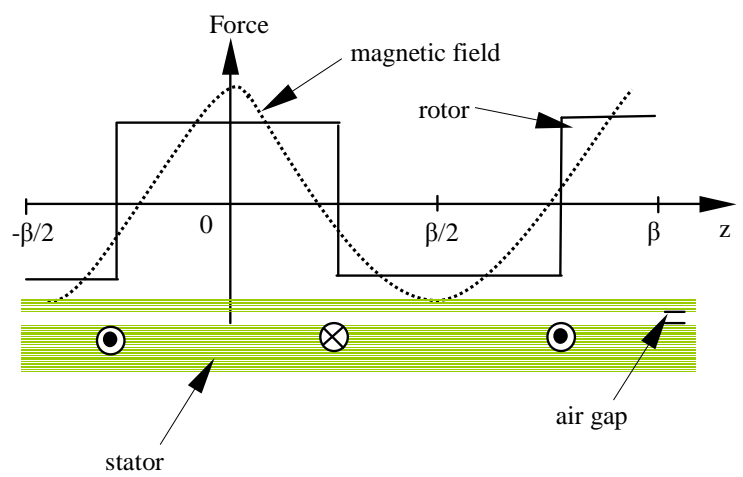

(b) direction of magnetic field in LG machine $z$

Figure 3.3: The direction of magnetic field between the rotating machine and LG in one full pitch

\subsection{Linear generator and diode rectifier for electrical power generation}

Figure 3.4 shows the arrangement of the LG, the diode and the aircraft itself in order to convert the kinetic energy to electrical energy. This arrangement is known as direct drive power take-off, and has been applied to convert the energy from the ocean wave into the electrical energy $[30,11,35,36]$. This is the simplest arrangement for the LG in the ERLA system before it can be connected to other parts of the power conditioning devices. This configuration will not use any control strategy in order to minimise the number of the controllers that will be implemented in the ERLA system. The control strategies that can be applied to the power conditioning devices will explain in the next 
chapter, where at the same time this controller can also control the outputs from the LG.

In Figure 3.4, the outputs of the LG are the excitation voltage given by $v_{a}, v_{b}$ and $v_{c}$ and the electromagnetic force $F_{e}$, known as the braking force. This $F_{e}$ has a relationship with the output current of LG given by $i_{a}, i_{b}$ and $i_{c}$ where it is proportional to the current. The relationship between the braking force and the force generated by the aircraft can be given as:

$$
F+F_{e}=F_{m}
$$

where $F$ is the force from the aircraft and $F_{m}$ is the losses force applied to the LG. For an ideal case, $F_{m}$ is assumed to be zero and it will make $F_{e}$ equivalent to $F$. As the result, the profile and amount of $F_{e}$ will determine the comfort of the passenger in the aircraft. For example, if this force has a linearly increasing profile, at some point the passenger will feel a high pressure impact to the body, that will make them feel uncomfortable, and when this force is maintained constant, the discomfort can be avoided. This condition will be explained more in the next chapter when this force can be controlled.

The advantages of using the LG compared to the synchronous generator are due to the structure of the LG itself which is in flat and also the wave of the magnetic flux is travelling in the same direction of the speed. The parameters of the speed $v$ and the displacement $z$ are needed in order to model the LG that is responded to the landing. It is when the phase angle in the rotating machine $\theta$ is changed to the $z$ in the LG. The relation between these two components are given by:

$$
\theta=\frac{2 \pi z}{\beta}
$$

where $\beta$ is the wavelength that relates to the total winding length in the LG stator.

Figure 3.4 also consists of external inputs to the LG which are the induced currents $i_{a}, i_{b}, i_{c}$ and the constant armature reaction $m_{f} i_{f}$. Due to the $v$ effect, the voltage and current outputs from the LG may have variable magnitude and frequency which are not suitable to be used if no power conditioning devices and control strategy are been applied. The diode rectifier is used to change the variable current, voltage and frequency to the dc voltage and current. Here, the generated dc outputs are easier to control and to maintain by using suitable power conditioning modules. No control strategy has been applied to the LG in order to obtain the full advantage of the LG itself. 


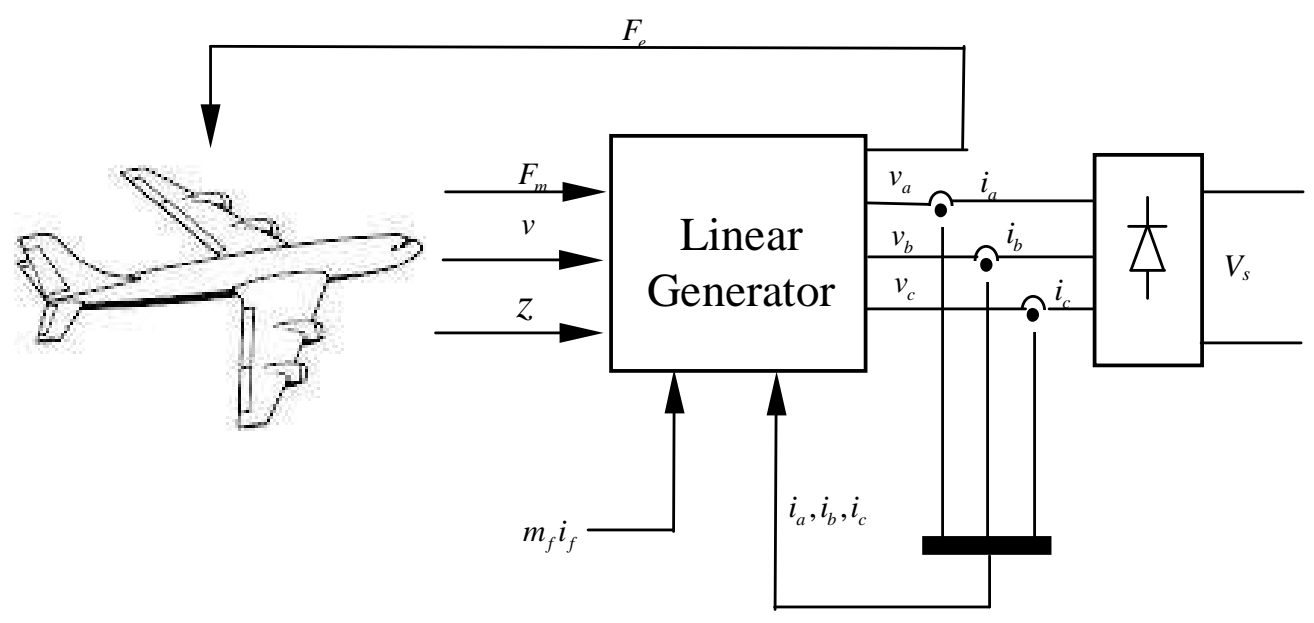

Figure 3.4: The block diagram of energy conversion without the dc-dc converter

\subsection{Modelling of the linear generator}

To analyse the LG operation, the equivalent circuit for the synchronous generator is taken into consideration $[125,126]$. As is known, the inputs of the LG are the $v$ and $z$, while the output is the electrical power. Due to these parameters, the LG can be modelled in two parts: the mechanical part and the electrical part. For the mechanical part, the model is based on equation 3.2 which reflects to the landing process, while for the electrical part, two models can be modelled, based on the equivalent model of the LG. The models are:

- the 3-phase electrical system model [127, 128, 129]

- the vector oriented method ( $d q$ transformation) model $[23,7,130,131,111]$

The 3-phase electric circuit diagram is used in order to model the LG shows in Figure 3.2. This diagram is based on the 3-balanced system that consists of phase $a, b$ and $c$. For the vector oriented method it can be modelled by changing the 3-phase diagram in Figure 3.2 to the $d q$ phase model using the $d q$ transformation. The $d q$ model of the machine has been introduced as the comparison for the 3-phase to deal with the mathematical model complexity and to increase the simulation time.

\subsubsection{Linear generator model in 3-phase system}

The details of the windings can be referred to $[125,126,132]$. In this model, the generator is assumed to be balanced and all the stator inductances are taken as constant 
due to the round rotor machine. In order to make an ideal synchronous generator behave like an ideal linear generator with no magnetic saturation effects and no eddy currents inside the generator, a pair of poles have been used at the phase and the rotor with a uniform air gap. The stator winding creates a self inductance and mutual inductance to other phases. Figure 3.5 shows the structure of an idealised three phase round machine that will be used to determine the LG equations.

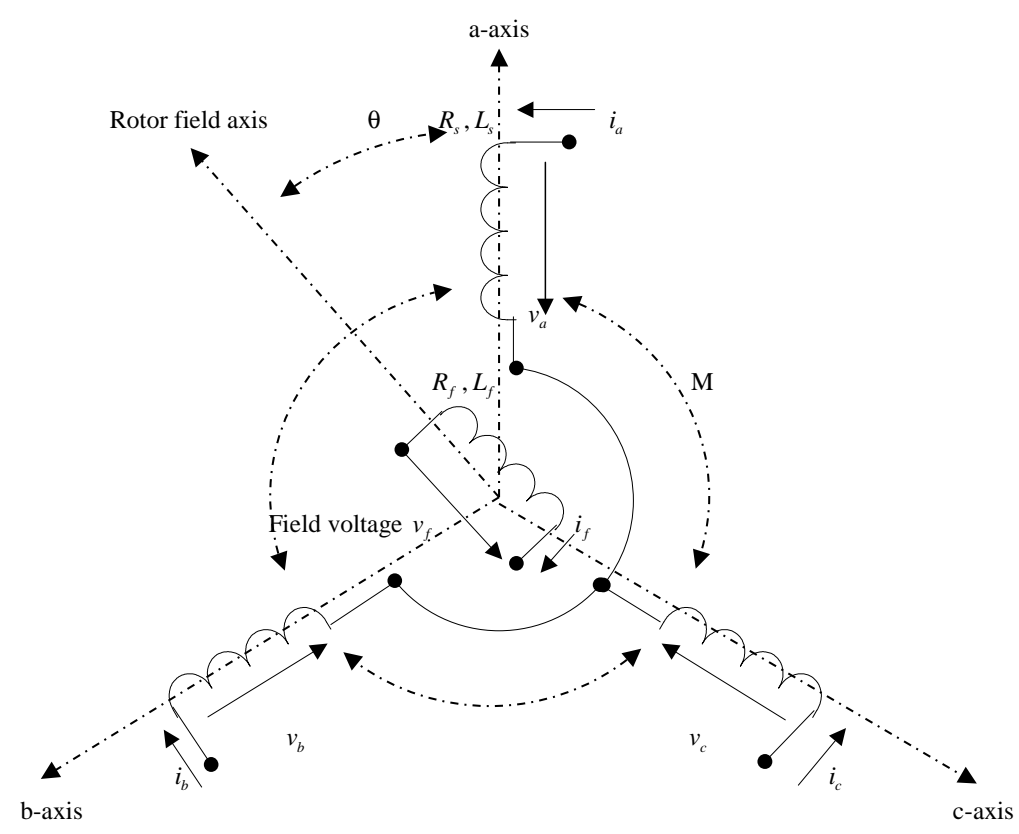

Figure 3.5: The ideal three-phase generator in rotating mode

There are $a b c$ axes that represent the phase position in the balanced system which is given in the anti-counter-clockwise system. The phase angles are given by:

$$
\begin{aligned}
& \theta_{a}=0^{0} \\
& \theta_{b}=-120^{0} \\
& \theta_{c}=-240^{\circ} .
\end{aligned}
$$

Each axis consists of the self inductance and the mutual inductance. The self inductances are given by:

$$
L=L_{a}=L_{b}=L_{c}
$$

while the mutual inductances are given by:

$$
-M_{s}=L_{a b}=L_{b c}=L_{c a}
$$

The rotor winding, known as the field winding, gives an inductance effect to the 
stator windings by the rotor position $\theta_{a}, \theta_{b}$ and $\theta_{c}$. The mutual inductance between the field winding and each of the three stator windings are given by:

$$
\begin{aligned}
L_{a f} & =m_{f} \cos \theta \\
L_{b f} & =m_{f} \cos \left(\theta_{d}-120^{0}\right) \\
L_{c f} & =m_{f} \cos \left(\theta_{d}-240^{0}\right) .
\end{aligned}
$$

The complete flux linkages with armature and field windings can be written as:

$$
\begin{aligned}
\lambda_{a} & =L_{a} i_{a}-M_{s}\left(i_{b}+i_{c}\right)+L_{a f} i_{f} \\
\lambda_{b} & =L_{b} i_{b}-M_{s}\left(i_{a}+i_{c}\right)+L_{b f} i_{f} \\
\lambda_{c} & =L_{c} i_{c}-M_{s}\left(i_{a}+i_{b}\right)+L_{c f} i_{f} \\
\lambda_{f} & =L_{a f} i_{f}+L_{b f} i_{f}+L_{c f} i_{f}+L_{f} i_{f} .
\end{aligned}
$$

If the phase current in $i_{a}, i_{b}$ and $i_{c}$ are balanced as given by:

$$
i_{s}+i_{b}+i_{c}=0 \text {. }
$$

By substituting equation 3.8 into equation 3.9, the simplified flux linkage equations can be written as:

$$
\begin{aligned}
\lambda_{a} & =\left(L_{a}+M_{s}\right) i_{a}+L_{a f} i_{f} \\
\lambda_{b} & =\left(L_{b}+M_{s}\right) i_{b}+L_{b f} i_{f} \\
\lambda_{c} & =\left(L_{c}+M_{s}\right) i_{c}+L_{c f} i_{f} .
\end{aligned}
$$

To give a relation between the flux linkage and the mutual inductance for the field winding, equation 3.7 can be substituted into equation 3.10 where the equation can be written as:

$$
\begin{aligned}
& \lambda_{a}=L i_{a}+m_{f} i_{f} \cos \left(\omega t+\theta_{a}\right) \\
& \lambda_{b}=L i_{b}+m_{f} i_{f} \cos \left(\omega t+\theta_{b}\right) \\
& \lambda_{c}=L i_{c}+m_{f} i_{f} \cos \left(\omega t+\theta_{c}\right),
\end{aligned}
$$

where $L=\left[L_{a}\right.$ or $L_{b}$ or $\left.L_{c}\right]+M_{s}$. From this equation, the flux linkage has two flux components which are the armature and the field winding. From Figure 3.2, each phase 
has a resistor $R$ and the voltage from across the terminal which the current flowing out is given by:

$$
v=-R i-\frac{\delta \lambda}{\delta t}
$$

By substituting equation 3.12 into equation 3.11 the voltage at the terminal can be given by:

$$
\begin{aligned}
v_{a} & =-R i_{a}-L \frac{\delta}{\delta t} i_{a}+\omega m_{f} i_{f} \sin (\omega t) \\
v_{b} & =-R i_{b}-L \frac{\delta}{\delta t} i_{b}+\omega m_{f} i_{f} \sin \left(\omega t-120^{0}\right) \\
v_{c} & =-R i_{c}-L \frac{\delta}{\delta t} i_{c}+\omega m_{f} i_{f} \sin \left(\omega t-240^{0}\right) .
\end{aligned}
$$

From these equations, the voltage terminals consist of the voltage at $R$ and $L$ but also includes the generated voltage. By selecting $R=L=0$, in order to transmit all the power to the load, the generated voltage can be given by:

$$
\begin{aligned}
& v_{a}=e_{a}=\omega m_{f} i_{f} \sin (\omega t) \\
& v_{b}=e_{b}=\omega m_{f} i_{f} \sin \left(\omega t-120^{0}\right) \\
& v_{c}=e_{c}=\omega m_{f} i_{f} \sin \left(\omega t-240^{0}\right) .
\end{aligned}
$$

From these equations, the terminal voltages are dominated by the generated voltage that is proportional to the rotor speed $\omega$ in the LG structure. From the LG concepts the $\theta$ is proportional to the $z$ which gives the $\theta$ as:

$$
\theta=\frac{2 \pi z}{\beta}=\omega t
$$

With some simplification of equation 3.15 , the $\omega$ can be written as:

$$
\begin{aligned}
\theta & =\omega t \\
\frac{2 \pi z}{\beta} & =\omega t \\
\frac{2 \pi z}{\beta t} & =\omega \\
\frac{2 \pi v}{\beta} & =\omega .
\end{aligned}
$$

By taking equations 3.14 and 3.16, the mathematical model for the first model can be designed in the simulink environment where shows in Figure 3.6.

From Figure 3.6, the outputs of the linear generator are voltage $e_{a}, e_{b}, e_{c}$, braking force $F_{e}$ and the power $p$. For the LG machine, the output power is determined by the 
$F_{e}$ generated inside the LG. The power can be calculated by using the basic equation of the power as $p=v_{a b c} \times i_{a b c}$ and it is given by:

$$
p=\left[-R i_{a b c}-L \frac{\delta}{\delta t} i_{a b c}+\omega M_{f} i_{f} \sin \left(\omega t+\theta_{a, b, c}\right)\right] i_{a b c} .
$$

As noted, the value for $R$ and $L$ have been ignored in order to transfer all the energy from the generator to the system which makes the final equation can be written as:

$$
p=\left[\omega M_{f} i_{f} \sin \left(\omega t+\theta_{a, b, c}\right)\right] i_{a b c} .
$$

From equation 3.2, the $F_{e}$ has the same magnitude as the $F$, but in opposite sign, which indicates that the $F_{e}$ is applied in the opposite direction of the landing. The force during landing can be given as:

$$
F=m a=m \frac{d v}{d t}
$$

where $m$ is the mass of the aircraft and $a$ is the deceleration given in $\frac{m}{s^{2}}$.

To obtain the relationship between the force and the power generated by the LG, the work equation which relates to the power is used:

$$
p=\frac{\delta W}{\delta t} .
$$

where $W$ is the work done and by expanding this equation, the relationship between the force and power can be introduced as:

$$
\begin{aligned}
& p=\frac{\delta W}{\delta x} \times \frac{\delta x}{\delta t} \\
& p=F v .
\end{aligned}
$$

By substituting equation 3.18 into equation 3.21, the force can be calculated as:

$$
\begin{aligned}
& {\left[\omega m_{f} i_{f} \sin \left(\theta_{w}\right)_{a b c}\right] I_{a b c}=F v} \\
& \frac{\left[\omega m_{f} i_{f} \sin \left(\theta_{w}\right)_{a b c}\right] I_{a b c}}{v}=F .
\end{aligned}
$$

The three phase force generated from the LG can be derived as:

$$
F_{e}=\frac{\omega}{v} m_{f} i_{f}\left[\begin{array}{c}
\sin \left(\omega t+0^{0}\right) \\
\sin \left(\omega t-120^{0}\right) \\
\sin \left(\omega t-240^{0}\right)
\end{array}\right]\left[\begin{array}{c}
i_{a} \\
i_{b} \\
i_{c}
\end{array}\right] .
$$

Equation 3.22 is used to model the block diagram of the force in the 3-phase model. It shows that the $F_{e}$ is proportional to the current flowing out from the generator terminal and inverse to the speed. In this case, the speed cannot be controlled but the control parameter is the current flowing out that will change the force profile at the 
same time. This relationship gives an idea on how to control the LG by controlling the current; this will be explained and shown in the next chapter of the energy conversion.

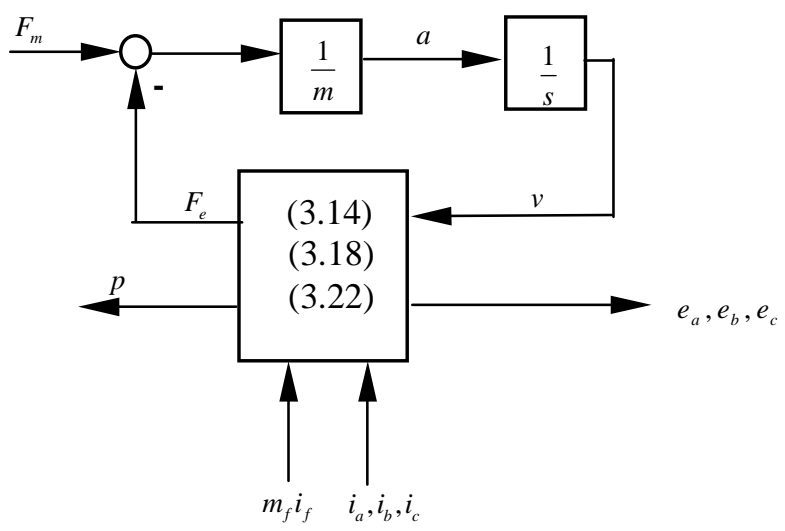

Figure 3.6: The model of linear machine in simulink block diagram for 3 phase analysis

\subsubsection{Linear generator model in $d q$ transformation model}

The model of the LG can also be designed using the $d q$ transformation [126] for easier mathematical computation and analysis [23] and also to respond to the transient effect [125] if the unbalanced system happens to the LG. The $d q$ model means that the axes in the synchronous generator are transferred to two axis components which are known as the direct axis $d$ and the quadrature axis $q$. Here, the zero sequence is not taken into consideration. This concept is much easier to analyse and develop because all the time-varying state variables become dc time invariant where only one operating point needs to be defined and considered [126] compared to the 3-phase model where it requires a very small integration step even in the steady state operation which could create computational problems (long simulation time and insufficient memory for storing simulation history in the workspace). Figure 3.7 shows the $d q$ format for the synchronous generator with the field winding of the rotor.

The linear generator is modelled in a $d q$ reference frame where the relationship between a set of $d q$ and the corresponding set of 3-phase $a b c$ variables are provided by the equation below and by using the Park's transformation equation given by:

$$
\begin{gathered}
\operatorname{Park}^{\prime} s=\frac{\sqrt{2}}{3}\left[\begin{array}{cc}
\cos (\omega t) & -\sin (\omega t) \\
\cos \left(\omega t-120^{0}\right) & -\sin \left(\omega t-120^{0}\right) \\
\cos (\omega t-240) & -\sin (\omega t-240)
\end{array}\right]^{T} \\
{\left[X_{d q}\right]=\operatorname{Park}^{\prime} s\left[X_{a b c}\right],}
\end{gathered}
$$

and the inverse transform of equation 3.23 is given by: 


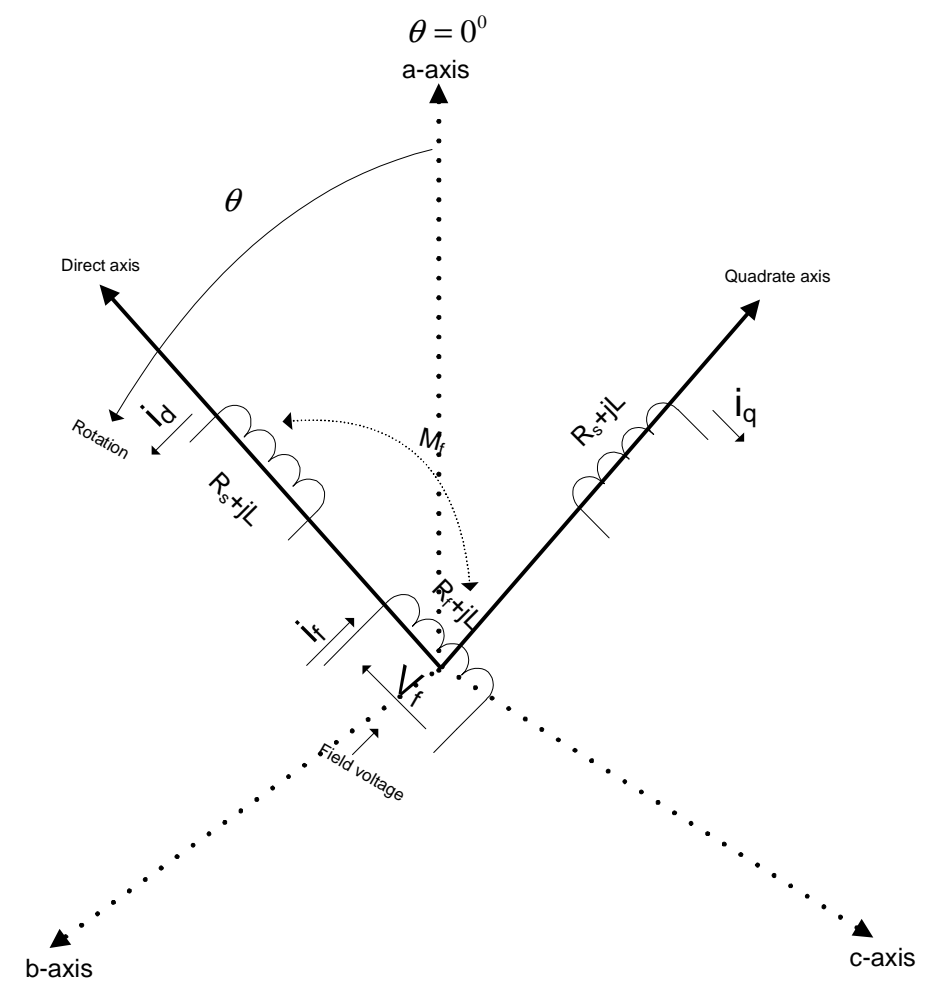

Figure 3.7: Representing the direct-axis winding of synchronous generator after the dq transformation

$$
\begin{gathered}
{\left[\begin{array}{l}
a \\
b \\
c
\end{array}\right]=\left[\begin{array}{cc}
\cos (\omega t) & -\sin (\omega t) \\
\cos \left(\omega t-120^{0}\right) & -\sin \left(\omega t-120^{0}\right) \\
\cos \left(\omega t-240^{0}\right) & -\sin \left(\omega t-240^{0}\right)
\end{array}\right]\left[\begin{array}{l}
d \\
q
\end{array}\right]} \\
{\left[X_{a b c}\right]=\operatorname{Park}^{\prime} s^{-1}\left[X_{d q}\right] .}
\end{gathered}
$$

By using equation 3.14 from the three phase analysis, this 3-phase format can be changed to the $d q$ format by using equation 3.24. The following equation describes the transformation made to generate the $d q$ voltage:

$$
\begin{gathered}
{\left[\operatorname{Park}^{\prime} s^{-1}\left[\begin{array}{l}
e_{d} \\
e_{q}
\end{array}\right]=m_{f} i_{f}\left[\begin{array}{c}
\cos (\theta) \\
\cos \left(\theta-120^{0}\right) \\
\cos \left(\theta-240^{0}\right)
\end{array}\right]\right]^{T}} \\
{\left[\begin{array}{c}
e_{d} \\
e_{q}
\end{array}\right]=\omega m_{f} i_{f}\left[\begin{array}{c}
\cos (\theta) \\
\cos \left(\theta-120^{0}\right) \\
\cos \left(\theta+240^{0}\right)
\end{array}\right] \times \text { Park }^{\prime} s,}
\end{gathered}
$$

where, 


$$
\begin{aligned}
{\left[\begin{array}{l}
e_{d} \\
e_{q}
\end{array}\right] } & =\omega m_{f} i_{f}\left[\begin{array}{c}
\sin (\omega t) \\
\sin \left(\omega t-120^{0}\right) \\
\sin \left(\omega t-240^{0}\right)
\end{array}\right]\left[\begin{array}{lll}
\cos (\omega t) & \cos \left(\omega t-120^{0}\right) & \cos \left(\omega t+120^{0}\right) \\
\sin (\omega t) & \sin \left(\omega t-120^{0}\right) & \sin \left(\omega t+120^{0}\right)
\end{array}\right] \\
& =\omega m_{f} i_{f}\left[\begin{array}{c}
0 \\
1.5
\end{array}\right] .
\end{aligned}
$$

where $\omega$ is the same as in equation 3.16, and the voltages $e_{d q}$ are the armature $d$ and $q$ axis of the generated voltage. The final equation can be written as:

$$
e_{d}=0,
$$

and

$$
e_{q}=1.5 \omega m_{f} i_{f} .
$$

The power for the $d q$ components can be calculated by referring to equation 3.28 which it consists of speed and current information. The $d q$ power equation can be written as:

$$
\begin{aligned}
p & =v_{a b c} \times i_{a b c} \\
p & =V_{d} i_{d}+V_{q} i_{q} \\
p & =\frac{3}{2}\left[1.5 \omega m_{f} i_{f}\right] i_{q} .
\end{aligned}
$$

By substituting equation 3.28 into equation 3.21 , the force generated by the machine in the $d q$ model can be determined as,

$$
F_{e}=\frac{9}{4} \frac{\omega m_{f} i_{f}}{V} i_{q} .
$$

It shows that the force is related to the $i_{q}$ components which is the output current from LG and it also explained by controlling this current the force will be controlled. The model block diagram for the $d q$ model can be shown in Figure 3.8.

\subsection{Simulation results}

In this section, the simulation results for both models are shown and discussed. The idea is to understand the behaviour of the LG outputs in order to change the kinetic energy from the landing aircraft into electrical energy, and at the same time to analyse the pattern of power output and force generated from the LG machine before any controller can be applied to it. For the simulation time, it is started at $t=0 \mathrm{~s}$ and ends at $t=5 \mathrm{~s}$. This simulation time can be changed if the actual value of the aircraft and the speed have been used. The simulations were carried out in MATLAB 8.0 with Simulink. 


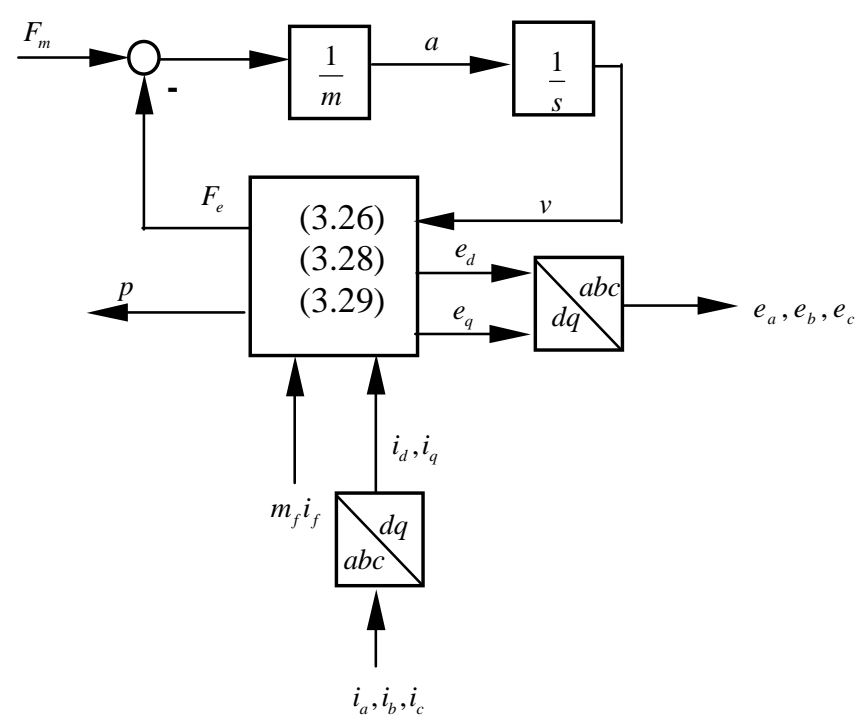

Figure 3.8: The model of linear machine in simulink block diagram for $d q$ analysis

The solver used in the simulation was the ode23tb with the maximum step size of 0.01 milliseconds.

Table 3.1 shows the parameters value that has been used to simulate the LG in order to understand the behaviour of the energy conversion from kinetic energy to electrical energy which happens in the LG.

Table 3.1: Parameters for the LG model

\begin{tabular}{|c|c|c|c|}
\hline Parameters & value & Parameters & value \\
\hline \hline speed & $10 \mathrm{~m} / \mathrm{s}$ & mass & $100 \mathrm{~kg}$ \\
\hline$E$ & $100 \mathrm{~V}$ & $R_{L G}$ & $0.01 \Omega$ \\
\hline
\end{tabular}

\subsubsection{Linear generator with 3 -phase model}

Figures 3.9 and 3.10 show the outputs from the LG that was designed using the 3 -phase model. Here, the figures can be separated into two categories: the electrical outputs and the mechanical outputs. The electrical parts are shown in Figures 3.9a to 3.9c. The first two figures show the current and voltage outputs that have variable magnitude and frequency changed along the simulation time, which is caused by the decreasing speed of the aircraft. For the power output profile it is shown in Figure 3.9c, where this power was collected at the rectifier output. It shows that the power is in exponential decay that only can provide the power in very short period of time.

Figures $3.10 \mathrm{a}$ and $3.10 \mathrm{c}$ are about the deceleration and distance of the LG. Figure $3.10 \mathrm{~b}$ shows the force response at the LG. It shows that the maximum force is induced 
at very short of time in the beginning and then it decrease to zero. This is uncontrolled force and make the system unstable. Regarding to equation 3.19, the control variable is the deceleration. By controlling this deceleration, the force profile will change and also it will change the profile of the power generated by the LG. From equations 3.22 and 3.29, the force is also related to the current at the terminal voltage of the LG. This shows that these three parameters, which are the power, force and deceleration, are related to each others. The distance graph shows how long the LG should be used if the system needs to be built.

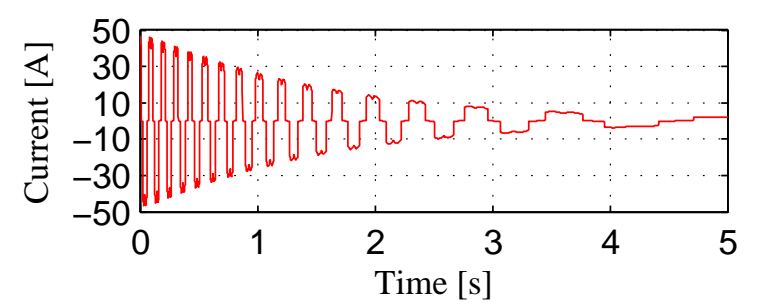

(a) current at rectifier input

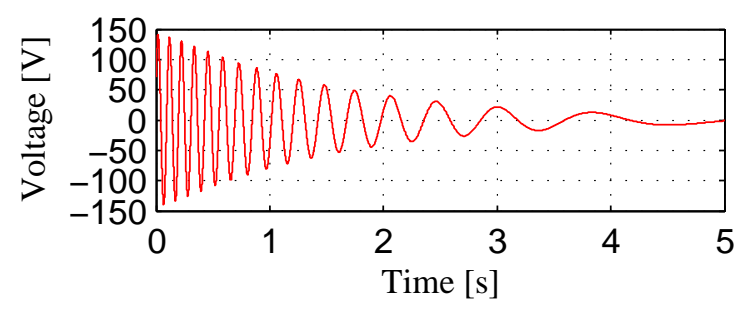

(b) voltage at rectifier input

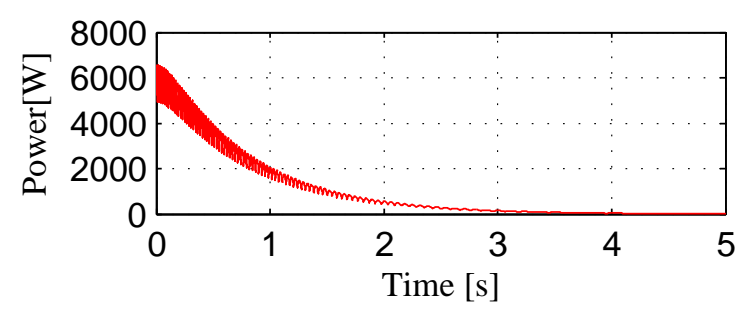

(c) power generated at the rectifier output

Figure 3.9: Simulation results for 3-phase linear generator model at the diode rectifier 


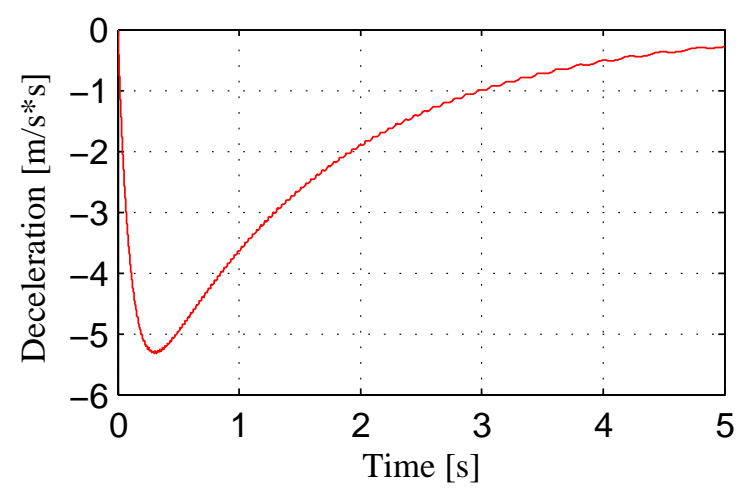

(a) acceleration speed profile during landing

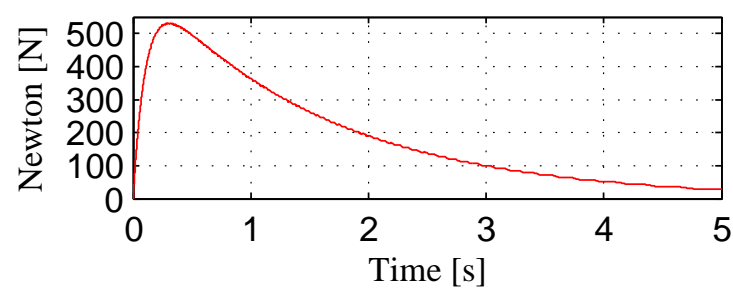

(b) $F_{e}$ (braking force) from LG during landing

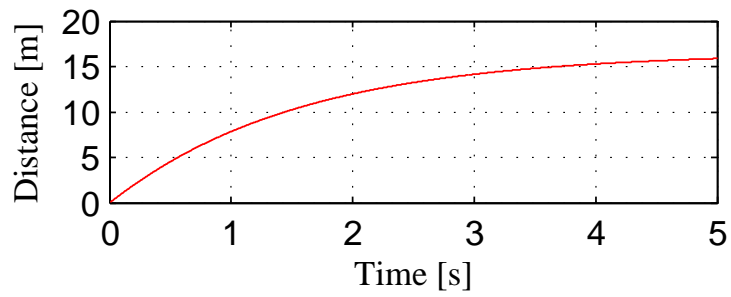

(c) distance covered by the LG during landing

Figure 3.10: Simulation results for 3-phase linear generator model at LG outputs

For the generated output power during landing, it is shown in Figure 3.11. It shows that, the power is in transient mode where it has high power at the beginning and continue to have exponential decay along this simulation. This power profile is related to the force output from the LG that is given in Figure 3.10b. This is the uncontrolled power, and it is not suitable to be applied to the electrical network or it will cause damage to the electrical system.

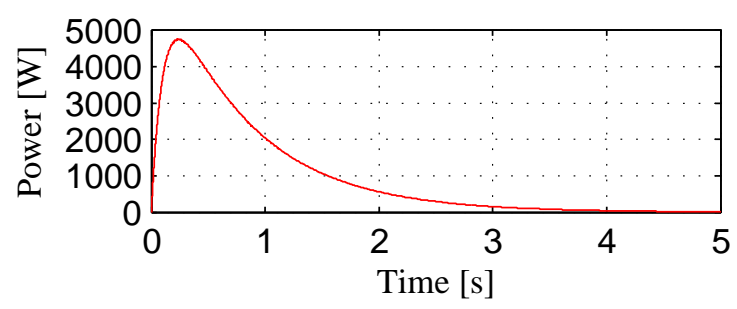

Figure 3.11: Generated power during landing for 3-phase model 


\subsubsection{Linear generator with $d q$ transformation model}

The second analysis of the LG model was conducted using the $d q$ transformation model that is shown in equation 3.26 to equation 3.29. The same simulation time was used in order to compare the results with the 3-phase model. Figures 3.12 and 3.13 show the outputs for this $d q$ model. From this model, again, all the results are the same with the results form the 3-phase model but there is a small increase in the magnitude of power and force. These are caused by the zero sequence, which was not included in the model design. Basically, this zero sequence model will help the $d q$ model to behave like the 3-phase model. As a result this $d q$ model can also be used in modelling the LG generator structure.

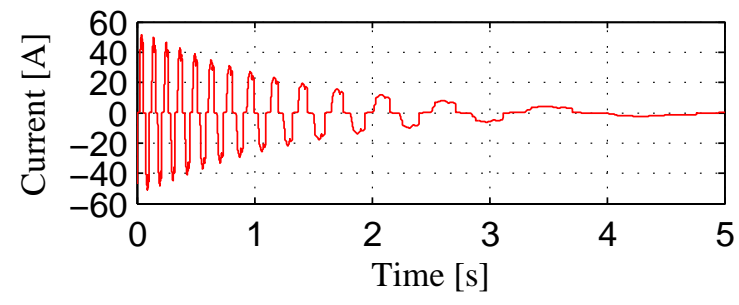

(a) current at rectifier input

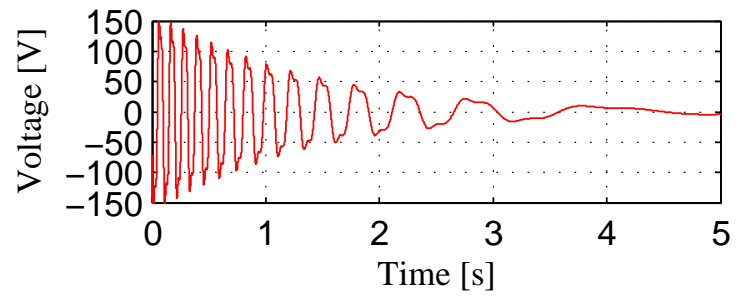

(b) voltage at rectifier input

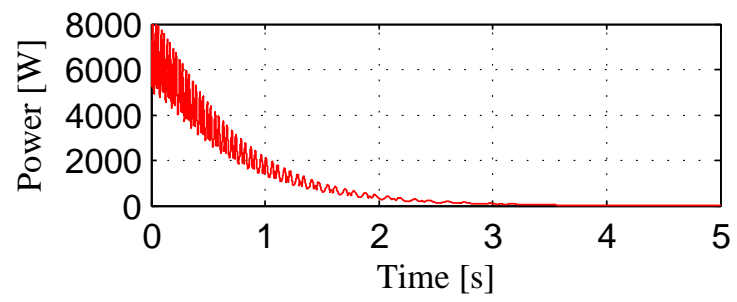

(c) power generated at rectifier output

Figure 3.12: Simulation results for dq linear generator model at the diode rectifier 


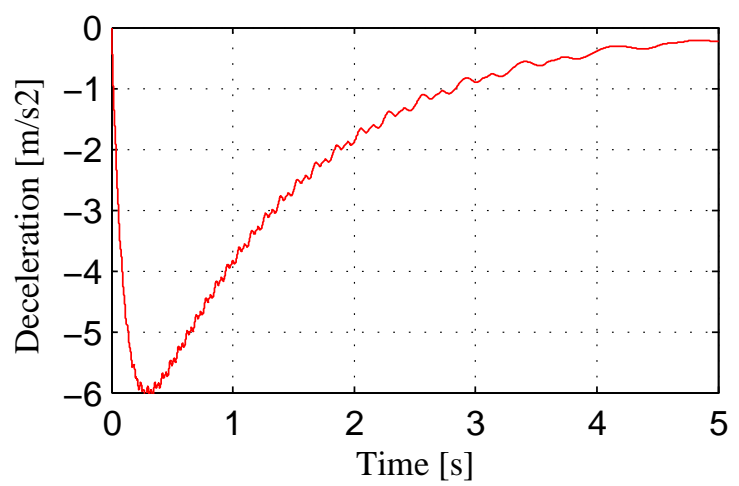

(a) acceleration speed profile during landing

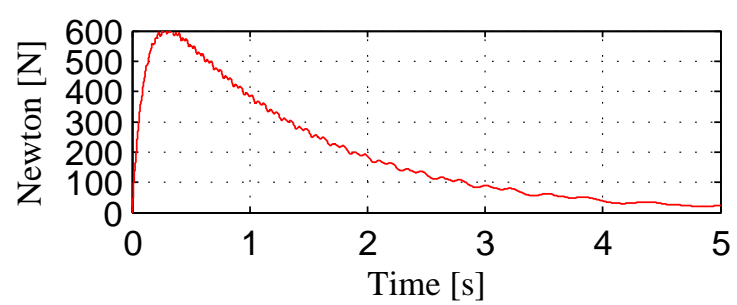

(b) $F_{e}$ (braking force) from LG during landing

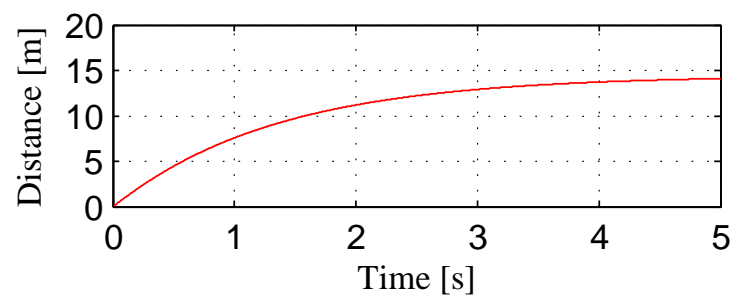

(c) distance covered by the LG during landing

Figure 3.13: Simulation results for dq linear generator model at LG outputs

Figure 3.14 shows the output of the generated power at the LG during landing for the $d q$ model. It also gives fast transient response due to the force output generation. In this case, it indicates that the power profile can be changed if the force profile can be changed by controlling the input current.

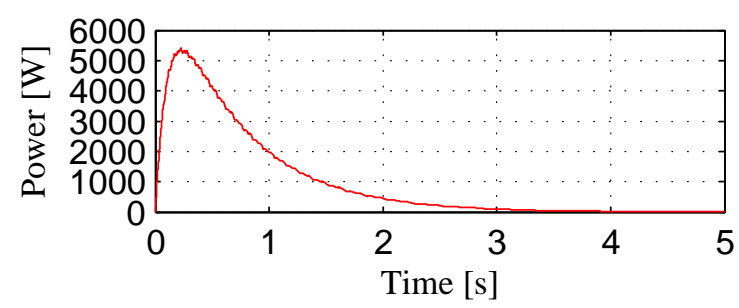

Figure 3.14: Generated power during landing for $d q$ model 


\subsection{Concluding remarks}

As a conclusion, the LG generator and diode rectifier are used to convert the kinetic energy from landing aircraft into electrical energy. This is because, the LG can be placed on the runway due to the structure which is a flat. The stator of the LG can be put underneath the runway, while the rotor can be a platform in order to park the aircraft during landing or as a pushing device which are shown in Figures 2.5 and 2.6. For the LG operation, it uses the speed and creates the force and this is the reason why the LG is suitable for the ERLA application. The LG output generates voltage, current and power output. The output force is the main component in the LG system which it will determine the generated power during landing. In order to see all these effects, the LG can be modelled using the 3-phase model or the $d q$ transformation model. Both of the models use the speed as the input and create a force as one of the outputs. This force is really important in the ERLA application where it contributes to the passengers comfort. By controlling this force, the passenger discomfort can be minimised, but to control this force a control strategy needs to be modelled for the LG. By finding the relationship between the force and the generated current from the LG, the control strategy can be applied at the power conditioning device. This means that by controlling the current at the power conditioning devices it will control the force, power and deceleration at the LG outputs. With no control strategy applied to the LG, the build up cost can be reduced. Finally, both models show the same behaviour and it can be used to understand the effect of the outputs during landing. From the results it also show that all the outputs have an exponential decay, which will make the power generated behave like an impulse power in a short period of time; this is not good for power distribution applications when it been connected to the electrical network. To regulate these outputs, power conditioning device is necessary and the next chapter will explain the control application that have been used in order to change this exponential decaying power profile to a more useful power profile by controlling the output current of the diode rectifier. 


\section{Chapter 4}

\section{Energy conversion: The $H^{\infty}$ current control design for generated outputs}

This chapter explains the controller design that has been applied in the energy conversion. In order to control the deceleration, force and power at the LG side, a cascaded buck-boost (CBB) converter is used in order to control the input current. The current is proportional to the deceleration and it will offer a constant force at the LG outputs. This constant force will ensure the passengers in the aircraft will not experience any uncomfortable conditions compared to normal landing. Another reason is that, this current is also proportional to the deceleration of the LG, where it change the profile of the generated power generated from exponential decay to linearly decreasing power. This linearly decreasing profile is the suggested condition proposed in [9] for harvesting the energy from landing aircraft. The $H^{\infty}$ control theory has been used in order to determine the compensator controller value for the current control that is suitable with variable input voltage which is appeared at the input at the CBB converter. After the value of the compensator has been found, the stability analysis needs to be conducted in order to ensure the $H^{\infty}$ performance is in a stable condition that will give of better dynamic response. At the end of this chapter, the simulation results regarding the CBB converter combined with the LG part have been used to control the input current at the CBB converter. In the mean time, it is also able to control the outputs of the LG for the complete energy conversion process.

\subsection{The model and parameters calculation of the CBB con- verter}

Figure 4.1 shows the arrangement of the CBB converter with in the energy conversion. The CBB converter has been used because it gives low stress components and provides 
the energy path [61] from the input to the output. The CBB converter is the combination of the buck and boost converter. The operation of the CBB converter can be summarised: when the input voltage at the CBB converter $\left(V_{s}\right)$ is higher than the CBB converter output voltage $\left(V_{o}\right)$, the $\mathrm{CBB}$ converter will operate in the buck operation mode, and when the $V_{s}$ is lower than $V_{o}$, the boost operation mode takes place. These two modes are controlled by the two switches which are $S_{1}$ and $S_{2}$. As known, the output from the diode rectifier contains high ripple current and voltage due to non control element. Due to this a high ripple current will flow to the inductor $\left(L_{c b b}\right)$ and the value for the inductor must be chosen wisely. In order to regulate the power, force, deceleration and the input current, the $\mathrm{CBB}$ converter needs to be controlled using current control. Before the controller can be applied to the CBB converter, the value for the inductance and capacitance need to be determined in order to hold the energy generated during the landing.

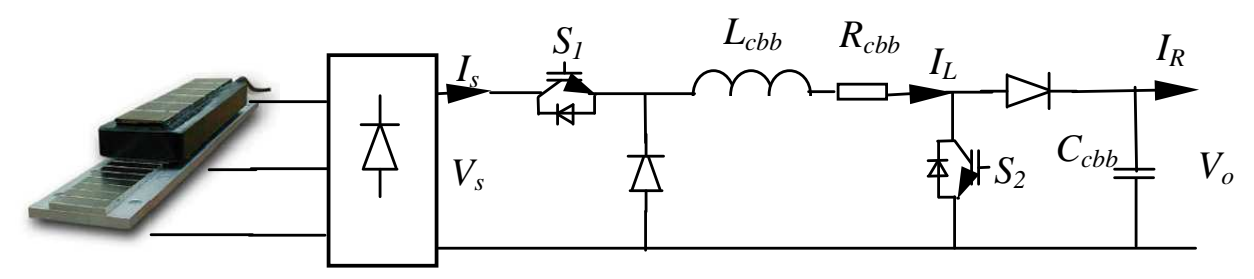

Figure 4.1: The circuit diagram of CBB converter in ERLA system

Several control strategies can be used in the CBB converter, such as the voltage control and the current control. For the case of the ERLA configuration, the current control which is used the input current $\left(I_{s}\right)$ has been selected. Regarding to [16], $I_{s} \approx I_{L}$ at the diode output current and it relates to the generated force $\left(F_{e}\right)$ for the LG. It ensures that the force, the deceleration and the power in the previous part of energy conversion can be controlled using this current controller. The current controller arrangement is shown in Figure 4.2. As mentioned, the CBB converter is operated in two modes and these modes depend on the operations of the logic sequences which will be discussed later.

To calculate the $L_{c b b}$ and $C_{c b b}$ values for the CBB converter, the kinetic energy from the aircraft need to be determined. In [9], the actual value of the aircraft and the speed during landing have been chosen, but if these values are used in this work the simulation time to complete the whole process of the ERLA network is taken longer time and required greater memory allocation to store the data. Due to this restriction, the small ratio of the actual values is used. This ratio is good for this feasibility study in 


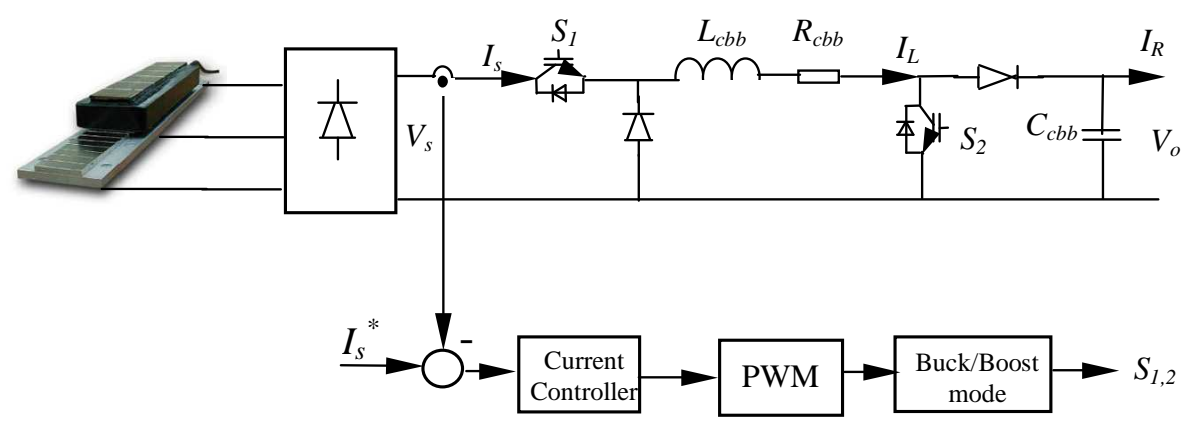

Figure 4.2: The current control strategy for CBB converter

order to understand the process of how the kinetic energy is produced until how much of the energy can can been transferred. In this work, the aircraft is represented by the flywheel that has been build in the lab where it has a weight of $m=100 \mathrm{~kg}$ and a speed of $v_{o}=10 \mathrm{~m} / \mathrm{s}$ in order to respond like the landing aircraft. The kinetic energy can be calculated as:

$$
E=\frac{1}{2} m v_{o}^{2}=5000 \mathrm{~J} .
$$

Equation 4.1 shows the generated kinetic energy during landing. If this energy needs to be transferred to the load within less than $4 \mathrm{~s}$, the power for a certain period of time can be given as:

$$
P=\frac{E}{t} .
$$

This power is known as the average power. By divided $E$ by $4 \mathrm{~s}$, the average power is calculated to be about $1250 \mathrm{~W}$. This average power needs to be transferred to the load in the same period as the landing time, or stored at the energy storage elements, or transferred to the grid for distribution. This power can be different depends to the size of the aircraft, where bigger aircraft will generate more average power in the same period of time. As mentioned before, the shape of the electrical power graph is determined by the generated force of the LG. In [9] it proposed two conditions which are: the constant deceleration and linearly increasing deceleration. These two decelerations give a different force output and power profile at the LG. For this research, the constant deceleration is gaining more interest due to the constant force that will be generated. This force is known as the braking force that is applied at the opposite direction of the moving force of the aircraft where it has direct impact to the passenger comfort. The 
force equation which is related to the deceleration is given by:

$$
F=m a
$$

where $m$ is the mass of the aircraft and $a$ is the deceleration speed in $\mathrm{m} / \mathrm{s}^{2}$. In order to obtain constant force, the deceleration must also be constant. Due to this condition, the electrical power equation can be arranged to:

$$
p=f \times v=f \times\left(v_{o}+a t\right)
$$

where $v=v_{o}+a t$ is known as the final speed and $p$ is the instantaneous power. Equation 4.3 is the equation to calculate the instantaneous power that has been explained in [9]. Assume, the deceleration is constant and is given by:

$$
a=\frac{10}{4}=-2.5 \mathrm{~m} / \mathrm{s}^{2} \text { (deceleration) }
$$

and the braking force for constant deceleration is given by:

$$
f=m a=100 \times 2.5=250 \mathrm{~N} .
$$

By substituting the constant deceleration into the final speed equation, the equation can be written as

$$
v=v_{o}+a_{o} t=10-2.5 t
$$

As a result, the power equation which responds to the time can be given by:

$$
p=f v=(m a)=250(10-2.5 t) .
$$

This power equation shows that the power from the landing is linearly decreasing with time, such that when $t=0 \mathrm{~s}$ the power is $2500 \mathrm{~W}$ and when $t=4 s$ the power is $0 \mathrm{~W}$. As calculated, the average power is about $1250 \mathrm{~W}$, the input current flows $\left(I_{s}\right)$ to the CBB converter can be calculated as given in the equation below is when the output at the dc-link voltage $\left(V_{o}\right)$ is maintained at $60 \mathrm{~V}$. This value is half of the generated voltage at the LG section:

$$
\begin{aligned}
P_{a v g} & =V_{d c} I_{L_{b b}} \\
1250 & =60 I_{L_{b b}} \\
20.8 & =I_{L_{b b}} .
\end{aligned}
$$

This value indicates that the amount of current needs to be controlled for the current controller in the CBB converter. The $L_{c b b}$ and $C_{c b b}$ value for the CBB converter can be 
calculated when the converter is in the buck mode. The reason why the buck mode has been chosen is because, when time $t=0 \mathrm{~s}$, the voltage and power generated by the LG are at the maximum value and it linearly reducing to the target output voltage of the $\mathrm{CBB}$ converter where it has been maintained at $60 \mathrm{~V}$. Equation 4.9 shows the equation to calculate the $L_{c b b}$ in the CBB converter when it is in the buck operation:

$$
L_{c b b} \ggg L_{\min }=\frac{(1-D)}{2 \times f} \times \frac{V_{d c}^{2}}{P_{a v g}},
$$

where $D$ is the maximum duty cycle due to the maximum generated voltage at the LG with the dc-link voltage which is given as $0.5, f$ is the switching frequency $(10 \mathrm{kHz})$ for the MOSFET devices and $V_{d c}$ is the voltage at the dc-link which set at $60 \mathrm{~V}$. By substituting these values into equation 4.9 the $L_{\min }=72 u H$ is found. The inductor value of $L_{c b b}=300 \mathrm{uH}$ has been selected for the ERLA simulation application. After the $L_{c b b}$ has been selected, the value for the $C_{c b b}$ can be calculated by referring to equation:

$$
r=\frac{1-D}{8 \times L_{c b b} \times C_{c b b} \times f^{2}},
$$

where $r$ is the ripple factor. To obtain a small ripple on the capacitor, the ripple must be chosen to be at $0.5 \%[133]$. The value for the capacitance can be calculated as:

$$
\begin{aligned}
0.005 & =\frac{1-D}{8 \times L_{c b b} \times C_{c b b} \times f^{2}} \\
C_{c b b} & =416.67 \mathrm{uF} .
\end{aligned}
$$

The value of $500 \mathrm{uF}$ has been chosen for the capacitor value in order to be used at the CBB converter.

\subsection{Design of $H^{\infty}$ current control for the CBB converter}

In this section, a current controller based on the $H^{\infty}$ control theory has been proposed for the CBB converter. As has explained, the objective is to control the deceleration speed in order to maintain the output force from the LG. In doing so, the input current to the $\mathrm{CBB}$ converter needs to be controlled. It is when the CBB converter is in the boost operation where the input voltage is lower than the output voltage. This will cause the inductor current to follow at the rated current value given in equation 4.8. It shows that by controlling the input current, it also can control the force. In this work, the $H^{\infty}$ control theory has been applied for the current controller, where it includes the disturbances and perturbation effects that give high robustness outputs, in order to obtain the minimum tracking error value. It also indicates that, it can respond to the changes in the inputs and outputs for the $\mathrm{CBB}$ converter. This is due to the 
arrangement of the CBB converter where it has the load current which contributes to the ineffectiveness design of the controller.

\subsubsection{Description of the control scheme}

For most MPPT concepts, the boost converter is used to control the maximum power and also the current flowing out from the generator. Due to this, the boost topology has been adopted in the ERLA application in order to achieve the target, and the proposed control scheme is shown in Figure 4.3. It adopts the $H^{\infty}$ current controller technique. In this figure, the feedback is the $I_{s}$, which is the output current from the diode rectifier. The error from the $I_{s}$ and the $I_{s}^{*}$ will form the input error to the $H^{\infty}$ controller block. The function of the $H^{\infty}$ block is to generate the desired signal for the pulse width modulation (PWM) block. These PWM signals are complementary to each switches that are determined by the logic sequence for buck or boost operation. The logic sequence is explained in the next subsection. Figure 4.3 shows the current control structure with the input current feedback loop at the CBB converter.

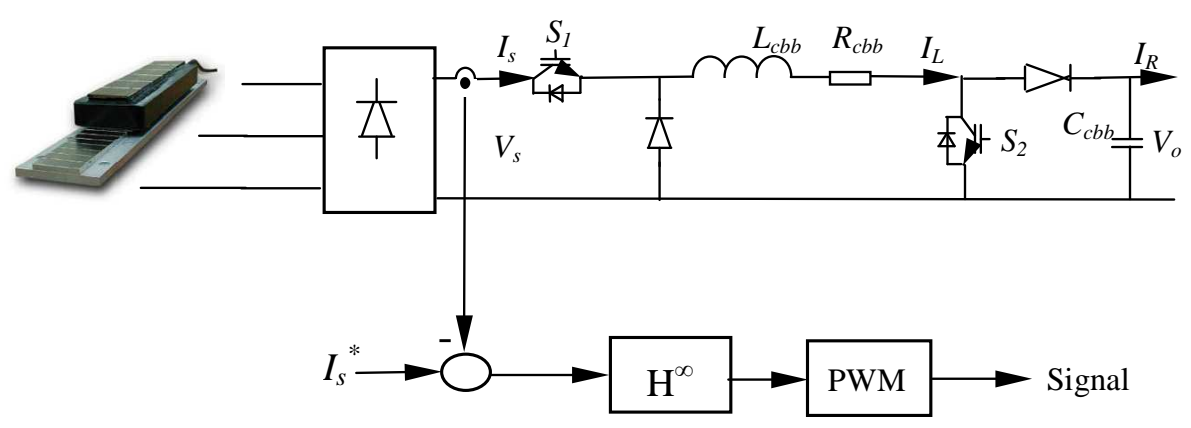

Figure 4.3: The $H^{\infty}$ current control applied to the CBB converter

\subsubsection{Switching logic in order to have the buck or boost mode control}

For the CBB converter to operate in variable input voltage and to have constant output voltage, the switching logic must be applied in order to help the CBB converter to respond to these condition $[43,49,50]$. There are two techniques that can be used in the $\mathrm{CBB}$ converter in order to have different mode of applications such as the buck and the boost operation. The first technique is to use the duty cycle range for the buck or boost operation $[49,52]$. It requires the information of the duty cycle for the whole period of the cycle. In this technique, the control structure needs a dual loop which consists of inner loop and outer loop control. This controller creates more complex mathematical analysis when it is used in the average and linerisation method. 
The second technique, which is the easiest technique, is to use the logic sequence. The AND and OR logic sequences are used to determined the switching signal for the $\mathrm{CBB}$ converter. The logic operations are depend to the input $\left(V_{s}\right)$ and output $\left(V_{o}\right)$ voltage from the CBB converter. It is when the amount of $V_{s}$ is bigger than $V_{o}$ it operates as buck and when $V_{o}$ is bigger than $V_{s}$ the boost is operated. This technique is easier to model compared to the first technique. Due to the simplicity, the second technique is used to control the operation of the the CBB converter. The operations are: when it operates in buck mode the switch at $S_{1}$ follows the PWM signal generated by the current controller while $S_{2}$ is switch off and when the CBB converter operates in boost mode the $S_{1}$ is on while the $S_{2}$ follows the generate signal form the PWM signal. Figure 4.4 shows the arrangement for the logic sequence applied to the CBB converter.

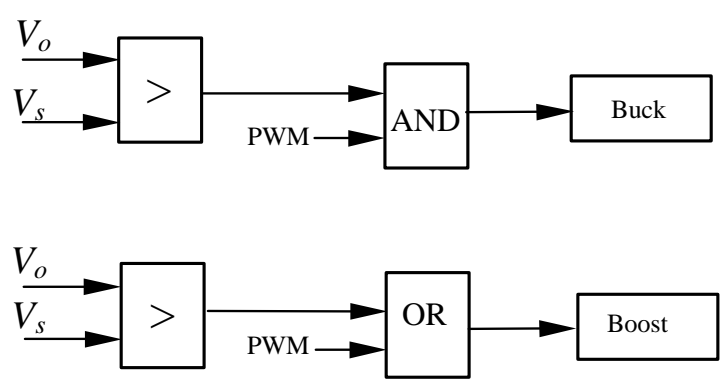

Figure 4.4: The block diagram of logic sequence used in the CBB converter for boost or buck mode operation

\subsection{3 $H^{\infty}$ current controller design}

Generally, there are two ways to model the plant for the $H^{\infty}$ control theory application. In here the plant is the CBB converter. The first method is to use the state-space model that has been presented in [94, 100, 102] for the inverter plant. The second method is to use the transfer function model for the dc-dc converter proposed in $[113,116]$. At the end, both techniques can be used to determine the optimal value for the controller value using $H^{\infty}$ control theory. In this work, the $H^{\infty}$ controller value is given by the stabilising compensator $K$ that is used for the current controller structure. This control structure is shown in Figure 4.5. In this figure, the plant $G$ is developed by the boost converter topology circuit. The disturbance parameters $w$ and the input $u$ are the inputs to the plant. The outputs for the plant are given by $y$ and $e$ where $y=I_{s}$ and $e$ is the error between the input reference current and the input measure current at the CBB converter.

The complete $H^{\infty}$ control structure to the CBB converter plant is shown in Figure 4.6. In this configuration the new regulated output component to the plant has been 


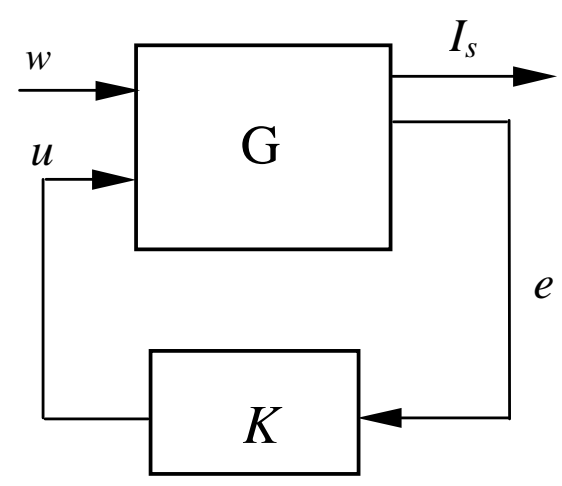

Figure 4.5: The $\mathrm{CBB}$ with the $\mathrm{H}^{\infty}$ control scheme

introduced in order to increase the robustness of the current controller to the CBB converter which are the $z_{1}, z_{2}$ and $z_{3}$. These new outputs are related to the error $e$, the $I_{s}$, and the $u$. All these outputs are multiplied with the weighting functions that are presented by $W_{1}, W_{2}$ and $W_{3}$. The next subsection shows how this new extended plant that consists all the parameters show in Figure 4.6 can be generated in order to calculate the $K$ value using the $H^{\infty}$ control theory.

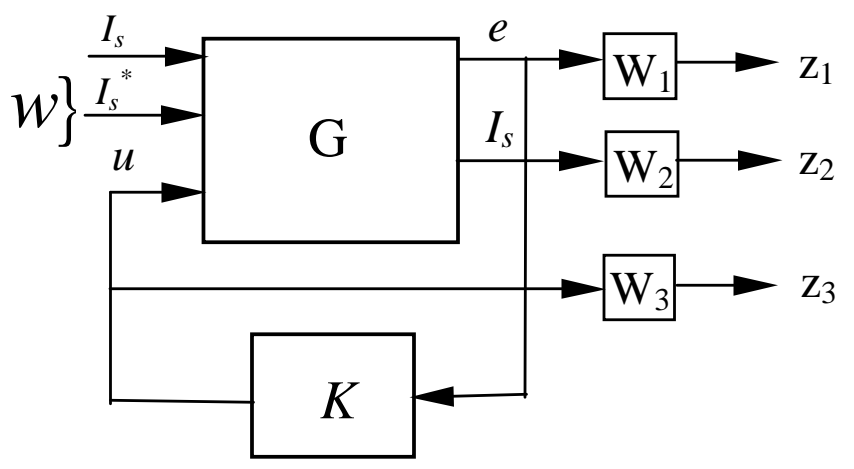

Figure 4.6: The block diagram of the $H^{\infty}$ current control scheme in CBB converter

Before $K$ value can be calculated, the transfer function equations for the CBB converter need to be determined. Using the Kirchhoff's current law (KCL) and Kirchhoff's voltage law (KVL) at boost mode in Figure 4.1 the equations can be written as:

$$
\begin{aligned}
I_{s} & =I_{R}+I_{C_{c b b}}, \\
I_{s}-I_{R} & =s C_{c b b} V_{o}, \\
\frac{1}{s C_{c b b}} & =\frac{V_{o}}{I_{s}-I_{R}},
\end{aligned}
$$


and

$$
V_{o}-V_{s}=\left(s\left(L_{c b b}+R_{c b b}\right)\right) I_{s} .
$$

The $v_{c_{b b}}$ is also known as the output voltage $\left(V_{o}\right)$ at the CBB converter. Equations 4.11 and 4.12 can be modelled in a block diagram where it shows in Figure 4.7 in order to determine the transfer function for the $\mathrm{CBB}$ converter plant.

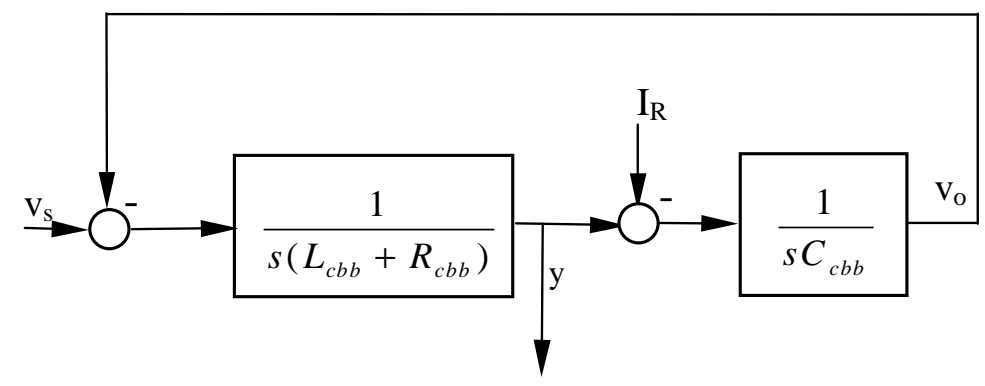

Figure 4.7: The block diagram of the CBB converter

As can be seen from Figure 4.7, the inputs are coming from the $I_{R}$ and $V_{s}$ which are known as the disturbances to the plant. By using the average analysis to the $V_{s}$ at the CBB converter the relationship for the duty cycle $(u)$ can be stated as $V_{o} u=V_{s}$. The final transfer function for the plant can be written as:

$$
\frac{I_{s}}{u}=\frac{V_{o} s C}{s^{2} L_{c b b} C_{c b b}+R_{L_{c b b}} C_{c b b} s+1}, \frac{I_{s}}{I_{R}}=\frac{-1}{s^{2} L_{c b b} C_{c b b}+R_{L_{c b b}} C_{c b b} s+1} .
$$

For the $H^{\infty}$ control application some modifications have been made from Figure 4.7, which includes the weighting functions and the final structure is given in Figure 4.8. From this figure, the new input $I_{s}^{*}$ has been considered in order to calculate the $H^{\infty}$ controller $K$. As known, this structure can be used in state-space matrix notation by changing the transfer function in equation 4.13 to state-space using $t f 2 s s$ command that is available in the MATLAB program.

Equation 4.13 shows the complete transfer function plant for the CBB converter, where the outputs are the input current $\left(I_{s}=y\right)$ and $e$ while the inputs are the $u$ and $I_{R}$. For the $H^{\infty}$ control theory, the new extended plant given by $P$ needs to be modelled by including all the inputs and the weighting functions of $W_{1}, W_{2}$ and $W_{3}$. This extended plant can be modelled using the M-File format in the MATLAB software by changing the transfer functions in equation 4.13 to the state-space matrix that is suitable to determine the $H^{\infty}$ control value.

The state-space matrix calculation is given as described. In the CBB converter, the $I_{s} \simeq I_{L}$ for the inductor and the capacitor voltage $V_{o}$ are the state variables to the plant 


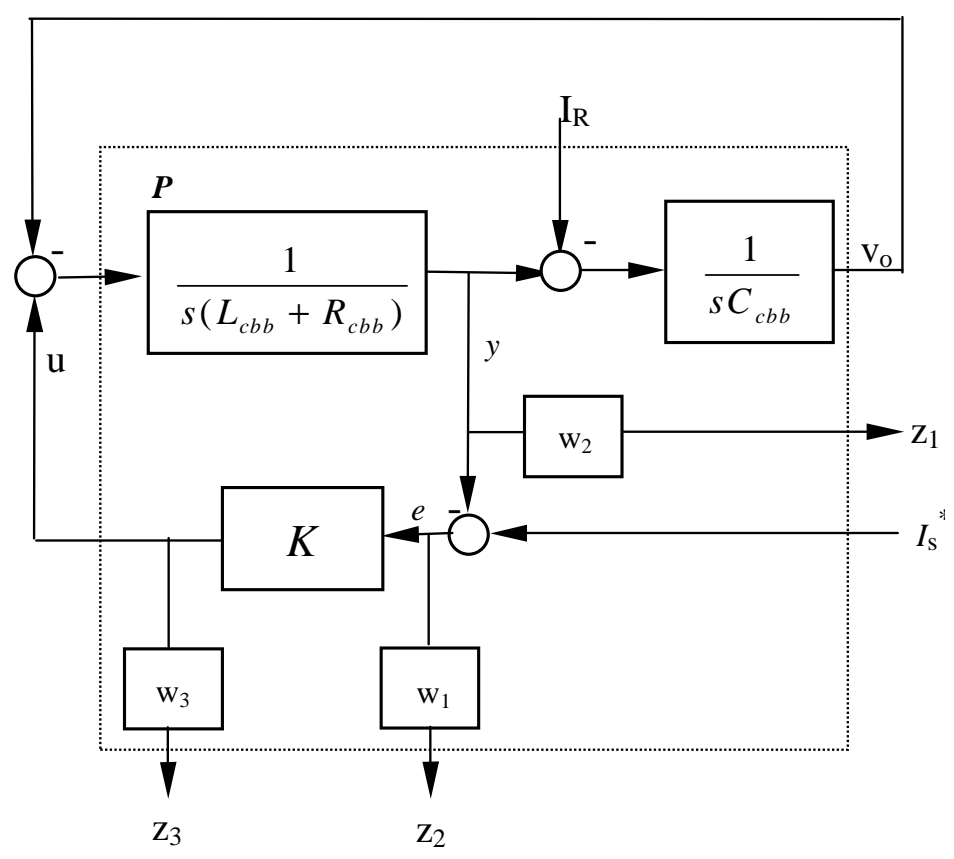

Figure 4.8: The complete structure for CBB converter with the inputs and outputs in $H^{\infty}$ control model

that can be written as:

$$
x=\left[\begin{array}{l}
I_{s} \\
V_{o}
\end{array}\right]
$$

From Figure 4.8, the disturbances $\omega$ and the $u$ can be written as:

$$
\left.\left[\begin{array}{c}
{[w]} \\
u
\end{array}\right]=\left[\begin{array}{c}
I_{R} \\
I_{s}^{*} \\
D
\end{array}\right]\right] .
$$

The state-space equations of the plant can be written as:

$$
\begin{aligned}
& \dot{x}=A x+B_{1} w+B_{2} u \\
& y=C x+D_{1} w+D_{2} u
\end{aligned}
$$

where $y$ is the first output and $A, B_{1}, B_{2}, C$ and $D_{1}$ are the matrix components of the plant. The $G$ with $I_{s}$ output is shown as:

$$
\begin{gathered}
y=I_{s} \\
G_{1}=\left[\begin{array}{c|cc}
A & B_{1} & B_{2} \\
\hline C_{1} & D_{11} & D_{12}
\end{array}\right] .
\end{gathered}
$$


The second output for CBB converter output matrix is shown from Figure 4.8:

$$
y=e=I_{s}^{*}-I_{s} .
$$

From equation 4.14 it can be seen that the reference current needs to be in state-space matrix order. The state-space of the output combined with the $I_{s}^{*}$ is given by:

$$
G_{2}=\left[\begin{array}{c|cc}
A & B_{1} & B_{2} \\
\hline C_{2} & D_{21} & D_{22}
\end{array}\right] .
$$

The complete state-space matrix notation that includes both the outputs is shown as:

$$
G=\left[\begin{array}{c|cc}
A & B_{1} & B_{2} \\
\hline C_{1} & D_{11} & D_{12} \\
C_{2} & D_{21} & D_{22}
\end{array}\right]
$$

As mentioned above, the weighting functions have their own function. The function of $W_{1}$ is for tracking error performance, $W_{2}$ is for weight controller [114] or to have a robustness effect at the output, and $W_{3}$ is used to achieved the full rank condition for the new plant $P$. Here the $W_{1}$ can be developed from the low pass transfer function equation [113] while $W_{2}$ is given from [114] where it is a second order transfer function. The state-space model for these weighting function can be shown in a general formula by:

$$
W_{1}, W_{2}, W_{3}=\left[\begin{array}{c|c}
A_{w_{1,2},} & B_{w_{1,2,3}} \\
\hline C_{w_{1,2,3}} & D_{w_{1,2,3}}
\end{array}\right]
$$

The complete new extended plant $P$ which is shown in Figure 4.8 is represented in the bounding block with the new output vectors of $z_{1}, z_{2}$ and $z_{3}$ can be derived as:

$$
\begin{aligned}
z_{1} & =I_{s} \times W_{1} \\
& =\left[\begin{array}{c|cc}
A & B_{1} & B_{2} \\
\hline C & D_{11} & D_{12}
\end{array}\right]\left[\begin{array}{c|c}
A_{W_{1}} & B_{W_{1}} \\
\hline C_{W_{1}} & D_{W_{1}}
\end{array}\right]\left[\begin{array}{c}
\omega \\
u
\end{array}\right] \\
& =\left[\begin{array}{cc|cc}
A & 0 & B_{1} & B_{2} \\
B_{W_{1}} C_{1} & A_{W 1} & B_{W_{1}} D_{11} & B_{W_{1}} D_{12} \\
\hline D_{W_{1}} C_{1} & C_{W_{1}} & D_{W_{1}} D_{11} & D_{W_{1}} D_{12}
\end{array}\right]\left[\begin{array}{c}
\omega \\
u
\end{array}\right],
\end{aligned}
$$




$$
\begin{aligned}
z_{2} & =e \times W_{2} \\
& =\left[\begin{array}{c|cc}
A & B_{1} & B_{2} \\
\hline C_{2} & D_{21} & D_{22}
\end{array}\right]\left[\begin{array}{c|c}
A_{W_{2}} & B_{W_{2}} \\
\hline C_{W_{2}} & D_{W_{2}}
\end{array}\right]\left[\begin{array}{l}
\omega \\
u
\end{array}\right] \\
& =\left[\begin{array}{cc|cc}
A & 0 & B_{1} & B_{2} \\
B_{W_{2}} C_{2} & A_{W_{2}} & B_{W_{2}} D_{21} & B_{W_{2}} D_{22} \\
\hline D_{W_{2}} C_{2} & C_{W_{2}} & D_{W_{2}} D_{21} & D_{W_{2}} D_{22}
\end{array}\right]\left[\begin{array}{l}
\omega \\
u
\end{array}\right],
\end{aligned}
$$

and

$$
\begin{aligned}
z_{3} & =W_{3} \times u \\
& =\left[\begin{array}{l|ll}
A_{W_{3}} & 0 & B_{W_{3}} \\
\hline C_{W_{3}} & 0 & D_{W_{3}}
\end{array}\right]\left[\begin{array}{l}
\omega \\
u
\end{array}\right] .
\end{aligned}
$$

By combining equations $4.16,4.17$ and 4.18 , the $P$ can be written as:

$$
P=\left[\begin{array}{c|cc}
A_{(\text {new })} & B_{1(\text { new })} & B_{2(\text { new })} \\
\hline C_{1(\text { new })} & D_{11(\text { new })} & D_{12(\text { new })} \\
C_{2(\text { new })} & D_{21(\text { new })} & D_{22(\text { new })}
\end{array}\right],
$$

where,

$$
\begin{aligned}
& A_{(\text {new })}=\left[\begin{array}{cccc}
A & 0 & 0 & 0 \\
B_{W_{1}} C_{1} & A_{W_{1}} & 0 & 0 \\
B_{W_{2}} C_{2} & 0 & A_{W_{2}} & 0 \\
0 & 0 & 0 & A_{W_{3}}
\end{array}\right] \\
& B_{1(\text { new })}=\left[\begin{array}{c}
B_{1} \\
B_{W_{1}} D_{11} \\
B_{W_{2}} D_{21} \\
0
\end{array}\right], B_{2(\text { new })}=\left[\begin{array}{c}
B_{2} \\
B_{W_{1}} D_{12} \\
B_{w W_{2}} D_{22} \\
0
\end{array}\right] \\
& C_{1(\text { new })}=\left[\begin{array}{cccc}
D_{W_{1}} C_{1} & C_{W_{1}} & 0 & 0 \\
D_{W_{2}} C_{2} & 0 & C_{W_{2}} & 0 \\
0 & 0 & 0 & C_{W_{3}}
\end{array}\right], C_{2(\text { new })}=\left[\begin{array}{cccc}
C_{1} & 0 & 0 & 0 \\
C_{2} & 0 & 0 & 0
\end{array}\right] \\
& D_{11(\text { new })}=\left[\begin{array}{c}
D_{W_{1}} D_{11} \\
D_{W_{2}} D_{21} \\
0
\end{array}\right], D_{12(\text { new })}=\left[\begin{array}{c}
D_{W_{1}} D_{12} \\
D_{W_{2}} D_{22} \\
D_{W_{3}}
\end{array}\right] \\
& D_{21(\text { new })}=\left[\begin{array}{c}
D_{11} \\
D_{21}
\end{array}\right], D_{22(\text { new })}=\left[\begin{array}{c}
D_{12} \\
D_{22}
\end{array}\right]
\end{aligned}
$$


and the complete $P$ can be shown as,

$$
P=\left[\begin{array}{cccc|cc}
A & 0 & 0 & 0 & B_{1} & B_{2} \\
B_{W_{1}} C_{1} & A_{W_{1}} & 0 & 0 & B_{W_{1}} D_{11} & B_{W_{1}} D_{12} \\
B_{W_{2}} C_{2} & 0 & A_{W_{2}} & 0 & B_{W_{2}} D_{21} & B_{W_{2}} D_{22} \\
0 & 0 & 0 & A_{W_{3}} & 0 & B_{W_{3}} \\
\hline D_{W_{1}} C_{1} & C_{W_{1}} & 0 & 0 & D_{W_{1}} D_{11} & D_{W_{1}} D_{12} \\
D_{W_{2}} C_{2} & 0 & C_{W_{2}} & 0 & D_{W_{2}} D_{21} & D_{W_{2}} D_{22} \\
0 & 0 & 0 & C_{W_{3}} & 0 & D_{W_{3}} \\
\hline C_{1} & 0 & 0 & 0 & D_{11} & D_{12} \\
C_{2} & 0 & 0 & 0 & D_{21} & D_{22}
\end{array}\right] .
$$

For the $H^{\infty}$ controller design, the value for the weighting functions are given by equation 4.20 .

$$
W_{1}=\frac{s+10}{s+1000}, W_{2}=\frac{10}{s+0.01 s+1000}, W_{3}=\frac{0.000205 s+50000}{s+5000} .
$$

By substituting these values into the extended plant, the controller value can be determined in equation 4.21. The M-File program in the MATLAB software can be used in order to calculate this value. The hinfsys command function that has been used in order to generate current controller and it shows as:

$$
H^{\infty}=\frac{-497.1888(s+1.73 e 007)(s+5000)(s+999)\left(s^{2}+0.1008 s+1028\right)}{(s+1.422 e 009)(s+4879)(s+636.9)(s+375.3)\left(s^{2}+0.03133 s+1000\right)} .
$$

Equation 4.21 shows that the $K$ compensator value that consists of very far poles. According to $[21,89]$, these poles cannot be realised by any means. To deal with it, the poles and zeros that are closed to each other can cancel each other, such that the resulting controller is given by:

$$
K=H^{\infty}=\frac{-497(s+999)}{(s+636.9)(s+375.3)} .
$$

\subsubsection{Determining the stability performance for the $H^{\infty}$ current con- troller}

After the $K$ value for controller has been determined, the stability performance that correspond to the $K$ and the $G$ need to be checked. In general feedback control technique, the stability can be checked using the root locus technique. Equation 4.22 shows the poles and zeros are located at the left side of the root locus axis and these poles and zeros are in stable.

For the $H^{\infty}$ control theory, the stability is determined by minimising the closed loop system from the $y$ to $u$ or $T_{y u}$ in $H^{\infty}$ norm functions. It shows that this function 
is related to the specific weighting function shows in Figure 4.8 to each new regulated output. Before the stability can be calculated, the singular values analysis is done to synthesise the controller with the weighting functions using mixed-sensitivity approach $[114,134]$. Singular values analysis is used to show the magnitude (db) of the $W_{1}$ and $W_{2}$ which are corresponded with the sensitivity function $(S)$ and the complementary sensitivity function $(T)$ at the fundamental frequency. This relation can be written as:

$$
\left\|\begin{array}{l}
W_{2} \times T \\
W_{1} \times S
\end{array}\right\|<\left[\begin{array}{l}
1 \\
1
\end{array}\right]
$$

where,

$$
\begin{aligned}
S & =\frac{1}{1+K(s) \times G} \\
T & =1-S
\end{aligned}
$$

By plotting the singular values bode diagram for $W_{2}$ and $T$, the response to each other can be determined where $T$ is related to $\frac{\gamma * G}{W_{2}}$. This technique is called loop shaping, and is used to select the weighting function value for $W_{2}$ in the $H^{\infty}$ control theory. According to [116], the $T$ can be found by making the closed loop transfer function small at high frequencies where $T=1-S$ small as follow $f \rightarrow \infty$. Figure 4.9 shows, the singular values analysis for the $W_{2}$. It shows that, $W_{2}$ is in shape with $T$ but gives small magnitude (db) when $f \rightarrow \infty$ for the T (TT in Figure 4.9) .

After the weighting functions have been selected, the $H^{\infty}$ controller performance can be measured by referring to the closed loop stability value where it is the transfer function from $y$ to the $u$ shown in Figure 2.14. To have a stable condition the value must satisfy the condition given in equation 2.15. By using equation 2.14 as given in the chapter 2, the generated $\left\|T_{y u}\right\|$ and the $\gamma$ from equation 4.19 are given by,

$$
\gamma=104.0387,\left\|T_{y u}\right\|=52.4615
$$

It shows that the stability condition in equation 2.15 has been achieved and makes the $K$ is in stable condition. 


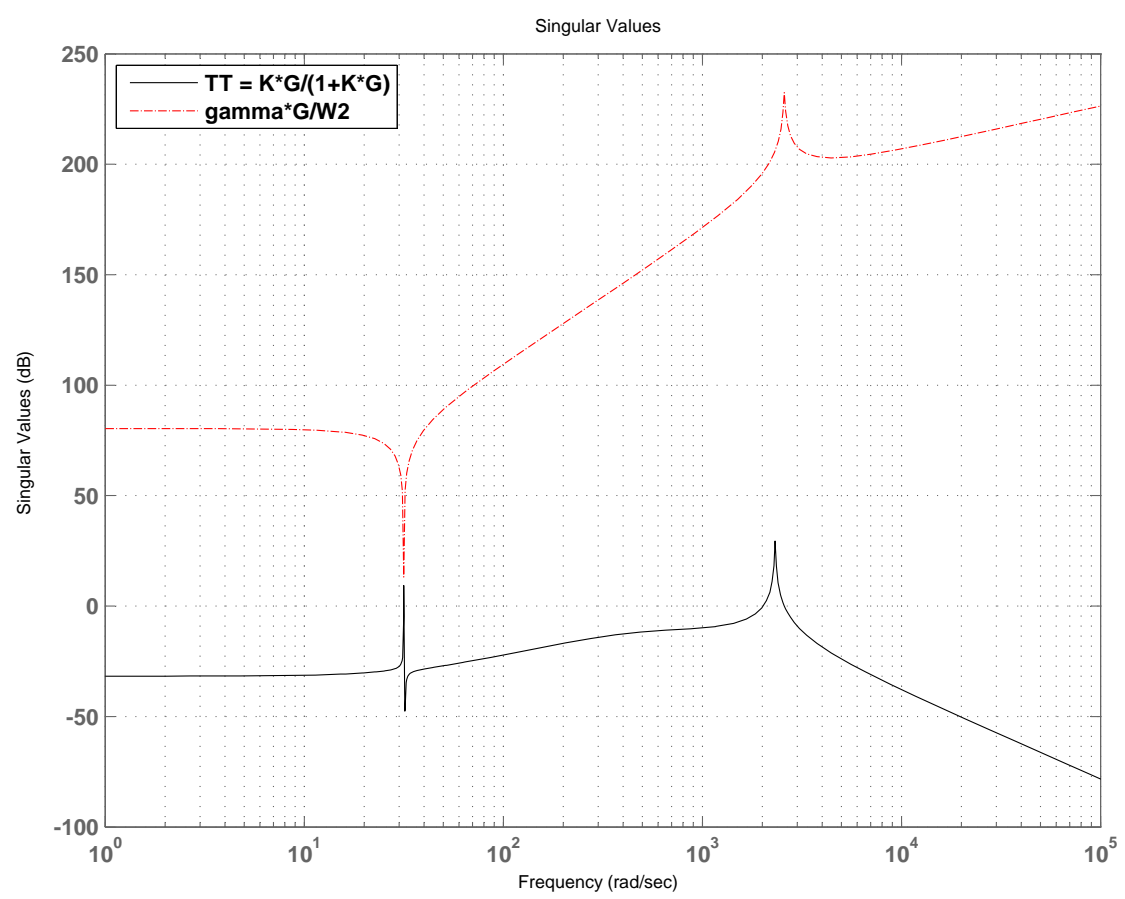

Figure 4.9: The singular analysis bode diagram

\subsection{Simulation results}

In this section, the simulation results for the $\mathrm{CBB}$ converter are shown when it uses to control the input current. The parameters for the CBB converter are given in Table 4.1 .

Table 4.1: Parameters of the CBB converter

\begin{tabular}{|c|c|}
\hline Parameters & Value \\
\hline \hline$L_{c b b}$ & $300 \mathrm{uH}$ \\
\hline$C_{c b b}$ & $500 \mathrm{uF}$ \\
\hline$R_{L_{c b b}}$ & $0.01 \Omega$ \\
\hline
\end{tabular}

The simulations were carried out by using MATLAB Simulink 8 with the maximum step size of 0.01 miliseconds. The same simulation time was used with the LG part in the energy conversion section. For the $H^{\infty}$ current control, the target for the current is set to $20 \mathrm{~A}\left(I_{s}^{*}\right)$. This value has been set because of the calculated rated value for the input current which is given in equation 4.8. For the simulation results two categories of outputs were collected. The first outputs were collected from LG outputs of the energy conversion which is shown in Figure 4.10, and the second outputs are form CBB converter operation with the current control strategy shown in Figure 4.11. The reason why these outputs are shown here is because by controlling the CBB converter, the outputs at LG of the energy conversion can be controlled too. 
Figure 4.10a shows the output force has been maintained at the constant value along the landing process which has been calculated from equation 4.5. This means that this force will not effect the comfort of the passengers during energy conversion. This force is related to the deceleration speed and it has been controlled which is the target for the $\mathrm{CBB}$ converter and it has been calculated as in equation 4.4. The deceleration output is shown in Figure 4.10b. For the power generated at the LG section, the profile has been changed from exponential decay shows in Figure 3.11 to linearly decreasing power as shown Figure 4.10c. By having this power profile, suitable energy storage can easily be modelled and controlled. All the simulations time is finish at the same time with the power generated graph, which indicates that no additional power has been created during the landing process.

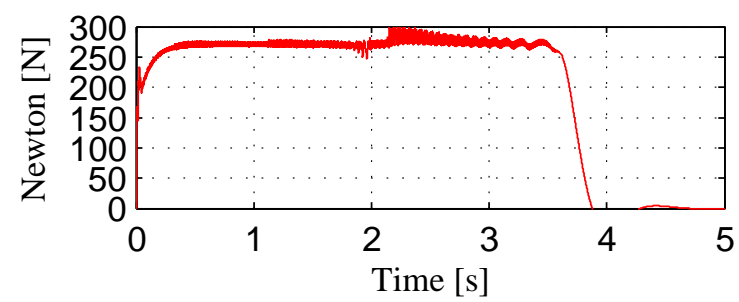

(a) force of the LG

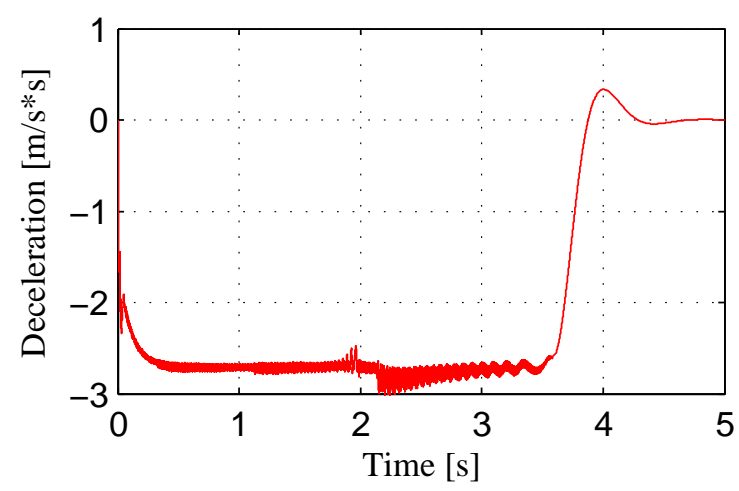

(b) deceleration of the LG

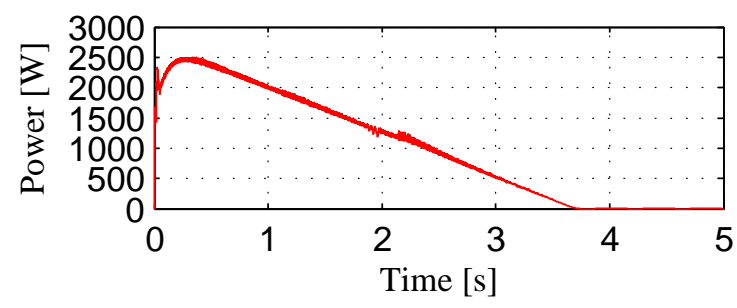

(c) output power of the LG

Figure 4.10: Simulation results at LG output

For the CBB converter outputs, the $H^{\infty}$ current control was used and the results are shown in Figure 4.11. For Figure 4.11a the current is maintained at the target value of $20 \mathrm{~A}$. It shows that the current controller is working properly regarding the variable 


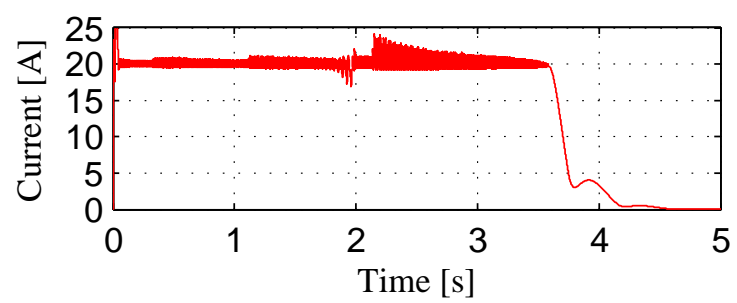

(a) input current of the CBB converter

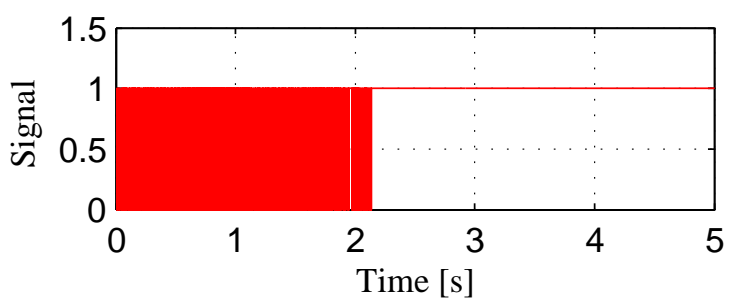

(b) buck signal at $\mathrm{CBB}$ converter when $\left(V_{o}>V_{s}\right)$

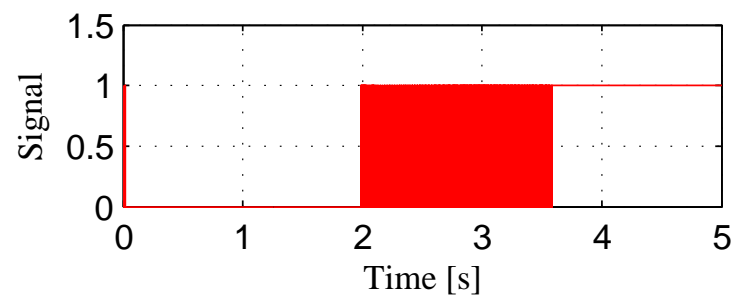

(c) boost signal at CBB converter when $\left(V_{o}<V_{s}\right)$

Figure 4.11: Simulation results when $H^{\infty}$ current control applied to the CBB converter magnitude current at the input of the CBB converter due to decreasing current from the uncontrolled diode rectifier. Figures $4.11 \mathrm{~b}$ and $4.11 \mathrm{c}$ show the switching logic that was happen in the CBB converter regarding the input voltage and the output voltage at the CBB converter. It shows that, this CBB converter can be operated in two modes of operation in order to control the current flow to the load.

Figure 4.12 shows the current error between the $I_{s}^{*}-I_{s}$. The small different is achieved between these two inputs. After $4 \mathrm{~s}$ the value of tracking error is increase and it reaches to the target value $20 \mathrm{~A}$ because there is no $I_{s}$ flowing in the CBB converter due to no generated power from the LG. It explains that the $H^{\infty}$ controller is managed to minimise the tracking error of the input current to the CBB converter. 


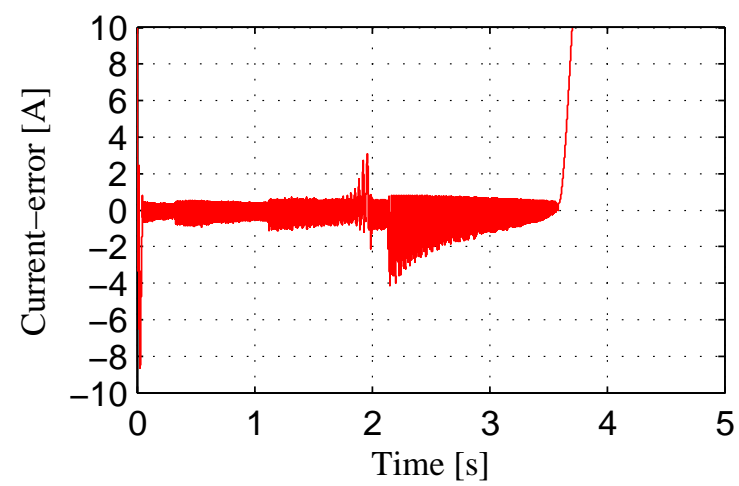

Figure 4.12: The tracking error current $\left(I_{s}^{*}-I_{s}\right)$

\subsection{Concluding remarks}

To control the CBB converter where it is the main part for the overall process in energy conversion. Here, a current control strategy has been applied to the CBB converter, which at the same time controls the outputs from the LG such as the generated force and the power. This is due to the relationship between the force at the LG and the input current of the $\mathrm{CBB}$ converter itself. In this part, the CBB converter have been used, which has the ability to respond to the wide input voltage range but able to maintain the output voltage at the end. The technique called $H^{\infty}$ control theory has been used to the current controller due to its advantage that gives good performance in tracking error and at the same time response to any disturbances and perturbations happen to the $\mathrm{CBB}$ converter. Due to the wide input voltage range, the logic sequence has been proposed in order to operate the $\mathrm{CBB}$ converter operates as either the buck converter at one time or to operate as a boost converter at other times. The generated signals to the switches are based on the PWM signal of the current control, but the sequence is determined by the input and output voltage at the CBB converter. As shown by the results, by controlling the input current for the CBB converter the force can be maintained, the power generated has changed to linearly decrease power and also the deceleration is maintained that gives significant improvement to the comfort of passenger in the aircraft. This will ensure the average power and the maximum power can be calculated in order to determine the size of the energy storage component that will be used to store the excessive energy during landing, and to release the energy when the required power at the load is more than the average power generated from the energy conversion. 


\section{Chapter 5}

\section{Energy storage: The dc-dc converters control for energy storage}

In this chapter, the energy storage element is used in order to temporarily store the excessive energy generated at the beginning of the landing and discharge it when the source power is lower than the required power at the load. This section is known as the energy storage. Two dc-dc converters are used to achieve these conditions; first is to control the dc-link voltage with the energy storage element using the bidirectional converter, second is to increase the dc-link voltage using the boost converter from $60 \mathrm{~V}$ to $300 \mathrm{~V}$, which will be used in the energy transfer section. Ultracapacitor is selected as the energy storage device in order to store the energy from the generated power during landing. At the end of this chapter the simulations processes were conducted by combining the energy conversion with the energy storage to determine the effectiveness of the ultracapacitor in order to respond to the changing resistive load where it determines the required power at the load.

\subsection{Modelling and control of the dc-dc converters for the energy storage}

Figure 5.1 shows the arrangement of the energy storage part that consists of the ultracapacitor, the bidirectional converter and the boost converter. The bidirectional converter consists of two switches, which are $S_{1}$ and $S_{2}$, where the switching signals that coming to them are complementary to each other. The $L_{b d}$ is the inductor for the bidirectional converter, which allows the current to flow in/out the ultracapacitor. This part is connected in parallel to the dc-link voltage in order to maintain the dc-link voltage with the energy conversion connection [64, 65]. 


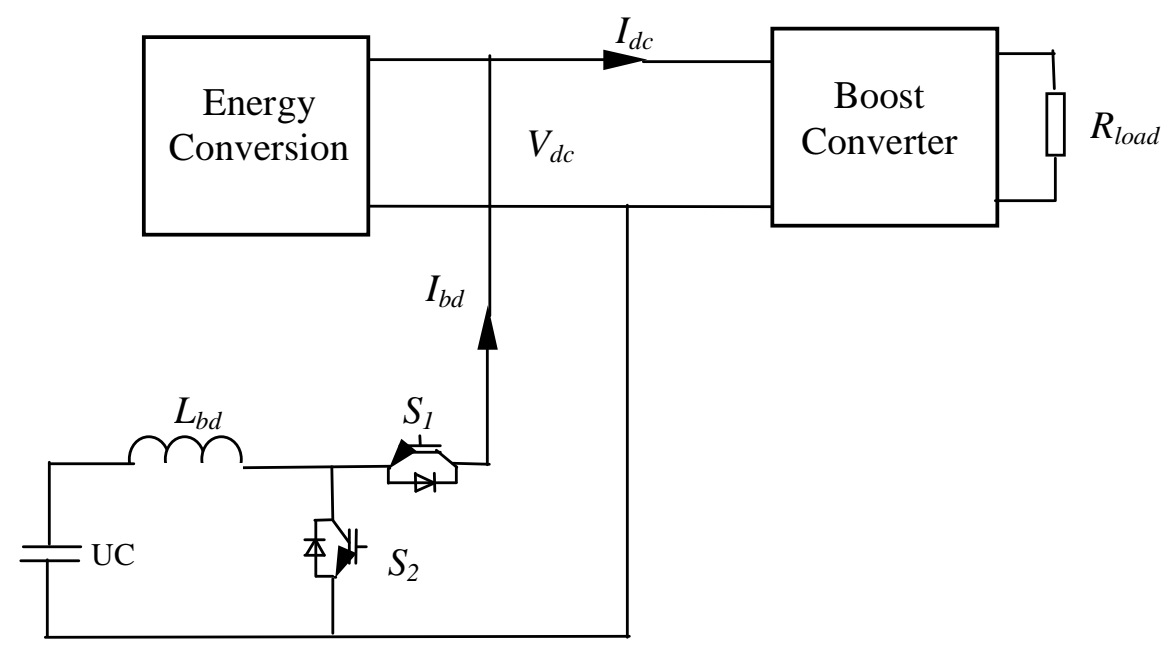

Figure 5.1: The block diagram of the energy storage in an ERLA system

The boost converter is used to increase the dc-link voltage $V_{d c}$ for the next process. The suggested control strategy for the bidirectional converter is the voltage controller at the dc-link. It is because to maintain the dc-link voltage between the energy conversion and the energy storage. The control strategy for the boost converter is by using the voltage controller in order to increase the dc-link voltage to the suitable voltage that will be used for the energy transfer.

\subsubsection{The sizing of the ultracapacitor}

The ultracapacitor has been selected for the ERLA system as the energy storage element. Ultracapacitors have been widely used in energy recovery applications especially when there is braking from vehicles $[25,70,135,136]$. Normally, this ultracapacitor can be connected directly to the dc-link structure, but it will create a problem when the load power is changing. It also can cause the rush current to flow to the ultracapacitor and cause damage to the ultracapacitor. To avoid this situation, the bidirectional converter is used to allow the power flow at the ultracapacitor.

To calculate the ultracapacitor value, the energy stored in the ultracapacitor needs to be determined, it is based on the maximum power generated from the main source [137]. For this case, the maximum power is observed during the landing where it can be analyzed at the energy conversion part. The power simulation data result from chapter 3 has been used in order to determine the ultracapacitor value. The peak power $P_{\text {peak }}$ that has been collected is $2500 \mathrm{~W}$ and with the peak time given from the CBB converter switching frequency is used to determine the ultracapacitor value. The energy during 
the maximum power is used to calculate the ultracapacitor is given as:

$$
E=P_{\text {peak }} \times T_{\text {peak }}
$$

Equation 5.1, shows that the maximum energy that can be stored by the ultracapacitor with referring to the peak power shows in Figure 4.10c. The ultracapacitor size has been calculated based on the equation below:

$$
\begin{aligned}
C_{u c} & =\frac{2 \times P_{\text {peak }} \times T_{\text {peak }} \times 3600}{V_{d c}^{2}} \\
& =\frac{2 \times 2500 \times 0.1 \mathrm{~ms} \times 3600}{3600} \\
& =0.5 \mathrm{~F} .
\end{aligned}
$$

The inductor $L_{b b}$ for the bidirectional converter can be calculated based on the ripple current that flows in and out from the bidirectional converter which is $20 \mathrm{~A}$, which is the same as the controlled current in the CBB converter. The value for the inductor in the bidirectional converter can be calculated by:

$$
\begin{aligned}
\Delta_{l} & =\frac{V_{d c}}{4 \times f_{s w} \times L_{b d}} \\
L_{b d} & =750 \mathrm{uH}
\end{aligned}
$$

where the $\triangle i_{l}$ is known as the ripple current of the flowing current at the bidirectional converter. It is given by $10 \%$ of the rated current and the $f_{s w}$ is the switching frequency $(10 \mathrm{kHz})$ of the converter.

\subsubsection{Control strategy for the bidirectional converter with the ultra- capacitor}

Figure 5.2 shows the arrangement of the dc-link voltage controller for the bidirectional converter. Here, the ultracapacitor voltage is not been controlled in order to allow the capacitor to store more energy if the amount of generated power is changing due to different speed and mass of the aircraft have been used. The PI control method has been selected as a control strategy to generate the PWM signal for the switching signals. This controller is easier to design by applying the trial and error method. Two switches, $S_{1}$ and $S_{2}$ that are complementary to each other are suitable for this application, as stated in $[57,67,78]$. 


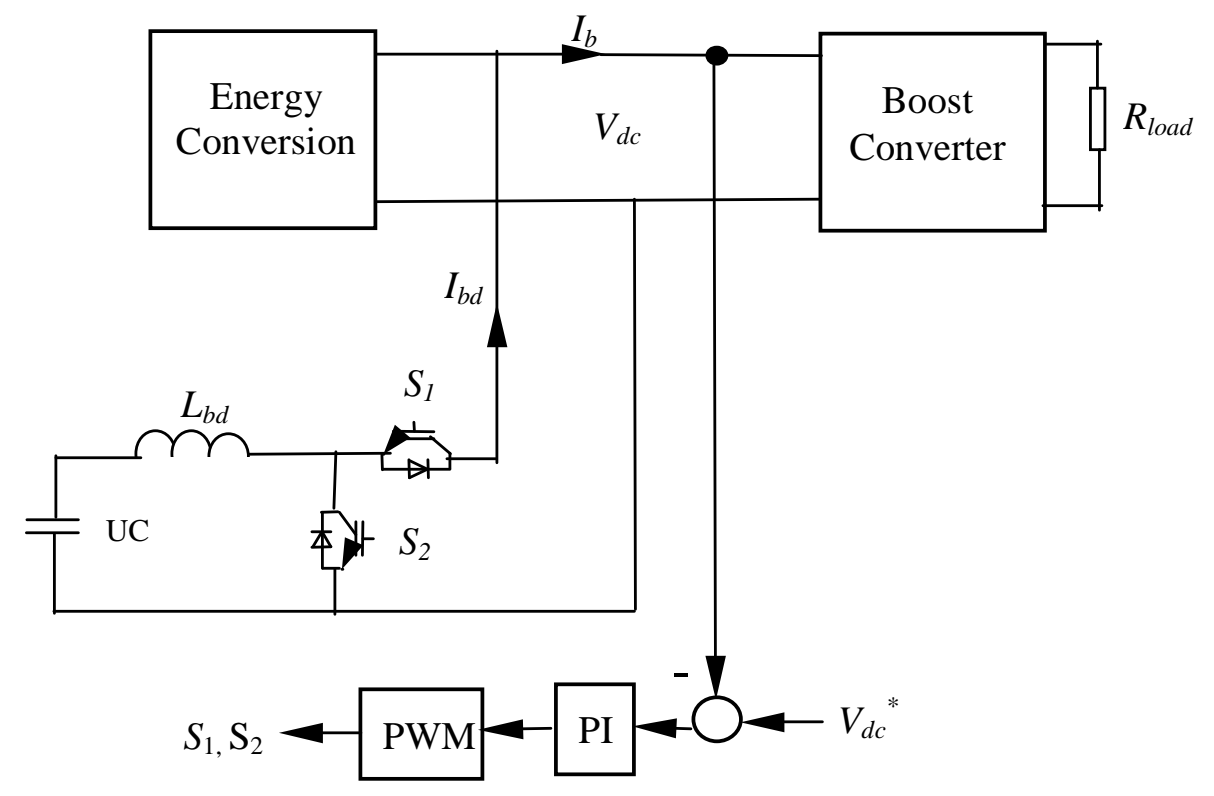

Figure 5.2: The bidirectional converter with voltage control at the dc-link

For the ultracapacitor response, two modes of operation in order to allow the energy flows are described as:

- The charging mode

This happens when the load power is lower than the generated power at the LG. This different power will be stored at the ultracapacitor in order to be used during energy transfer. Here, the positive current flows because the current is coming from the dc-link to the ultracapacitor. This current has not been controlled in order to allow the amount of current following is depended to the amount of power from energy conversion. Due to this condition, the voltage at the ultracapacitor is increased to the rated value.

- The discharging mode

The discharging mode happens when the load power is greater than the source power. In this condition, the ultracapacitor will release the energy that has been stored before. The time taken to release all the energy depends on the amount of current flow to the dclink and it response also to the load power. The dc-link voltage needs to be maintained in order to make sure that continuous voltage can be supplied to the inverter. 


\subsection{Modelling and control of the boost converter}

The last dc signal before it can be transferred to the grid is to boost up the input voltage by using the boost converter that is shown in Figure 5.3. The output of the boost converter is $300 \mathrm{~V}$ which is a suitable to generate around $170 V_{L L}$ (line to line voltage) when it is connected to the grid. This output voltage will be connected to the grid using the inverter to make sure it can transfer the energy that has been stored in the ultracapacitor and from the energy conversion source to the electrical grid or load.

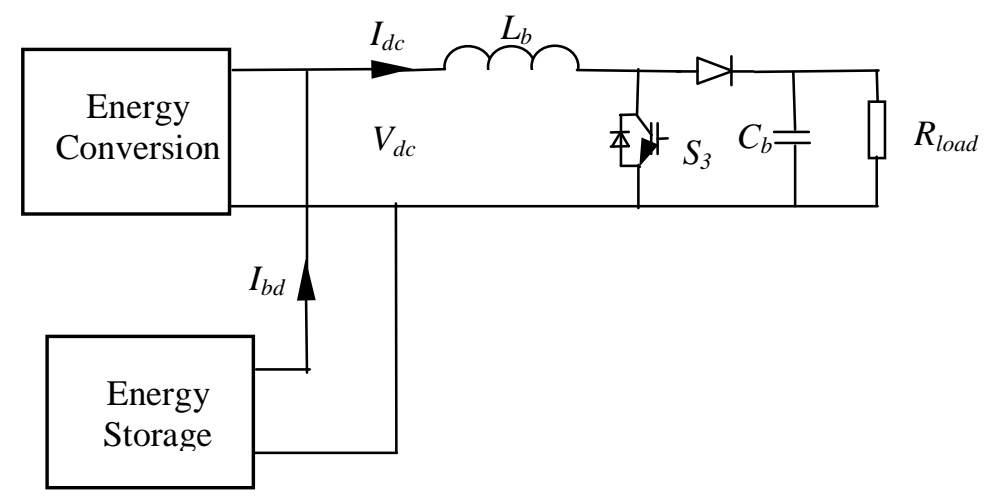

Figure 5.3: The boost converter circuit in energy storage

The parameters for the boost component can be calculated using the general calculation of the boost converter given in equation 5.4. The inductor is calculated using:

$$
L_{b} \geqslant L_{\min }=\frac{D(1-D)^{2}}{2 \times f} \times \frac{\left(V_{\text {rms }}\right)^{2}}{P_{\text {rated }}}
$$

where the $V_{r m s}$ is the output voltage from the inverter, $P_{\text {rated }}$ is the rated value for the power inverter, $D$ is the duty cycle of the boost converter and $f$ is the switching frequency. The given values for these parameters are shown in Table 5.1.

Table 5.1: Parameters to calculate the inductance and capacitance at boost converter

\begin{tabular}{|c|c|}
\hline Parameters & value \\
\hline \hline$V_{\text {rms }}$ & $98 \mathrm{~V}$ \\
\hline$P_{\text {rated }}$ & $1000 \mathrm{~W}$ \\
\hline$f$ & $10 \mathrm{kHz}$ \\
\hline$D$ & 0.8 \\
\hline
\end{tabular}

The minimum value for the inductor using equation 5.4 is given by:

$$
L_{\text {min }}=15.36 \mathrm{uH}
$$


Here, the inductance for the $L_{b}$ is selected as $250 \mathrm{uH}$. For the capacitor capacitance, the $C_{b}$ can be calculated based to equation :

$$
r=\frac{D}{\frac{\left(V_{\text {rms }}\right)^{2}}{P_{\text {rated }}} \times C_{b} \times f}
$$

where $r$ is the voltage ripple and it is must less then $5 \%$. The calculated $C_{b}$ is about $166.59 \mathrm{uF}$. The value of $350 \mathrm{uF}$ has been used for the simulation.

At the load voltage, the voltage is maintained at $300 \mathrm{~V}$ while the power is determined by the resistive load. By changing the resistive load value, the power absorb by the load is also changing. It means that, if the load value is higher, the power absorb to the load is lower and vice versa when the resistive is low. Generally, this will affect the current flows from the bidirectional converter, as will be shown in the simulation results.

\subsubsection{Control strategy for boost converter}

For boost converter control, the standard PI controller has been used. This controller has been applied to this converter because of the maturity of the controller itself, and it is easy to tune the $K_{p}$ and $K_{i}$ parameters to achieve the control target. It is to increase the input voltage that coming from the dc-link to the target value $V_{\text {load }}^{*}$. Here, the controller will maintain the duty cycle that is needed for the switching. Figure 5.4 shows the arrangement of the PI control at the boost converter.

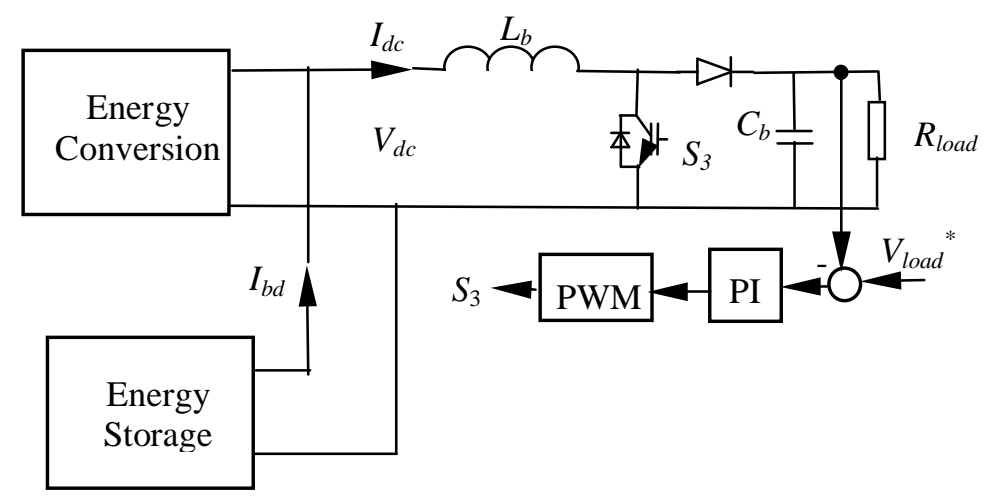

Figure 5.4: The voltage control for boost converter

The general PI control is given by:

$$
P I(s)=K_{p}+\frac{K_{i}}{s}
$$

where $K_{p}$ is the proportional gain and $K_{i}$ is the integral gain. 


\subsection{Simulation results}

The evaluation of the proposed energy storage part is combined with the energy conversion part are used in order to determine the effectiveness of the proposed control strategy. For this analysis, two simulations were carried out with different resistive loads value that represent the different power at the load. With different powers, the time taken for the ultracapacitor to release the energy to the load will be different. The simulation parameters for the energy storage part are given in Table 5.2.

The simulations were conducted in the MATLAB Simulink 8.0 with a step size of 0.01 miliseconds. The time taken for the simulation is from $t=0 \mathrm{~s}$ to $t=6 \mathrm{~s}$. For the dc-link voltage the target is to maintain the voltage at $60 \mathrm{~V}$. This voltage is low, and it needs to increase to a reasonable output voltage at the boost converter output, where the target set voltage is $300 \mathrm{~V}$. This voltage is more suitable for use in energy transfer the ERLA process.

Table 5.2: Parameters value for the energy storage

\begin{tabular}{|c|c|c|c|}
\hline Parameters & Value & Parameters & Value \\
\hline \hline$L_{b d}$ & $150 u \mathrm{H}$ & $L_{b}$ & $250 u \mathrm{H}$ \\
\hline$U C$ & $0.5 \mathrm{~F}$ & $C_{b}$ & $350 u F$ \\
\hline
\end{tabular}

\subsubsection{With the $R_{\text {load }}=100 \Omega$ at the load}

The system responses to the $100 \Omega$ load are shown in Figures 5.5, 5.6 and 5.7. Figure 5.5 shows the outputs at the energy conversion part. From these outputs, the generated power and the deceleration graphs are still the same due to the unaffected current control at the CBB converter. Figure 5.5c, shows the current at the $L_{c b b}$. The reason why this current was measured is to estimate the rated value of the current that will flow to the ultracapacitor. This explained that, by adding the energy storage part, there is no effect on the energy conversion part. 


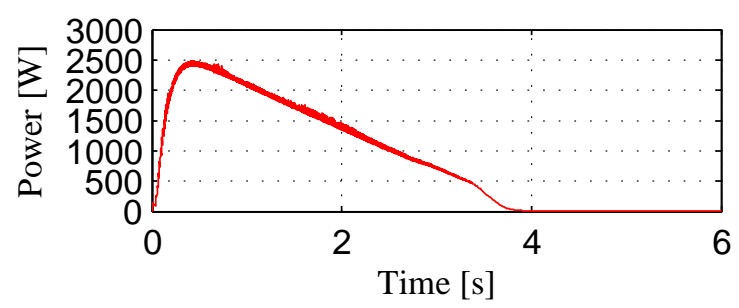

(a) power generated during landing

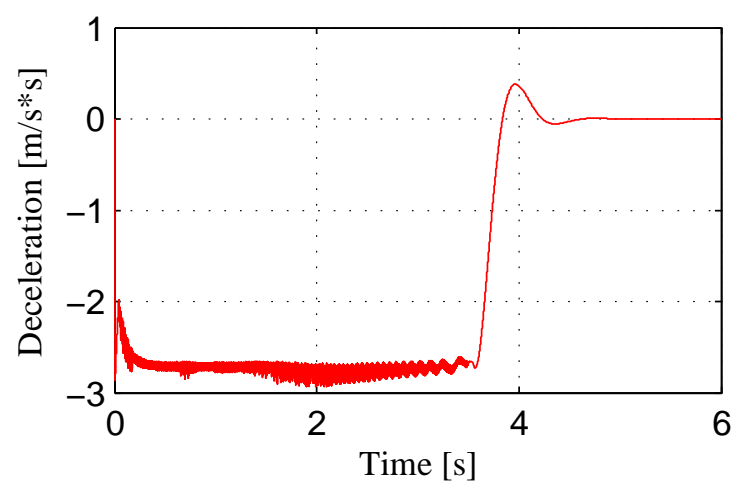

(b) deceleration speed during landing

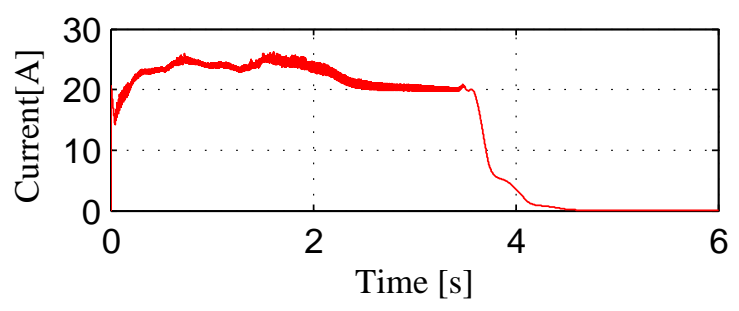

(c) inductor current at CBB converter

Figure 5.5: Simulation results at energy conversion outputs

Figure 5.6 shows the outputs at the energy storage part which includes the bidirectional converter and the boost converter with the proposed controller. Here, the dc-link voltage has been maintained at the target value of $60 \mathrm{~V}$ which is shown in Figure 5.6a with using the bidirectional converter. Figure 5.6b shows the output at the boost converter that has been maintained to the target voltage ready for the next energy transfer process. Figure 5.6c shows the rated current at the load. The time taken for both voltages to reach zero are the same time with the power generated at the LG side shows in Figure 5.5a. 


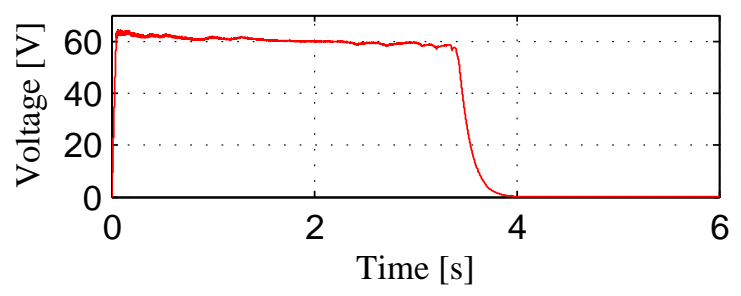

(a) dc link voltage between the energy conversion and energy storage

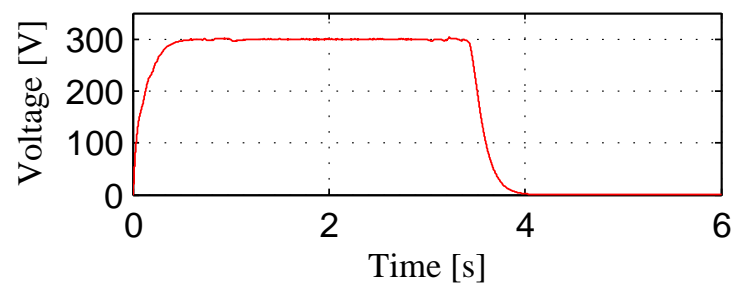

(b) output load voltage at boost converter

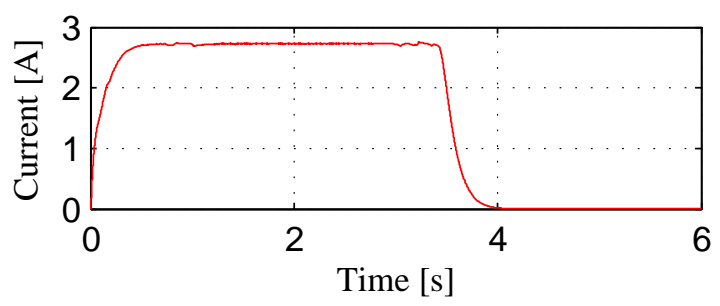

(c) output load current

Figure 5.6: Simulation results at the energy storage part $\left(R_{\text {load }}=100 \Omega\right)$

Figure 5.7 shows the outputs from the ultracapacitor. From these results, the direction of the current flowing is changing from positive current to negative current in order to absorb or to release the energy from the ultracapacitor. This magnitude of current must be lower than the rated value of $20 \mathrm{~A}$. The ultracapacitor voltage output shows, voltage response due to charging and discharging of the energy from the ultracapacitor to the load. 


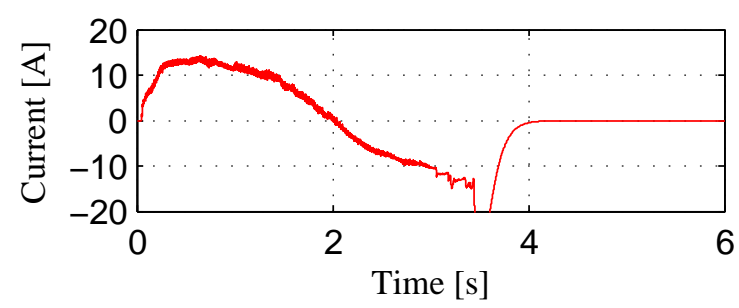

(a) ultracapacitor current during absorbing and releasing the energy

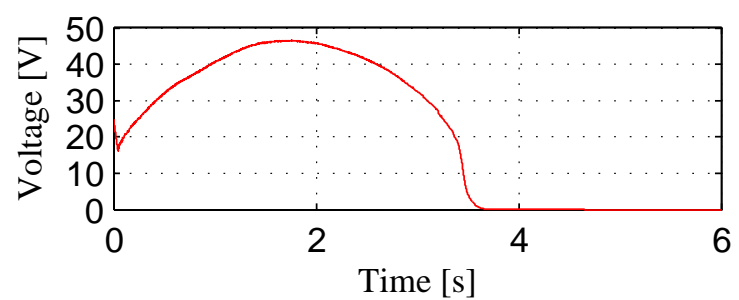

(b) ultracapacitor voltage during absorbing and releasing the energy

Figure 5.7: The ultracapacitor outputs when the load is $R_{\text {Load }}=100 \Omega$

\subsubsection{With the $R_{\text {load }}=150 \Omega$ at the load}

The simulation was run again, but this time the load was changed from $100 \Omega$ to $150 \Omega$. By changing the load, the power transferred to the load is also changed because the power is inversely proportional to the resistive loads value. Figures 5.8, 5.9 and 5.10 show the results that are exactly the same with the results form $R_{\text {load }}=100 \Omega$.

Figure 5.8 shows the outputs at the energy conversion part. It shows that, the deceleration and speed time are finish at the same time when the generated power is zero. It indicates that by changing the load the outputs form the energy conversion will not been effected. From Figures 5.9 and 5.10 its show that the outputs at the energy storage can be maintained at the target voltage, but the difference is the time taken to maintain the voltage for the dc-link voltage and the load voltage are more longer compared to Figure 5.6. This is because, less power is needed from the load by referring to $P_{\text {load }}=\frac{V_{\text {load }}^{2}}{R_{\text {load }}}$ and at the same time the energy that has been stored has longer release time. For this reason, the time taken to release the energy from the ultracapacitor to the load is more than $4 \mathrm{~s}$ as shown in Figure 5.10 due to low current flows through the bidirectional converter compared to Figure 5.7a. 


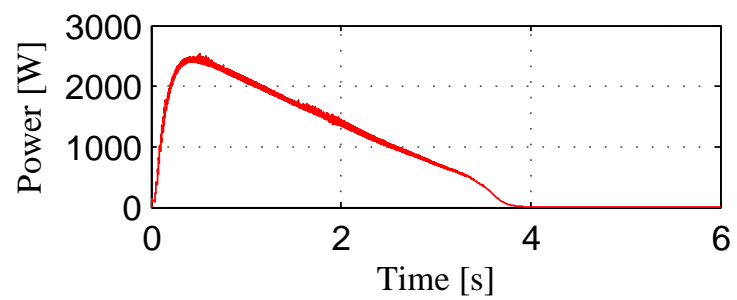

(a) power generated during landing

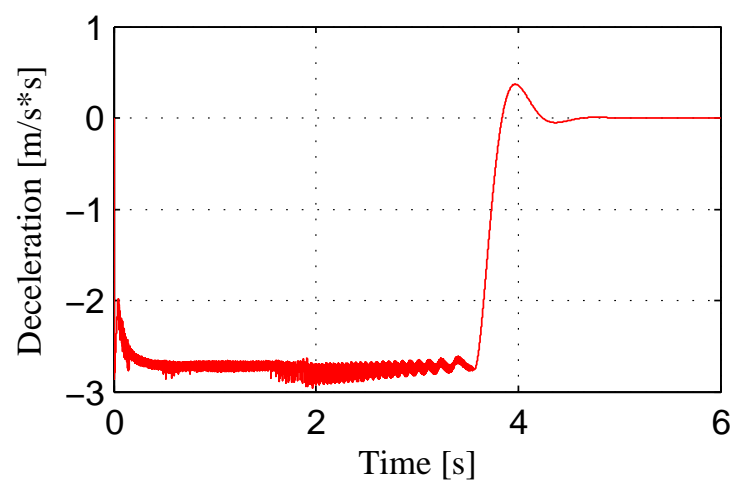

(b) deceleration speed during landing

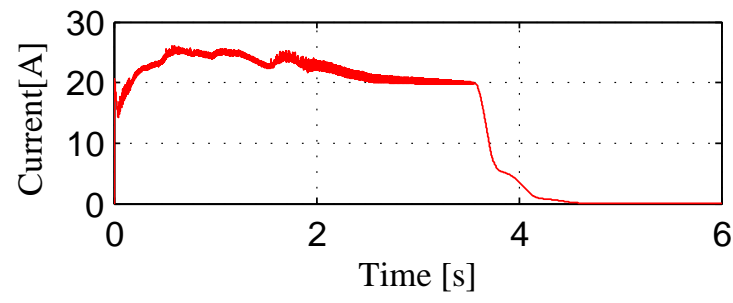

(c) inductor current at CBB converter

Figure 5.8: Simulation results at energy conversion outputs 


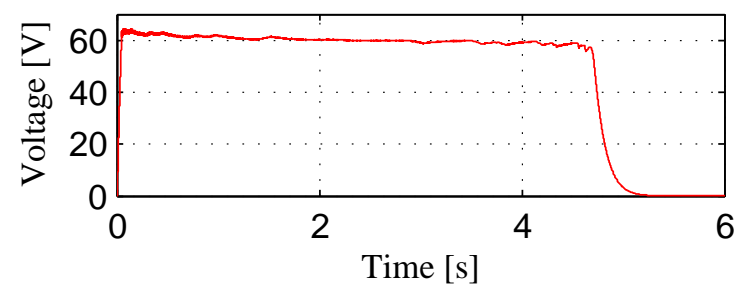

(a) dc link voltage between the energy conversion and energy storage

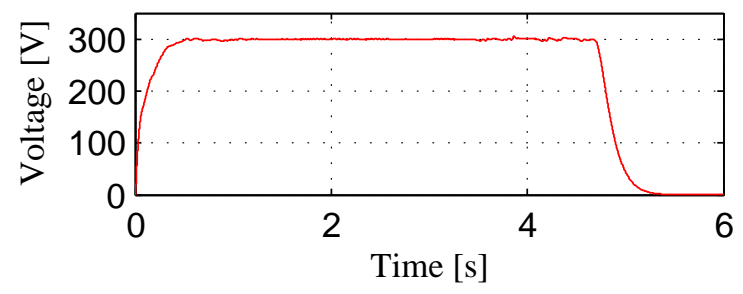

(b) output load voltage at boost converter

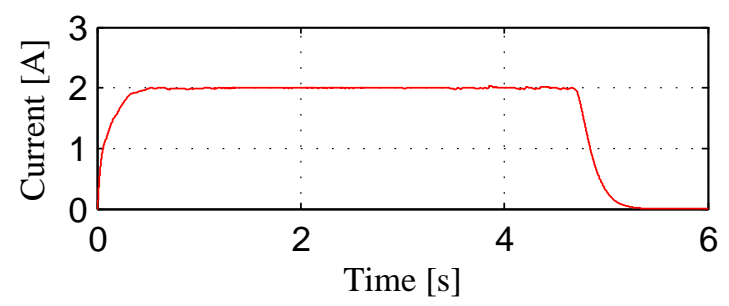

(c) output load current

Figure 5.9: Simulation results at the energy storage part $\left(R_{\text {load }}=150 \Omega\right)$

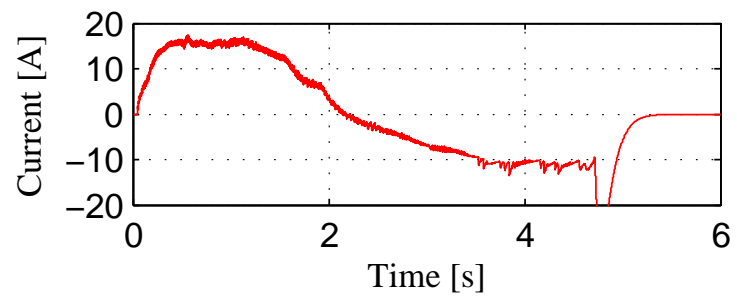

(a) ultracapacitor current during absorbing and releasing the energy

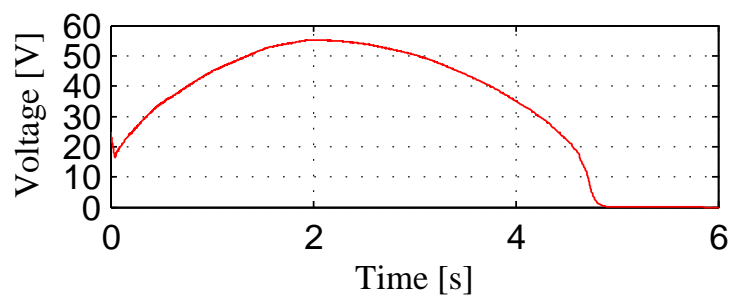

(b) ultracapacitor voltage during absorbing and releasing the energy

Figure 5.10: The ultracapacitor outputs when the is $R_{\text {Load }}=150 \Omega$ 


\subsection{Concluding remarks}

As a conclusion to this chapter, the ultracapacitor can be used as a storage element in the ERLA application. The voltage at the ultracapacitor has not been controlled in order to give freedom to the ultracapacitor to store the energy regarding to the energy generated from the source. With the bidirectional converter connected to the ultracapacitor, the proposed control strategy is able to maintain the dc-link voltage which is not been controlled by the energy conversion. The PI voltage control has been used for the bidirectional converter, in order to control the dc-link voltage. This dc-link voltage is necessary to be maintained in order to transfer the power from the LG generator to the load and to the storage element. By having a low constant dclink voltage, a boost converter can be used in order to increase the output voltage to a reasonable voltage for the energy transfer section. By adding energy storage with the energy conversion, it will not have any effect to the LG outputs that have been controlled before. It indicates that, this part works independently but is still capable to store and to release the energy regarding the power required by the load. The important contribution from this part is that the generated power at the LG remains the same, but how much power that can be transferred to the load is totally depended to the energy flow from the energy storage element, which make the system is robust to load changing. From the results, its show that by increasing the resistive load value, the required power is low so the time taken to store and to discharge the energy is longer than the generated power from the the LG. This means that, the energy storage has a flexible released current time in order to supply the power for the transfer process. 


\section{Chapter 6}

\section{Energy transfer: VOC PI - $H^{\infty}$ power flow control}

This chapter explains the control techniques that have been applied to the 3-phase inverter for a grid connected system. This part is known as the energy transfer where the main focus is to transfer the power that has been captured from the energy conversion and energy storage to the electrical grid. The $H^{\infty}$ current control strategy with the VOC power control have been used together, in order to respond to the power required by the grid. The VOC control consists of a real and reactive power control with feedforward dc-voltage control. A simple synchronisation method has been used in order to allow the power flow from the inverter to the grid can be achieved. Finally, the simulation results have shown the proposed control strategies are able to control the power flow based to the reference power.

\subsection{Description of the inverter at the grid connected circuit}

The inverter is connected to the grid with the filter between them. The function of the filter is to filter the outputs signal that comes out from the inverter. This signal consists of a very high harmonics. The LCL filter has been used in the ERLA system as shown in Figure 6.1 and it is represented by a single phase equivalent circuit. In this circuit, the inverter is connected to the dc-link $\left(V_{d c}\right)$, where this voltage is coming from the boost converter output at the energy storage part. The $u$ is the control signal that generates the PWM signal for the inverter. The MOSFET devices have been used as the power electronics element in the inverter circuit. On the right of Figure 6.1, the filter is connected to the grid source $v_{g}$. For this circuit topology, the power flow is from the inverter to the grid and not from the grid to the inverter. To disconnect the power flow, the circuit breaker $(\mathrm{CB})$ is used in order to separate between the inverter 
to the grid when the inverter output power is zero compared to grid power. The CB is controlled by the $V_{d c}$ logic that determined the on and off of the CB.

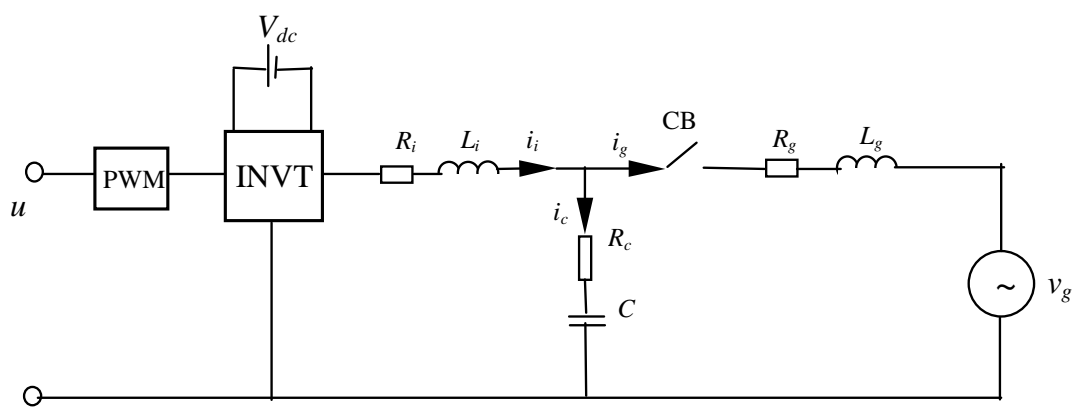

Figure 6.1: Equivalent single phase inverter

The LCL filter value is based on the equations given in [91, 90, 138]. The LCL value is calculated based on the grid and inverter parameters shown in Table 6.1. The inductor that has been designed must have the capability of having $10 \%$ ripple from the rated current. Equation 6.1 is used to calculated the inverter inductance $\left(L_{i}\right)$ :

$$
L_{i}=\frac{\frac{V_{d c}}{2}-V_{p h}}{2 \times \Delta I_{L \max }} \times \frac{D}{f_{s w}}
$$

where $f_{s w}$ is the switching frequency of the inverter, $\Delta I_{\operatorname{Lmax}}$ is $10 \%$ of the rated current of the inverter and $D$ is the duty cycle of the inverter. By substituting the values from Table 6.1 into equation 6.1 the $L_{i}$ can be found as $4 \mathrm{mH}$.

Table 6.1: Design parameters

\begin{tabular}{|c|c|}
\hline Ratings & Value \\
\hline \hline output power for the inverter & $1000 \mathrm{~W}$ \\
\hline dc-link voltage & $300 \mathrm{~V}$ \\
\hline switching frequency $\left(f_{s w}\right)$ & $10 \mathrm{kHz}$ \\
\hline phase voltage $\left(V_{a}\right)$ & $98 \mathrm{~V}$ \\
\hline rated current & $4.7 \mathrm{~A}$ \\
\hline
\end{tabular}

For the capacitor design, it is can be calculated from equation below, with some design procedures given in [91]. The equation is:

$$
C_{f}=0.05 C_{b},
$$

where $C_{b}$ is the base value for the system. The base value can be calculated based on the equation below:

$$
C_{b}=\frac{1}{\omega_{n *} Z_{b}},
$$


where $\omega_{n}$ is the grid frequency in $\mathrm{rad} / \mathrm{sec}$ with $Z_{b}$ is the base impedance given by $28.9 \Omega$. By using equation 6.3 the capacitance base value is $110.14 \mathrm{uF}$ and the capacitor $C_{f}$ can be found to be $5.507 \mathrm{uF}$. After the $L_{i}$ and the $C_{f}$ have been calculated, the next step is to calculated the inductance at the grid connection $\left(L_{g}\right)$. Normally $L_{g}$ is the summation of the transformer leakage reactance and the grid inductance $\left(L_{g}=L_{t f}+L_{g f}\right)$ side. In this simulation the transformer has not been used. Generally, the transformer leakage reactance is the dominant value for the $L_{g}$. In this case, a small value of grid inductance has been selected to represent the $L_{g f}$. The $L_{g f}$ is selected as $15 u H$. In this LCL filter design, the damping resistor $R_{c}$ is used to avoid the resonance at the capacitor. Equation 6.4 shows the equation to calculate the damping resistor :

$$
R_{c}=\frac{1}{3 \times \omega_{\text {res }} \times C}
$$

where the $\omega_{\text {res }}$ is the resonant frequency that is given by $7552 \mathrm{rad} / \mathrm{sec}$. The value for the damping resistor is $10 \Omega$. Table 6.2 shows the values of the LCL filter for inverter grid connection.

Table 6.2: LCL filter values

\begin{tabular}{|c|c|c|c|}
\hline Parameters & value & Parameters & value \\
\hline \hline$R_{i}$ & $0.04 \Omega$ & $L_{i}$ & $4 \mathrm{mH}$ \\
\hline$R_{g}$ & $0.1 \Omega$ & $L_{g}$ & $15 \mathrm{uH}$ \\
\hline$R_{c}$ & $10 \Omega$ & $C$ & $5.5 \mathrm{uF}$ \\
\hline
\end{tabular}

\subsection{Description of controller model for the inverter}

The proposed control strategy is shown in Figure 6.2. This strategy, is a combination of the current control as the inner control loop with the VOC control for the outer control loop. Both controllers must operate together in order to respond to the setting amount of power at the inputs given by $P^{*}$ and $Q^{*}$. It is used to generate the reference currents which are $\left(i_{a}^{*}, i_{b}^{*}, i_{c}^{*}\right)$ for the current control. The $V_{d c}^{*}$ is used to maintain the dc-link voltage at the input inverter, and the $\theta$ is the phase information from the PLL block in order to synchronise the inverter with the grid voltage. The output from these controllers is used to generate the voltage signals that will be added with the grid voltage after the phase-lead low pass filter response. The phase-lead low pass filter is used to compensate the phase delay caused by the filter in order to generate the voltage signal to the PWM signals when it is been synchronised with the grid voltage. This phase-lead filter is built to have a slightly higher gain than 1 , and the phase angle have 
a small lead of phase angle from the fundamental frequency. In this work the value for this phase-lead low pass filter is given by [89]:

$$
T(s)=\frac{825(s+20)}{(s+300)(s+500)} \text {. }
$$

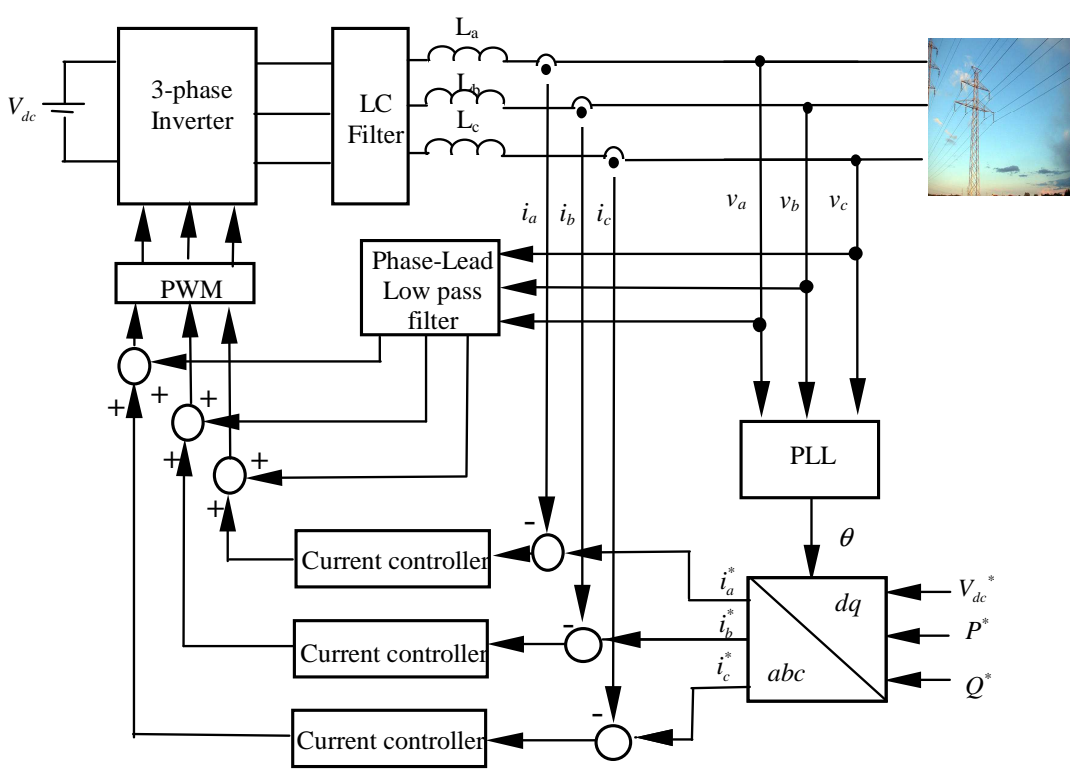

Figure 6.2: The control strategy for inverter-grid connection in ERLA energy transfer application

\subsubsection{VOC-PI for outer power loop}

The outer controller for the inverter consists of reactive power, real power and the dcvoltage control shows in Figure 6.3. The $V_{d c}^{*}, P^{*}$ and the $Q^{*}$ are the references target for the VOC-PI controller. The $V_{d c}$, is used to control the dc voltage input at the inverter. This is to make sure the dc inverter input voltage can be maintained when the energy is been transferred from the inverter to the grid. This dc voltage controller will be feedforwarded to the real power controller. Three PI controllers have been applied to the VOC as shown in Figure 6.3. The outputs from real, reactive and dc voltage controller will generate the $I_{d}^{*}$ and $I_{q}^{*}$ that respond to the real and reactive targets value. The reason why it is called the VOC is because this generated references current behave like the voltage oriented output to the normal real and reactive power calculation given in [99]. The value of $I_{d}^{*}$ and $I_{q}^{*}$ can be changed depending on the setting value of $P^{*}$ and $Q^{*}$ for different power required. The $d q-a b c$ transformation is used to generate the 3 -phase references current for the inner loop control $\left(i_{a}^{*}, i_{b}^{*}\right.$ and $\left.i_{c}^{*}\right)$ after the power 
control application. To generate the 3-phase references current that are synchronise with the grid voltage, the PLL is used in order to give the phase information of the grid voltage. Finally, the 3-phase current can be generated with the same magnitude but different phase angle for each phase that is used in natural frame configuration for the current control.

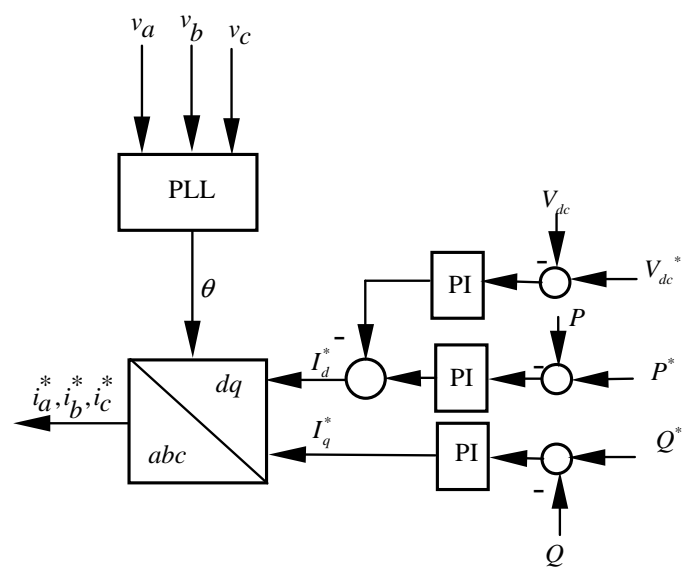

Figure 6.3: The block diagram of power control with VOC control strategy

\subsection{2 $H^{\infty}$ current control design for inner control loop}

Figure 6.4 shows the control block inside the current control which is used the $H^{\infty}$ control theory method. The function of the $H^{\infty}$ control is to send a signal to the PWM which gives minimum tracking error value in order to inject the current to the grid. The $G$ and $K$ in Figure 6.4 represent the plant and the stabilising compensator that have been generated from the $H^{\infty}$ control theory. The advantage of this control theory is that the disturbances $w$ which are the grid voltage and the target references current can be included in the compensator design calculation. From Figure 6.4, the output generated from the $K$ is $u$ where it used to generate the PWM signals, meanwhile the $y$ is the target output that coming from the error between the current reference and the output current at the inverter. It can be written as:

$$
y=e=i_{\text {ref }}-\left[i_{a} / i_{b} / i_{c}\right]
$$

\subsubsection{State-space model of the inverter for $H^{\infty}$ control calculation}

To derive the state-space equation from the inverter plant, it is done by simplifying Figure 6.1 to Figure 6.5. It shows that the state-space equation can be formulated 


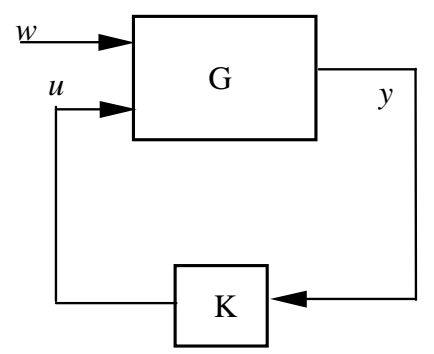

Figure 6.4: The $H^{\infty}$ current control for the inner loop controller

based only on the LCL configuration, where $u$ is the average voltage for the output voltage of the inverter $[100,101]$. The average technique is applied to the inverter voltage, where makes the on and off function of the switches for the power electronics devices can be ignored.

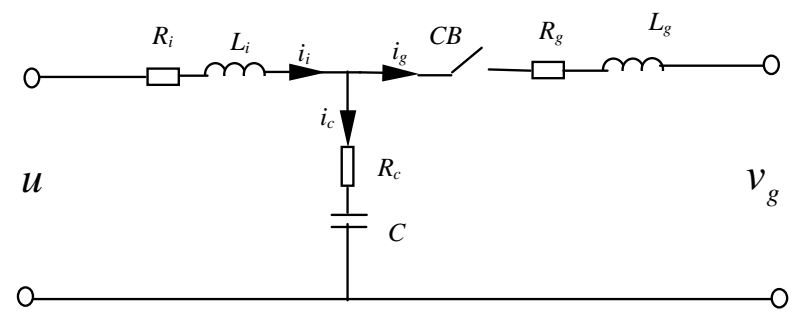

Figure 6.5: Single phase representation of the inverter

Before the state-space equations can be formulated, the state variables of the inverter plant must be determined. From Figure 6.5 the state variables are given by $x=\left[\begin{array}{lll}i_{i} & i_{g} & v_{c}\end{array}\right]^{T}$. Two common laws used in circuit analysis which are the Kirchhoff's voltage law and the Kirchhoff's current law have been applied to Figure 6.5. The equations can be written when the $\mathrm{CB}$ is closed and it given by:

$$
\begin{aligned}
\frac{\delta i_{i}}{\delta t} & =-i_{i}\left(\frac{R_{i}+R_{c}}{L_{i}}\right)+i_{g} \frac{R_{c}}{L_{i}}-\frac{v_{c}}{L_{i}}+\frac{u}{L_{i}} \\
\frac{\delta i_{g}}{\delta t} & =-i_{g}\left(\frac{R_{g}+R_{c}}{L_{g}}\right)+i_{i} \frac{R_{c}}{L_{g}}+\frac{v_{c}}{L_{g}}-\frac{v_{g}}{L_{g}} \\
\frac{\delta v_{c}}{\delta t} & =\frac{i_{i}-i_{g}}{C} .
\end{aligned}
$$

From Figure 6.4, the input for the plant $G$ consists of two inputs. The first input is disturbance $w$ where from equation 6.7 is the $v_{g}$. This disturbance also includes the reference current generated from the outer power loop control. The matrix disturbance can be written in state-space as $w=\left[\begin{array}{ll}v_{g} & i_{\text {ref }}\end{array}\right]^{T}$. The second input to the $G$ is 
$u=[u]$. This parameter can be found in equation 6.6. By rearranging the state-space matrix which includes the state variables, the state disturbance and the state input, the state-space equation of the plant $G$ can be written as:

$$
\dot{x}=A x+B_{1} w+B_{2} u .
$$

As shown in equation 6.5, the output is the error between the output value and the reference value of the inverter which can be summarised as:

$$
e=C x+D_{1} w+D_{2} u
$$

where

$$
\begin{aligned}
A & =\left[\begin{array}{ccc}
-\left(\frac{R_{i}+R_{c}}{L_{i}}\right) & \frac{R_{c}}{L_{i}} & \frac{-1}{L_{i}} \\
\frac{R_{c}}{L_{g}} & -\left(\frac{R_{g}+R_{c}}{L_{g}}\right) & \frac{1}{L_{g}} \\
\frac{1}{C} & -\frac{1}{C} & 0
\end{array}\right] \\
B_{1} & =\left[\begin{array}{cc}
0 & 0 \\
\frac{-1}{L_{g}} & 0 \\
0 & 0
\end{array}\right] \\
B_{2} & =\left[\begin{array}{c}
\frac{1}{L_{i}} \\
0 \\
0
\end{array}\right] \\
C & =\left[\begin{array}{lll}
0 & -1 & 0
\end{array}\right] \\
D_{1} & =\left[\begin{array}{ll}
0 & 1
\end{array}\right] \\
D_{2} & =\left[\begin{array}{ll}
0 &
\end{array}\right.
\end{aligned}
$$

By arranging equations 6.9 and 6.10 into matrix notation, the inverter plant $G$ can be written as:

$$
G=\left[\begin{array}{c|cc}
A & B_{1} & B_{2} \\
\hline C & D_{1} & D_{2}
\end{array}\right] .
$$

\subsubsection{2 $H^{\infty}$ formulation for current controller design}

Figure 6.6 shows the arrangement of the $H^{\infty}$ general control block diagram in order to calculate the $K$. It consists of $W_{1}$ and $W_{2}$ that are known as the weighting functions. The function for $W_{1}$ is used to make the output $e$ to improve the tracking error performance and $W_{2}$ as a function to calculate the full rank value needs for the $H^{\infty}$ control theory [100, 101, 114]. To have good error tracking, the low pass filter was used in $W_{1}$ and first order transfer function for $W_{2}$ was selected. The state-space matrix notation for the $W_{1}$ and $W_{2}$ are given by: 


$$
W_{1}=\left[\begin{array}{c|c}
A_{W_{1}} & B_{W_{1}} \\
\hline C_{W_{1}} & D_{W 1}
\end{array}\right], W_{2}=\left[\begin{array}{l|l}
A_{W_{2}} & B_{W_{2}} \\
\hline C_{W_{2}} & D_{W_{2}}
\end{array}\right] .
$$

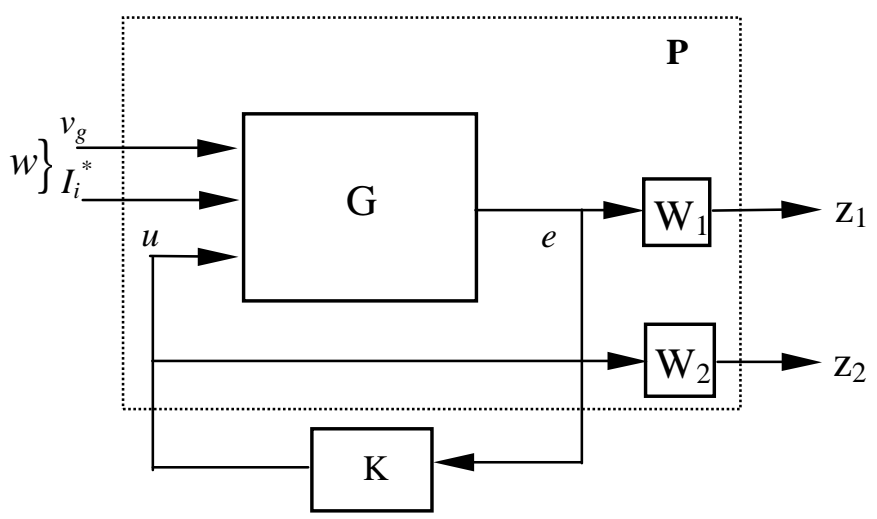

Figure 6.6: The block diagram to formulate the $H^{\infty}$ control theory for current controller

The dotted line shows in Figure 6.6 is the new extended plant $P$ that consisting of the $G, W_{1}$ and $W_{2}$ and the inputs. This will create the new regulated performance outputs which are given by $z_{1}$ and $z_{2}$. The $z_{1}$ and $z_{2}$ can be formulated as

$$
\begin{aligned}
z_{1} & =e \times W_{1} \\
& =\left[\begin{array}{c|cc}
A & B_{1} & B_{2} \\
\hline C & D_{1} & D_{2}
\end{array}\right]\left[\begin{array}{c|c}
A_{W_{1}} & B_{W_{1}} \\
\hline C_{W_{1}} & D_{W_{1}}
\end{array}\right]\left[\begin{array}{l}
w \\
u
\end{array}\right] \\
& =\left[\begin{array}{cc|cc}
A & 0 & B_{1} & B_{2} \\
B_{W_{1}} C & A_{W_{1}} & B_{W_{1}} D_{1} & B_{W 1} D_{2} \\
\hline D_{w_{1}} C & C_{w_{1}} & D_{w_{1}} D_{1} & D_{w_{1}} D_{1}
\end{array}\right]\left[\begin{array}{l}
w \\
u
\end{array}\right],
\end{aligned}
$$

and for

$$
\begin{aligned}
z_{2} & =W_{2} \times u \\
& =\left[\begin{array}{l|ll}
A_{W_{2}} & 0 & B_{W_{2}} \\
\hline C_{W_{2}} & 0 & D_{W_{2}}
\end{array}\right]\left[\begin{array}{l}
w \\
u
\end{array}\right] .
\end{aligned}
$$

By combining equations 6.12 and 6.13 , the extended plant $P$ can be obtained as:

$$
P=\left[\begin{array}{ccc|cc}
A & 0 & 0 & B_{1} & B_{2} \\
B_{w_{1}} C & A_{w_{1}} & 0 & B_{w_{1}} D_{1} & B_{w 1} D_{2} \\
0 & 0 & A_{W_{2}} & 0 & B_{W_{2}} \\
\hline D_{w_{1}} C & C_{w_{1}} & 0 & D_{w_{1}} D_{1} & D_{w_{1}} D_{1} \\
0 & 0 & C_{W_{2}} & 0 & D_{W_{2}} \\
\hline C & 0 & 0 & D_{1} & D_{2}
\end{array}\right] .
$$




\subsubsection{3 $H^{\infty}$ controller design}

Table 6.2 shows the parameters that are used to calculated the compensator controller value. As the target output is the tracking error, the $W_{1}$ transfer function is chosen for low pass filter at $f=50 \mathrm{~Hz}$. The values for the weighting functions are given by:

$$
W_{1}=\frac{0.5(s+492980)}{s+314}, W_{2}=\frac{0.25 s+1245}{s+5000} .
$$

By using the hinfsyn command that is available in MATLAB software, the compensator value for the $K$ can be calculated as shown in equation 6.16. From equation 6.16 , it is shown that the zeros and poles are located on the left hand side of the root locus graph. This shows that the generated controller value is stable and it is suitable to be used as a compensator $K$. The final equation value is given by:

$$
H^{\infty}=\frac{381341062651608.1(s+4.603 e 006)(s+6542)(s+255.1)}{(s+3.001 e 012)(s+4.656 e 006)(s+6662)(s+313.4)} .
$$

In this equation, some of the poles and zeros location are located near to each other. These values can be cancelled to each other in order to make the control transfer function more stable and easy to implement. From equation 6.16 one of the denominator which is the $(s+3.001 e 012)$, can be approximated to cancel the gain value at 381341062651608.1 without causing any changes in the performance that has been justified in $[21,89]$. The reduced transfer function for the controller is given by:

$$
K=H^{\infty}=\frac{127(s+255.1)}{(s+313.4)} .
$$

\subsubsection{Stability evaluation for the $H^{\infty}$ control}

In order to guarantee the stability of the system, the value of the $K$ must be included in the stability calculation. The stability calculation is based on Figure 6.7.

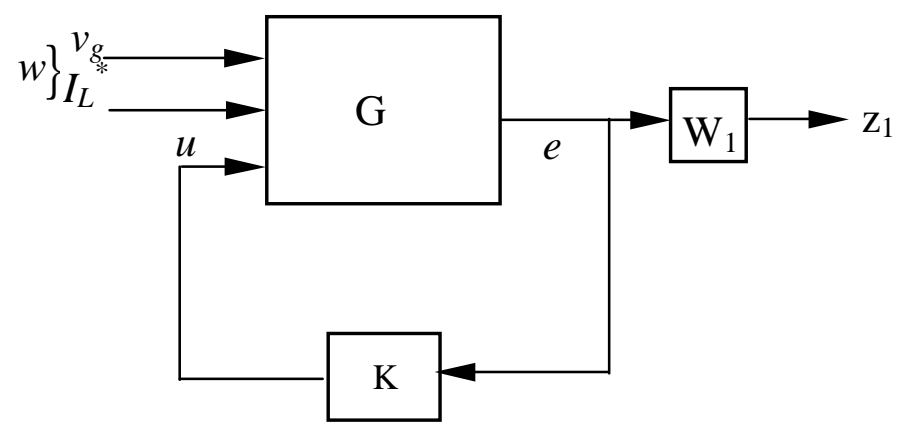

Figure 6.7: The block diagram of stability structure for current control 
The stability calculation is based on the closed loop system from the $z_{1}$, which is the new regulated output vector to the output $e$. This condition is based on the transfer function where it can be written as $T_{z_{1} W_{1}}$. The condition needs to be satisfied according to the stability condition is given as:

$$
\left\|T_{z_{1} W_{1}}\right\|<1 .
$$

The stability value is calculated based on the state-space technique. The state-space equation from Figure 6.7 is included the compensator $K$ and by assuming the $w=0$, it can be written as:

$$
\begin{aligned}
& \dot{x}=A x+B_{2} u \\
& e=C x+D_{2} u,
\end{aligned}
$$

where the value of $A, B_{2}, C$ and $D_{2}$ are the same in equation 6.11. The state-space equation for the $K$ can be given as $K=\left[\begin{array}{c|c}A_{K} & B_{K} \\ \hline C_{K} & D_{K}\end{array}\right]$ and it can be written in state space format by:

$$
\begin{aligned}
u & =C_{K} x_{c} \\
\dot{x_{c}} & =A_{K} x_{c}+B_{K} e .
\end{aligned}
$$

By substituting equation 6.20 into equation 6.18 , the state variable of the plant can be written as:

$$
\dot{x}=A x+B_{2} C_{K} x_{c} .
$$

For the state variable of the $K$ it can be found by substituting equations 6.19 and 6.20 into equation 6.21 where the final equation is given by:

$$
\begin{aligned}
& \dot{x_{c}}=A_{K} x_{c}+B_{K} C x+B_{K} D_{2} C_{K} x_{c} \\
& \dot{x_{c}}=\left(A_{K}+B_{K} D_{2} C_{K}\right) x_{c}+B_{K} C x .
\end{aligned}
$$

For the $e$, the state equation can be written by substituting equation 6.20 into equation 6.19 and it is shown as:

$$
e=C x+D_{2} C_{K} x_{c}
$$

By arranging equations $6.22,6.23$ and 6.24 , the state-space matrix equation of the 
feedback loop from $G$ to $K$ can be written as:

$$
E=\left[\begin{array}{cc|c}
\left(A_{K}+B_{K} D_{2} C_{K}\right) & B_{K} C & 0 \\
B_{2} C_{K} & A & 0 \\
\hline D_{2} C_{K} & C & 0
\end{array}\right]
$$

By multiplying equation 6.25 with $W_{1}$, the following equation gives the new matrix in order to calculate the stability value as:

$$
\begin{aligned}
T_{z_{1} W_{1}} & =E \times\left[\begin{array}{c|c}
A_{W_{1}} & B_{W_{1}} \\
\hline C_{W_{1}} & D_{W_{1}}
\end{array}\right] \\
& =\left[\begin{array}{ccc|c}
\left(A_{K}+B_{K} D_{2} C_{K}\right) & B_{K} C & 0 & 0 \\
B_{2} C_{K} & A & 0 & 0 \\
0 & 0 & A_{W_{1}} & B_{W 1} \\
\hline D_{2} C_{K} & 0 & C_{W_{1}} & D_{W_{1}}
\end{array}\right] .
\end{aligned}
$$

Equation 6.26 can be developed in the MATLAB environment and uses the normhinf, to find the stability value. The stability from equation 6.26 is given by $\left\|T_{z_{1} w_{1}}\right\|=0.5008$ and this value is stable which is stated in [89].

\subsection{Simulation results}

The proposed control strategies for power control and current control were used in order to understand the complete process for the ERLA system. By combining this part with the other parts in the ERLA system and running the simulation for the power flows strategy, the complete process from beginning to end can be achieved. The parameter values are based on Tables 6.1 and 6.2 for the energy transfer process were included in the configuration parameters.

In this simulation the inverter was considered to be connected to the grid directly without the step up transformer and it uses the PLL for the synchronisation process of the inverter with the grid voltage. Two simulation conditions were conducted to see the changing of the transferred power to the grid. The simulations were carried out using the MATLAB Simulink package with maximum step size of 0.01 miliseconds.

For the first simulation, the target value for real and reactive power were set at $P^{*}=1000 \mathrm{~W}$ and $Q^{*}=0 \mathrm{VAR}$. This is to ensure that only the real power can flow to the load while maintaining the power factor at unity. In order to see the response of the power control with different reference power, the second simulation was conducted by changing the reference power $P^{*}=500 \mathrm{~W}$ to $P^{*}=1000 \mathrm{~W}$ after $t=3 \mathrm{~s}$. At the same time, the reactive power was maintained at $Q^{*}=0 \mathrm{VAR}$. The simulations were run from $t=0 \mathrm{~s}$ to $t=7 \mathrm{~s}$. 


\subsubsection{With the reference power of $P^{*}=1000 \mathrm{~W}$ and reactive of $Q^{*}=$ OVAR}

For the first simulation, the system outputs response to the real and reactive power are shown in Figures 6.8, 6.9 and 6.10. Figure 6.8 are the outputs at the energy conversion part, where the generated power and the inductor current remain at the same magnitude and same profile as in Chapter 4 where it have been explained before. Of importance here is that, when the transfer power happens, the deceleration profile is still maintained constant as shown in Figure 6.8c. This indicates that, the CBB converter with $H^{\infty}$ current control is working properly during the simulation without any effect due to the power transfer.

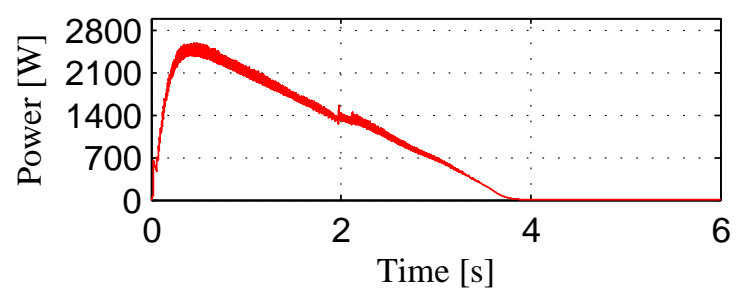

(a) power generated during landing

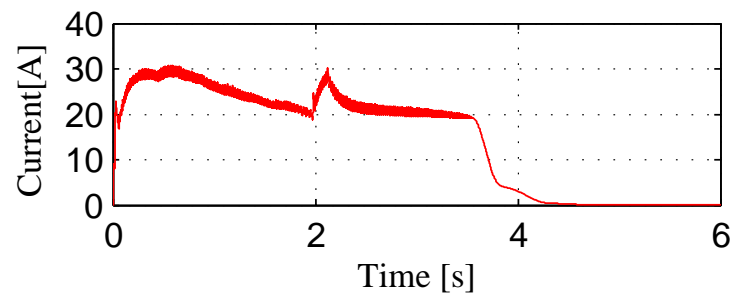

(b) inductor current at CBB converter

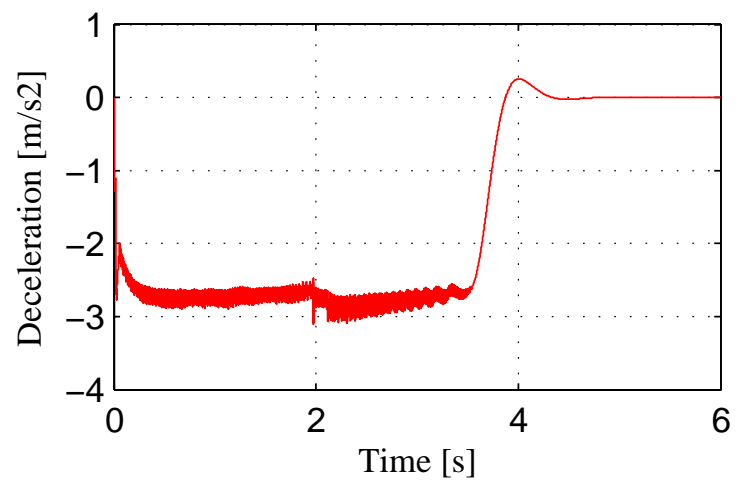

(c) deceleration speed during landing

Figure 6.8: Simulation outputs at energy conversion part when all the parts combined together

For the energy storage section the outputs are shown in Figure 6.9. From these outputs the dc-link voltage between the energy conversion section and the energy storage section are maintained at the target reference voltage shows in Figure 6.9a while the 
boost converter is controlled to the target shows in Figure $6.9 \mathrm{~b}$. It explains that, the energy storage is able to store the excessive energy at the beginning of the landing and release the energy when the energy from the landing is lower than the energy need to be transferred to the grid.

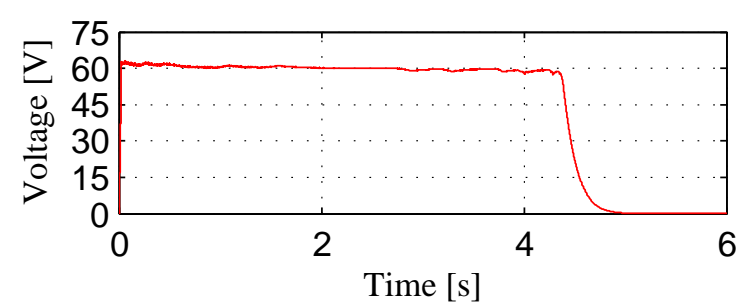

(a) dc-link voltage between the energy conversion and energy storage

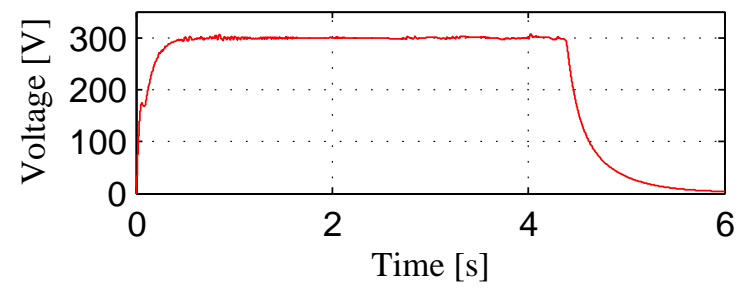

(b) output voltage at the boost converter

Figure 6.9: Simulation outputs at energy storage part when all the parts combined together

For the energy transfer section, the outputs are shown in Figure 6.10. Figures 6.10a and $6.10 \mathrm{~b}$ show the real power and reactive power that has been controlled by using the VOC- $H^{\infty}$ controllers at the energy transfer part. It shows that, the real power is maintained at reference power $P^{*}$ during the simulation time and the small amount of reactive power is been transferred to the grid. This means that, the power can be transferred referring to $P^{*}=1000 \mathrm{~W}$. The time taken to supply this power is more longer then the time at the power generated shows in Figure 6.8a. This is because, the calculated average power for $4 \mathrm{~s}$ is $1250 \mathrm{~W}$ but the reference power is set at $1000 \mathrm{~W}$ which is below the calculated average power. Due to this, the time taken to store and time to release the energy from the ultracapacitor is longer then the time of the generated power shows in Figure 6.8a. It also explains that, the energy power transfer has the same situation when the load is higher which has been shown in Figure 5.9 in energy storage part. Figure 6.10c shows the line to line output voltage at the inverter which is the same as the line to line grid voltage. 


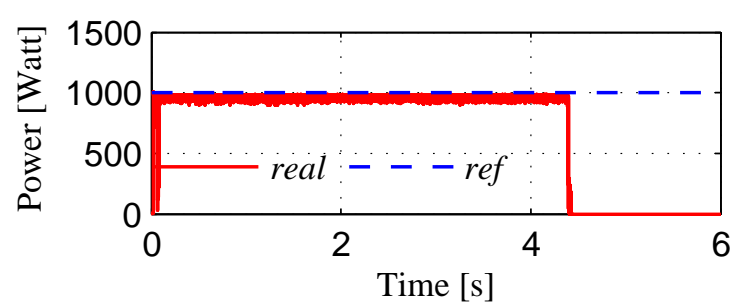

(a) real power transfer with measured value (real) and target value (ref)

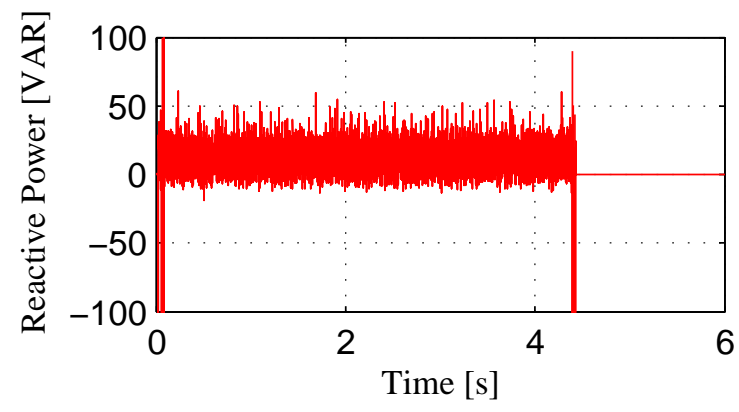

(b) reactive power transfer to the grid

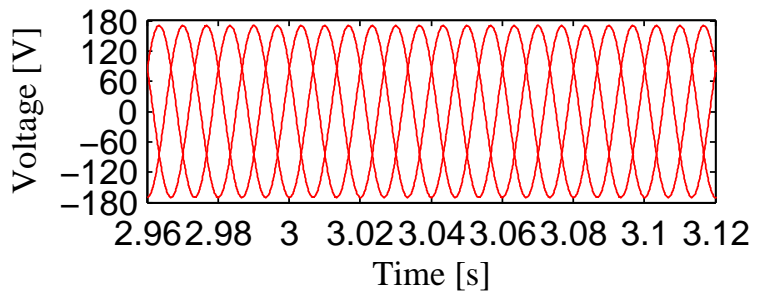

(c) output voltage at $v_{g}$

Figure 6.10: Simulation outputs at energy transfer when $\mathrm{P}$ is set to $P^{*}=1000 \mathrm{~W}$

Figure 6.11 shows the response of the $H^{\infty}$ current controllers at the CBB converter and the inverter. Both of the $H^{\infty}$ current controllers that have been designed are able to track the reference currents given for each controller. For Figure 6.11a the input current of the CBB converter is able to track the reference current which is set at $20 \mathrm{~A}$ for the current control at the CBB converter. In the mean time, Figure $6.11 \mathrm{~b}$ shows the inverter output current is tracked the reference generated current from the VOC-PI controller which is determined by the amount of power transfer to the grid. Here it indicates that, these controllers can be used to transfer the energy between the energy source and the grid without any problems. 


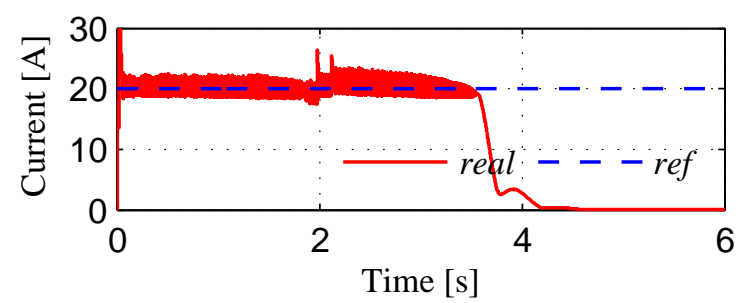

(a) $H^{\infty}$ current control at CBB converter

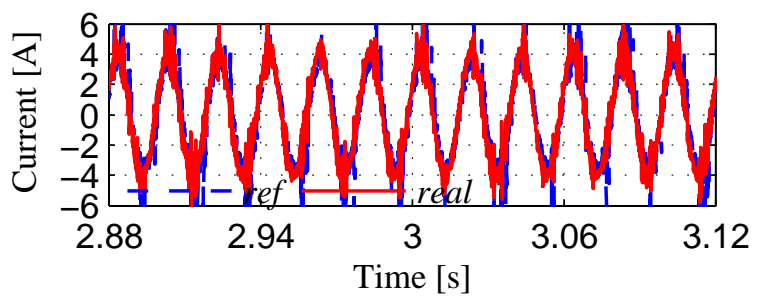

(b) $H^{\infty}$ current control at inverter

Figure 6.11: $H^{\infty}$ current controllers response in the ERLA system

Figure 6.12 shows the synchronisation mechanism between the voltage inverter and the voltage grid. It happens when the grid voltage are being feed-forward shown in Figure 6.2 though the phase-lead low pass filter and add with the signal generated from the $H^{\infty}$ current control. Meanwhile, Figure $6.12 \mathrm{~b}$ shows the voltage drop at the grid inductor during the synchronisation which is less then $1 \mathrm{~V}$. This indicates both system is synchronised.

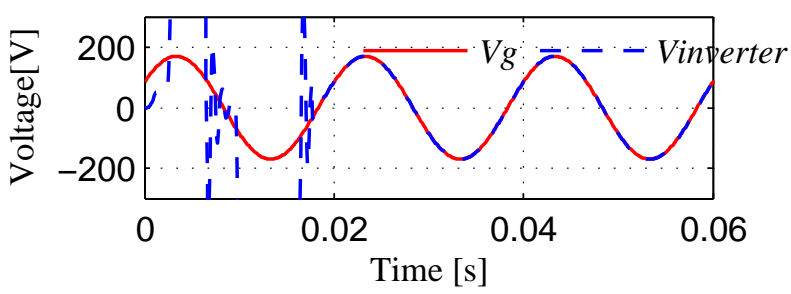

(a) voltage synchronisation

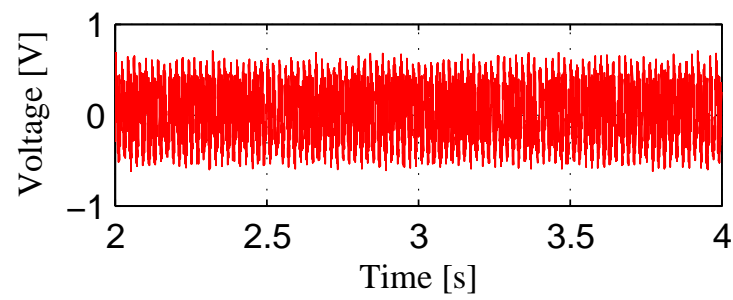

(b) voltage drop on grid interface inductor $v_{g}-v_{i}$

Figure 6.12: Synchronisation mechanism between grid voltage $v_{g}$ and inverter voltage $v_{i}$ 


\subsubsection{With $P^{*}=500 \mathrm{~W}$ from $t=0$ s to $t=3$ s and with real $P^{*}=1000 \mathrm{~W}$ after $t=3$ s and $Q^{*}=0$ VAR}

The simulation was repeated with the given values for the $P^{*}$ and $Q^{*}$ change at the different times in order to see the response of the controller and also to see the time taken to release the energy from the energy storage and when the energy source at energy conversion is zero. Figure 6.13 shows the response from the energy conversion part which has the same profile and magnitude as in Figure 6.8. This indicates that no change has happen to the energy conversion section.

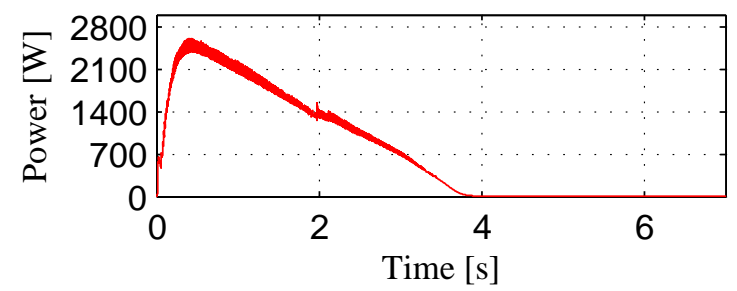

(a) power generated during landing

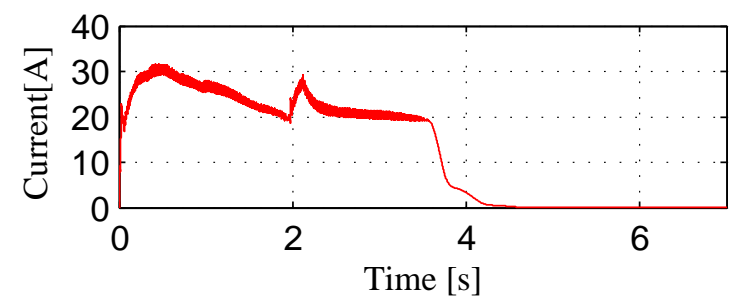

(b) inductor current at $\mathrm{CBB}$ converter

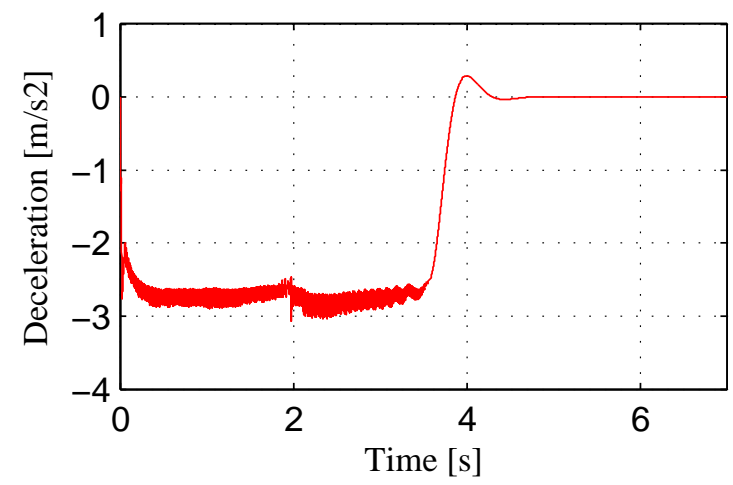

(c) deceleration speed during landing

Figure 6.13: Simulation outputs at energy conversion part when all the parts combined together

From Figure 6.14, the dc-link voltage and the boost output voltage are able to be maintained at the target value. At the same time, the time for voltages to go zero is 
longer than the time shown in Figure 6.9. This is because the power that has been transferred to the grid is $500 \mathrm{~W}$ which is half of the average power at $1250 \mathrm{~W}$ from $0 \mathrm{~s}$ to $3 \mathrm{~s}$. It shows that the energy storage part is capable of charging and discharging the energy with longer time regarding the power needed by the grid side.

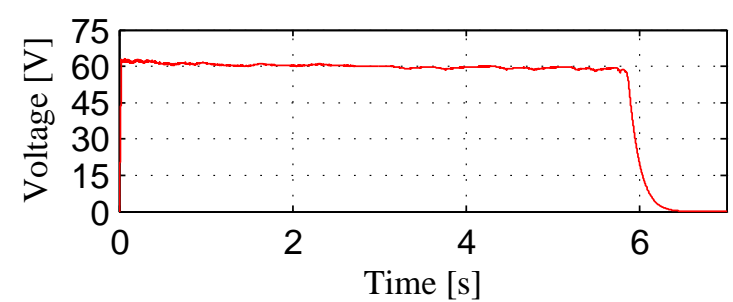

(a) dc-link voltage between the energy conversion and energy storage

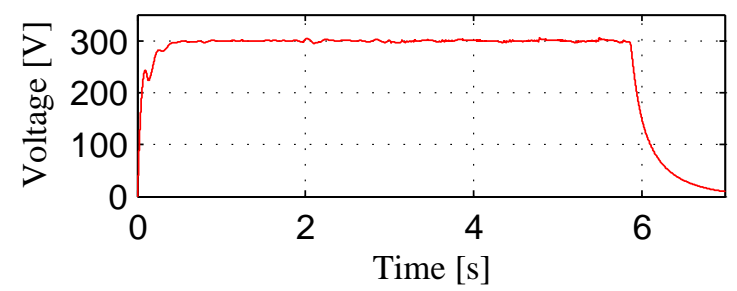

(b) output voltage at the boost converter

Figure 6.14: Simulation outputs at energy storage part when all the parts combined together

Figure 6.15 shows the results at the energy transfer section. Figures $6.15 \mathrm{a}$ and $6.15 \mathrm{~b}$ show the real and reactive power response to the step change after $t=3 \mathrm{~s}$. It shows that, the controllers force the measured power at the inverter to follow the reference power from $500 \mathrm{~W}$ to $1000 \mathrm{~W}$. Here, the reactive power was maintained at 0VAR. The time taken for Figure 6.15 to finish is the same as the time taken in Figure 6.14 due to continuous power supply from the energy storage when after the generated energy is finished that is shown in Figure 6.13a. 


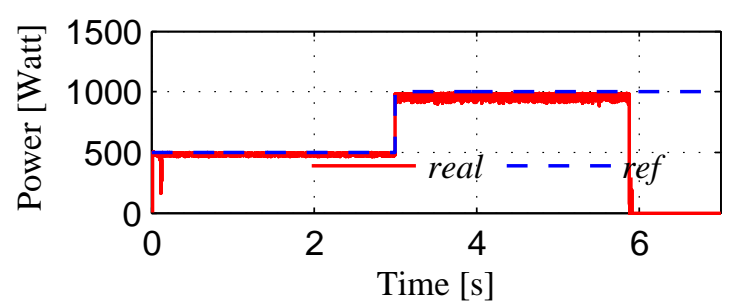

(a) real power transfer with measured value (real) and target value (ref)

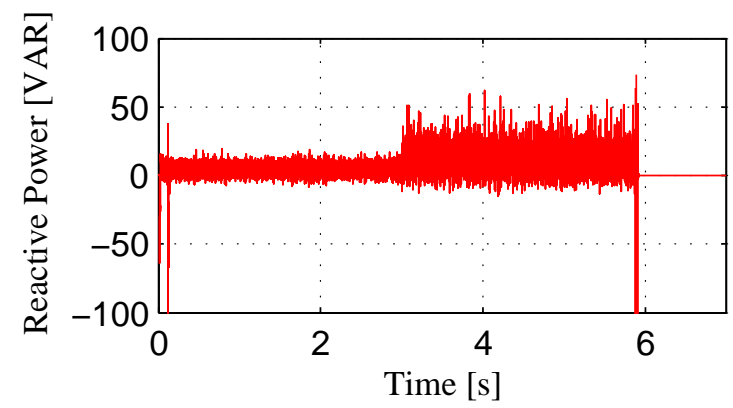

(b) reactive power transfer to the grid

Figure 6.15: Simulation outputs at energy transfer when $\mathrm{P}$ has a step response from $P^{*}=500 W$ to $1000 W$

Figure 6.16 shows the current response at the CBB converter and the inverter which are used the $H^{\infty}$ current control in order to give good tracking performance between the reference value and the control value. Figure 6.16a shows the tracking current at the CBB current controller. It shows that, by using the $H^{\infty}$ current control, the current controller is able to track the reference current set by the CBB converter. For the inverter current tracking, the result is shown in Figure 6.16b. It demonstrates that, when the power is changed at $t=3 \mathrm{~s}$, the generated references current are changed due to the step changed of the power that caused the references current to change. Due to this condition, both controllers are working properly in order to deliver the power to the grid. 


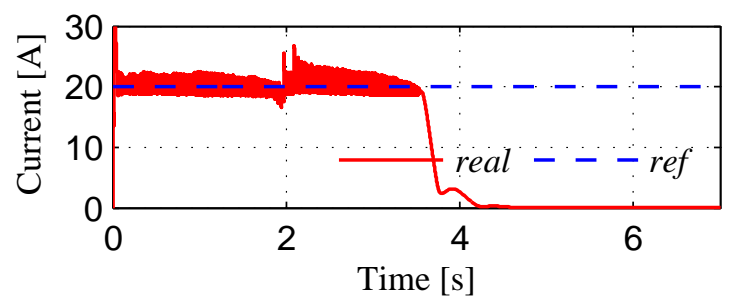

(a) $H^{\infty}$ current control at CBB converter

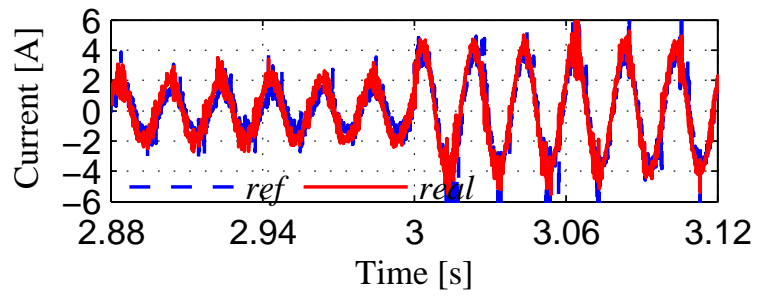

(b) $H^{\infty}$ current control at inverter

Figure 6.16: $H^{\infty}$ current controllers response in the ERLA system

\subsection{Concluding remarks}

In conclusion for this chapter, the energy transfer part, which consists of a 3-phase inverter and the LCL filter are used to transfer the energy from the energy conversion and the energy storage to the grid. The VOC-PI control is used for the power control while the $H^{\infty}$ control theory is used for the current control for the inverter. The VOC control is able to respond to the changing power, when there has any changed at the reference power which is given by the load power. This controller adopts concept that has been well established in power control applications in the inverter, but in this work it is used to generate the references current for the current controller. As is known, the current controller strategy is to inject a suitable amount of current according to the reference current with less harmonic effect. This is because the design compensator is included the LCL filter components in order to improve the harmonics current to the grid. From the results, by using these control strategies to the inverter, it is capable of transferring the power from the source to the load by depending to the requested load power. At the same time it also gives a small voltage different between the inverter voltage and the grid voltage, which indicates that a synchronisation has happened for both system. At the end, by combining all the energy parts that represent the ERLA system, the energy generated from the landing aircraft can be transferred to the grid in order to be as a electrical energy source. 


\section{Chapter 7}

\section{Conclusions and Future Work}

\subsection{Conclusions}

Obtaining electric energy from landing aircraft is a new idea. In order to understand the power transformation process, all the parameters that have been used in this work is based on the maximum power that can been collected from the test rig that has been build in the lab. The test rig is consists of the flywheel, the generator machine, and the power processing devices in order to behave as a mimic landing aircraft. This test rig can be expanded to the real application if the maximum power which has been generated from the landing is been calculated. The test rig is necessary in order to understand the behaviour of the process by using the small scale of the system. This is because all the electrical components value that have been used are determined from the available maximum power. This test rig network is shown in the Appendix A. The idea of this work is to show the process from the beginning of the generated power to the power transfer process to the electrical grid in a simulation environment. For this process the ERLA system can be divided into three sections which are energy conversion, energy storage and energy transfer. This initial study has opened up a new understanding about the possibility of using the kinetic energy during landing as a alternative power source for the electricity power supply.

Firstly, energy conversion is used to convert the kinetic energy to electrical energy. The aims for this part are to control the deceleration, and at the same time, control the force that will change the generated output power during landing which is determined the successful of objectives 2 and 3. This can be done by using the LG, the diode rectifier and the CBB converter. Here, the LG is responded to the input speed from the aircraft and produces a variable magnitude current, voltage and frequency with the uncontrolled force as the outputs. To understand the process for the LG, the LG was modelled in two models. First it was modelled in 3-phase model and than in the $d q$ model. Both models show the possibility of the LG where it uses the speed as the input and produces 
the force, voltage and current at the outputs. In these models, no control strategy was put in placed in order to reduce the number of the controllers. Then, the diode rectifier was used to convert the ac input to dc output, which is contains high ripple with decreasing current or voltage magnitude. Because of this, the CBB converter was used in order to control the input current and at the same time to maintain the constant force and the deceleration at the LG side. In others words, by applying the current control to the CBB converter, the LG outputs also can be controlled. This will benefit the passengers comfort due to constant braking force applied that has been generated at the LG output. It is also will change the generated power, from exponential decay power profile to linearly decreasing power where it can be used to determine the average and the maximum power output. The current control with $H^{\infty}$ control theory was designed that gives ability to give small tracking error and also includes the disturbance inputs for the controller calculation. It shows that, by using this control strategy the generated power can be changed from the exponential decay profile to linear profile where it is important in order to calculate the size of the energy storage element and to determine the maximum power that can be transferred to the load.

The next part of the process is the energy storage. The ultracapacitor was used as the energy storage element. This part is necessary, because at the beginning of the landing, the generated power is higher and then it will linearly decrease to zero along the time. This initial power cannot be directly transferred to the load or grid because it will cause damage to the grid. To deal with this, the ultracapacitor with bidirectional converter were used to control the power flow in or out from the ultracapacitor. As a result, the voltage controller with PI control was used in order to maintain the dc-link voltage which was not been controlled during the energy conversion. In the mean time, it also capable to absorb or release the energy from the ultracapacitor. The boost converter was used at the energy storage part in order to increase the input dc-link voltage to the specific output voltage for the inverter application, which in this work the voltage was increased from $60 \mathrm{~V}$ to $300 \mathrm{~V}$. From the simulation results, when this part was combined with the energy conversion, the results responded well to the load changing. When the load is higher, the time taken to release the energy from the energy storage is longer because the power required by the load is lower than the average generated power. If the power required by the load is the same as the average power value, the time to release the energy by the ultracapacitor is the same as the generated power from the LG.

Then, the energy transfer was used to transfer the power from the main source and the ultracapacitor power to the grid. The transferring power was determined by the required power from the load. Here, the VOC-PI power control with $H^{\infty}$ current 
control were used in order to transfer the power, requested by the load. The LCL filter was used to ensure the injected current contains less harmonics that is good for the grid connection system. In the simulation results, where all the parts and the proposed controllers were been put together, two simulations with various settings power have been analysed. The first simulation was conducted when the constant power is required by the load all the times. It shows that, all the controllers can work properly from the LG to the inverter. The generated power from the inverter also response to the required power by the load. The second simulation was conducted when the power changes happen during the simulation time. There was a changed from low power to higher power input power. Here, the results show that, the power can be transferred to the grid within longer time compared to the generated power at the LG. This is because the required load power was lower than the average power generated by the LG that caused the injected current is small than the rated current.

As a conclusion from this work, the kinetic energy produced from the aircraft can be converted to electrical energy using these proposed controllers and converters. This study also shows that the kinetic energy from the landing aircraft can be converted, stored and transferred and become as a electricity generation source.

\subsection{Recommendations for future work}

In this research, the verification and the understanding of the processes starting from the landing to the power transferred to the grid have been conducted in the simulation environment. Based on the studies and works that have been carried out, it is possible to harvest the energy from the landing aircraft but some findings and future works need to be considered. The finding that has been recognised here, is especially regarding to the output power. Here, if the requested power is higher than the generated power the ultracapacitor is not able to absorb the power due to the low rated voltage. To solve this condition the ultracapacitor can be replaced by the battery. This is because the current inside the battery can be controlled to the rated value. Other findings is regarding to the length of the linear generator that can be used if the real landing is applied. By increasing the length of the LG is will increase the losses from the LG where it will affect the electricity generation outputs from this alternative energy.

Although these findings limit the advantages in real system but in terms for military application it gives some advantages. For example, this work can be implemented at the aircraft carrier which is used short platform for landing and takeoff. In this strategy no energy storage will be used but the generated power is directly supplied for takeoff process. For this technique, the aircraft fighter can carry more weapon and used less fuel. For the industry purposes, this application can be applied during the movement 
of the elevator. It is where the generated power during up and down movement of the elevator can be transformed back to elevator motor operation which can reduce the energy building bills.

From these findings, some future works can also be done in order to improve the system. It can be separated into two major elements, which are the mechanical element and the electrical element. The mechanical element basically focuses on the LG itself. In the ERLA application, the LG is been placed underneath the runway. This will increase the cost if it want to be implemented in practical. The other ways is placed the LG along the side of the runway. But for this concepts the aircraft tyres need to be changed to use more heavy metal material because to became as a rotor likes the normal LG operation. The study to the mechanical force impact on the runway is also needed to be considered where it will break the LG if the LG is not been build to absorb this high impact landing. The gap between the runway and the LG also need to be considered where the energy is easily to be dissipated between this gap to the earth and will increase the power losses or less generated power to the grid. Another factor is the human factor which it is the most important factor in aircraft response. It is believed that the force impact factor is contributed to the comfort of the passengers. This force is determined by the braking force as explained in [139].

For the electrical part some improvement especially at the power conditioning parts where the power management/power monitoring system can be included. By including this system, the ERLA system can be controlled by the operators. This is when, the operators can see the amount of generated power and the amount of power needs to be transferred from the system to the grid. In this case, if both power is not matched, the operators can match the power. As known high transient power will be generated at the beginning of the landing, due to this a very high energy absorber material needs to be develop in order to absorb this energy before it can be controlled. This is the challenging task for the material engineer to develop a new material that can absorb high energy at a very short of time.

Finally, it is the best when the real experimental work can be carried out but the issues of the aircraft itself, the length of the linear generator and the regulations on the power grid have limited the works. In doing so, a proper paper works need to be developed where the aircraft industries, the researchers and the policy maker can join together in order to explore this new kind of alternative energy source for the future. 


\section{References}

[1] D. of Energy \& Climate Change, "First progress report on the promotion and use of energy from renewable sources for the united kingdom." [Access on 4 April 2012]:http://www.decc.gov.uk/en/content/cms/meeting_energy/renewable_ener.

[2] W. R. Institute, "Temperature increases," 2012. [Access on 4 April 2012]:http://www.wri.org/publication/content/7787.

[3] S. Zulkifli and M. Ahmad, "Linear generator models in simulink block," in Proc. of The IEEE International Conference on Power and Energy (PECon), pp. 226-231, 2010.

[4] A. R. Mohamed and K. T. Lee, "Energy for sustainable development in malaysia: Energy policy and alternative energy," Energy Policy, vol. 34, no. 15, pp. 2388 2397, 2006.

[5] A. Jolley, "Technologies for alternative energy:climate change working paper no. 7," tech. rep., Melbourne,AUSTRALIA, 2006.

[6] U. Massachusetts Institute of Technology, "Harnessing kinetic energy," 2007. [Access on 11 Sept. 2012]:http://www.technologyreview.com/news/409115/harnessing-kineticenergy.

[7] S. Cholula, A. Claudio, and J. Ruiz, "Intelligent control of the regenerative braking in an induction motor drive," in Proc. of The 2nd International Conference on Electrical and Electronics Engineering, 2005.

[8] B. Cao, Z. Bai, and W. Zhang, "Research on control for regenerative braking of electric vehicle," in Proc. of the IEEE International Conference on Vehicular Electronics and Safety, 2005, 2005.

[9] Q.-C. Zhong, "Ground-operated energy recovery system for landing aircraft," in Proc. of The International Conference on Sustainable Power Generation and Supply, (SUPERGEN), pp. 1-5, 2009. 
[10] P. Steward, Q. Zhong, and M. Eftekhari, "Feasibility study of energy recovery from landing aircraft," 2011. [Access on 11 Sept. 2012]:http://gow.epsrc.ac.uk/NGBOViewGrant.aspx?GrantRef=EP/H004351/2.

[11] J. Shek, D. Macpherson, and M. Mueller, "Control of linear electrical generators for direct drive wave energy conversion." Jun.

[12] L. Su, G. Li, and Z. Jin, "Modeling, control and testing of a voltage-sourceinverter-based microgrid," in Proc. of The 4th International Conference on Electric Utility Deregulation and Restructuring and Power Technologies (DRPT), pp. 724$729,2011$.

[13] L. Xu and P. Cartwright, "Direct active and reactive power control of DFIG for wind energy generation," IEEE Trans. on Energy Conversion, vol. 21, pp. 750 -758 , Sep. 2006.

[14] S.-K. Kim, E.-S. Kim, and J.-B. Ahn, "Modeling and control of a grid-connected wind/pv hybrid generation system," in Proc. of The IEEE PES Transmission and Distribution Conference and Exhibition, pp. 1202-1207, 2006.

[15] L. Qu and W. Qiao, "Constant power control of DFIG wind turbines with supercapacitor energy storage," IEEE Trans. on Industry Applications, vol. 47, pp. 359367, Jan. 2011.

[16] T. Tafticht, K. Agbossou, and A. Cheriti, "Dc bus control of variable speed wind turbine using a buck-boost converter," in Proc. of The IEEE Power Engineering Society General Meeting, 2006.

[17] M. Molina and P. Mercado, "A new control strategy of variable speed wind turbine generator for three-phase grid-connected applications," in Proc. of The IEEE/PES Transmission and Distribution Conference and Exposition: Latin America, 2008.

[18] Z. Ma, "A sensorless control method for maximum power point tracking of wind turbine generators," in Proc. the 14th European Conference on of Power Electronics and Applications (EPE 2011),, pp. 1-10, 2011.

[19] X. Zheng, L. Li, D. Xu, and J. Platts, "Sliding mode MPPT control of variable speed wind power system," in Proc. of The Asia-Pacific Power and Energy Engineering Conference (APPEEC), pp. 1-4, 2009.

[20] A. Zue and A. Chandra, "Simulation and stability analysis of a $100 \mathrm{kw}$ grid connected LCL photovoltaic inverter for industry," in Proc. of The IEEE Power Engineering Society General Meeting, p. 6, 2006. 
[21] R. Naim, G. Weiss, and S. Ben-Yaakov, " $\mathrm{H}^{\infty}$ control applied to boost power converters," IEEE Trans. Power Electron., vol. 12, pp. 677-683, Jul. 1997.

[22] N. Hamrouni and A. Cherif, "Modelling and control of a grid connected photovoltaic system," Renue des Energies Renouvelables, vol. 10, pp. 335-344, 2007.

[23] F. cheng zhi, F. You-tong, Y. yun yue, and L. Zi-xing, "State space model and simulation of long stator linear synchronous motoring maglev vehicle," in Proc. of The Intelligent Transportation Systems, pp. 1482-1485, 2003.

[24] T. Makino and K. Harada, "On the regenerative braking of DC motors with AC supply," IEEE Trans. on Industry Applications, vol. IA-10, pp. 123-127, Jan. 1974.

[25] Z. Chuanwei, B. Zhifeng, C. Binggang, and L. Jingcheng, "Study on regenerative braking of electric vehicle," in Proc. of The 4th International Power Electronics and Motion Control Conference (IPEMC), pp. 836-839, 2004.

[26] Y. Min, B. Zhifeng, and C. Binggang, "Robust $\mathrm{H} 2 / \mathrm{H}^{\infty}$ control for regenerative braking of electric vehicles," in Proc. of The IEEE International Conference on Control and Automation (ICCA), pp. 1366-1370, 2007.

[27] J. Shek, D. Macpherson, M. Mueller, and J. Xiang, "Reaction force control of a linear electrical generator for direct drivewave energy conversion," IET-RPG, vol. 1, pp. 17-24, Mar. 2007.

[28] I. Ivanova, O. Agren, H. Bernhoff, and M. Leijon, "Simulation of cogging in a 100 $\mathrm{kw}$ permanent magnet octagonal linear generatorfor ocean wave conversion," in Proc. of The International Symposium on Underwater Technology, pp. 345-348, 2004 .

[29] P. Brooking and M. Mueller, "Power conditioning of the output from a linear vernier hybrid permanentmagnet generator for use in direct drive wave energy converters," pp. 673-681, Sep. 2005.

[30] A. H. VanderMeulen, "Novel control of a permanent magnet linear generator for ocean wave energy applications," Master's thesis, Oregon State University, Oregon, USA, 2008.

[31] N. Rahim, E. Mahrous, W. Hew, and K. Nor, "Modeling and simulation of the linear generator PWM multilevel inverter," in Proc. of The National Power Engineering Conference (PECon), pp. 95-98, 2003. 
[32] U. Kollorgen Company, "Lcd series ironcore," 2012. [Access on 6 April 2012]:http://www.kollorgen.com/en-us/products/motors/direct-drive/directdrive-linear/icd-series-ironcore.

[33] S. A.E Fitzgerald, C. Kingsley. Jr, Electric Machinery-Fifth Edition in SI Units. McGraw Hill, 1992.

[34] Z. J. P. Jacek F. Gieras, Linear Synchronous Motors- Transportation and Automation Systems. CRC Press, 2000.

[35] J. Shek, D. Macpherson, and M. Mueller, "Phase and amplitude control of a linear generator for wave energy conversion," in Proc. of the IET Conference on Power Electronics, Machines and Drives (PEMD), pp. 66-70, 2008.

[36] A. Santana, D. Andrade, and A. Villa-Jaen, "Output power of linear generators under reactive control in regular waves," in Proc. of The International Conference on Renewable Energies and Power Quality, 2011.

[37] J. Cui, H. Wang, J. Wan, G. Mu, and J. Yang, "Thrust force control of permanent magnet linear synchronous motor basedon sliding mode variable structure," in Proc. of The IEEE International Conference on Control and Automation (ICCA), pp. 1698-1700, 2007.

[38] Z. Zhou, W. Knapp, J. MacEnri, H. Sorensen, E. Friis Madsen, I. Masters, and P. Igic, "Permanent magnet generator control and electrical system configuration for wave dragon MW wave energy take-off system," in Proc. of The IEEE International Symposium on Industrial Electronics (ISIE), pp. 1580-1585, 2008.

[39] Y.-K. Lo, J.-Y. Lin, and T.-Y. Wu, "Grid-connection technique for a photovoltaic system with power factor correction," in Proc. of The International Conferenceon Power Electronics and Drives Systems (PEDS), pp. 522-525, 2005.

[40] P. Binduhewa, A. Renfrew, and M. Barnes, "Ultracapacitor energy storage for microgrid micro-generation," in Proc. of The 4th IET Conference on Power Electronics, Machines and Drives (PEMD), 2008.

[41] M. J. Z. Mehdi, "Power factor improvement of cascaded buck boost converter." Proc. of The National Power Electronics Conference, 2010.

[42] M. Todorovic, L. Palma, and P. Enjeti, "Design of a wide input range DC-DC converter with a robust power controlscheme suitable for fuel cell power conversion," in Proc. of The 19 Annual IEEE Applied Power Electronics Conference and Exposition (APEC), pp. 374-379, 2004. 
[43] J. Chen, D. Maksimovic, and R. Erickson, "Buck-boost PWM converters having two independently controlled switches," in Proc. of The 32th Annual Power Electronics Specialists Conference, pp. 736-741, 2001.

[44] J. Liang and C. Feng, "Stability improvement of micro-grids with coordinate control of fuel cell and ultracapacitor," in Proc. of The IEEE Power Electronics Specialists Conference (PESC), 2007.

[45] S.-H. Song, S. il Kang, and N. kun Hahm, "Implementation and control of grid connected AC-DC-AC power converter for variable speed wind energy conversion system," in Proc. of The 18th Anuual IEEE Applied Power Electronics Conference and Exposition (APEC), 2003.

[46] R. Esmaili, L. Xu, and D. Nichols, "A new control method of permanent magnet generator for maximum power tracking in wind turbine application," in Proc. of The IEEE Power Engineering Society General Meeting, 2005.

[47] C. W. Tan, T. Green, and C. Hernandez-Aramburo, "An improved maximum power point tracking algorithm with current-mode control for photovoltaic applications," in Proc. of International Conference on Power Electronics and Drives Systems (PEDS), 2005.

[48] Y. Nishida, J. Miniboeck, S. D. Round, and J. W. Kolar, "A new 3-phase buckboost unity power factor rectifier with two independently controlled DC outputs," in Proc. of The 22 Annual IEEE Applied Power Electronics Conference, (APEC), pp. 172-178, 2007.

[49] Y.-J. Lee, A. Khaligh, and A. Emadi, "A compensation technique for smooth transitions in non-inverting buck-boost converter," in Proc. of The 24th Annual IEEE Applied Power Electronics Conference and Exposition (APEC), pp. 608614, 2009 .

[50] M. Ghanem, K. Al-Haddad, and G. Roy, "A new control strategy to achieve sinusoidal line current in a cascade buck-boostconverter," IEEE Trans. Ind. Electron., vol. 43, pp. 441-449, Jun. 1996.

[51] S. Mariethoz, S. Almer, M. Baja, A. Beccuti, A. Patino, D.and Wernrud, J. Buisson, H. Cormerais, T. Geyer, H. Fujioka, U. Jonsson, C.-Y. Kao, G. R. A. Morari, M.and Papafotiou, and P. Riedinger, "Comparison of hybrid control techniques for buck and boost DC-DC converters," IEEE Trans. Control Syst. Technol., vol. 18, pp. 1126-1145, Sep. 2010. 
[52] K. Viswanathan, R. Oruganti, and D. Srinivasan, "Dual-mode control of cascade buck-boost PFC converter," in Proc. of The IEEE 35th Annual Power Electronics Specialists Conference (PESC), pp. 2178-2184, 2004.

[53] P. Midya, K. Haddad, and M. Miller, "Buck or boost tracking power converter," IEEE Power Electronics Letters, vol. 2, pp. 131-134, Dec. 2004.

[54] R.-Y. Kim and J.-S. Lai, "Aggregated modeling and control of a boost-buck cascade converter for maximum power point tracking of a thermoelectric generator," in Proc. of The 23 Annual IEEE Applied Power Electronics Conference and Exposition APEC, 2008.

[55] W. Langyuan, W. Xiaobo, and L. Jiana, "A multi-mode four-switch buck-boost dc/dc converter," in Proc. of The Asia-Pacific Power and Energy Engineering Conference (APPEEC), 2009.

[56] D. Bortis, S. Waffler, J. Biela, and J. Kolar, "25kw 3-phase unity power factor buck boost rectifier with wide input and output range for pulse load applications," in Proc. of The IEEE 34th International Conference on Plasma Science (ICOPS), p. $868,2007$.

[57] J. Zhang, J.-S. Lai, and W. Yu, "Bidirectional DC-DC converter modeling and unified controller with digitalimplementation," in Proc. of The 23 Annual IEEE Applied Power Electronics Conference and Exposition (APEC), pp. 1747-1753, 2008.

[58] A. Abouloifa and F. Giri, "Nonlinear control of buck-boost AC-DC converters: output voltage regulation power factor correction," in Proc. of The American Control Conference, pp. 168-173, 2004.

[59] Y. Ye, M. Kazerani, and V. Quintana, "A novel modeling and control method for three-phase PWM converters," in Proc. of The IEEE 32nd Annual Power Electronics Specialists Conference (PESC), pp. 102-107, 2001.

[60] F. Tahami, H. Ahmadian, and A. Moallem, "Robust $\mathrm{H}^{\infty}$ control design for PFC rectifiers," in Proc. of The 7th International Conference on Power Electronics and Drive Systems, (PEDS)', pp. 948-952, 2007.

[61] M. Gaboriault and A. Notman, "A high efficiency, noninverting, buck-boost DCDC converter," in Proc. of The 19th Annual IEEE Applied Power Electronics Conference and Exposition, pp. 1411-1415, 2004. 
[62] L. Barote and C. Marinescu, "Storage analysis for stand-alone wind energy applications," in Proc. of The 12th International Conference on Optimization of Electrical and Electronic Equipment (OPTIM), pp. 1180-1185, 2010.

[63] W. Li, G. Joos, and C. Abbey, "A parallel bidirectional DC/DC converter topology for energy storage systems in wind applications," in Proc. of The 42nd IAS Annual Meeting Conference Record of the Industry Applications Conference, pp. 179-185, 2007.

[64] X. Gao, S. Wang, and T. Wei, "Energy management method of supercapacitors storage system for UPS applications," in Proc. of The International Conference on Applied Superconductivity and Electromagnetic Devices (ASEMD), pp. 68-72, 2009 .

[65] R. Aghatehrani, R. Kavasseri, and R. Thapa, "Power smoothing of the dfig wind turbine using a small energy storage device," in Proc. of The IEEE Power and Energy Society General Meeting, 2010.

[66] K. Jin, X. Ruan, M. Yang, and M. Xu, "A hybrid fuel cell power system," IEEE Trans. Ind. Electron., vol. 56, pp. 1212-1222, Apr. 2009.

[67] P. Thounthong, S. Rael, and B. Davat, "Control strategy of fuel cell and supercapacitors association for a distributed generation system," IEEE Trans. Ind. Electron., vol. 54, pp. 3225-3233, Dec. 2007.

[68] P. Thounthong, S. Rael, and B. Davat, "Control algorithm of fuel cell and batteries for distributed generation system," IEEE Trans. on Energy Conversion, pp. 148155, Mac. 2008.

[69] A. S. Samosir and A. H. M. Yatim, "Dynamic evolution control for synchronous buck DC/DC converter: Theory, model and simulation," Simulation Modelling Practice and Theory, vol. 18, no. 5, pp. 663-676, 2010.

[70] X. del Toro Garcia, P. Roncero-Sanchez, A. Parreno, and V. Feliu, "Ultracapacitorbased storage: Modelling, power conversion and energy considerations," in Proc. of The IEEE International Symposium on Industrial Electronics (ISIE), pp. 2493$2498,2010$.

[71] L. Fernandez, C. Garcia, P. Garcia, J. Torreglosa, and F. Jurado, "Control strategies of a fuel-cell hybrid tramway integrating two dc/dc converters," in Proc. of The International Symposium on Power Electronics Electrical Drives Automation and Motion (SPEEDAM), pp. 1052-1057, 2010. 
[72] M.-Y. Ayad, S. Pierfederici, S. Raë, and B. Davat, "Voltage regulated hybrid DC power source using supercapacitors as energy storage device," Energy Conversion and Management, vol. 48, pp. 2196-2202, 2007.

[73] A. Payman, S. Pierfederici, and F. Meibody-Tabar, "Energy control of supercapacitor/fuel cell hybrid power source," Energy Conversion and Management, vol. 49, pp. 1637-1644, 2008.

[74] Z. Guoju, T. Xisheng, and Q. Zhiping, "Research on battery supercapacitor hybrid storage and its application in microgrid," in Proc. of The Asian-Pacific on Power and Energy Engineering Conference (APPEEC), pp. 1-4, 2010.

[75] A. Khaligh and Z. Li, "Battery, ultracapacitor, fuel cell, and hybrid energy storage systems for electric, hybrid electric, fuel cell, and plug-in hybrid electric vehicles: State of the art," IEEE Trans. on Vehicular Technology, vol. 59, pp. 2806-2814, july 2010.

[76] "Electronic power transformer with supercapacitors storage energy system," Electric Power Systems Research, vol. 79, no. 8, pp. 1200-1208, 2009.

[77] B. Chen, Y. Gao, M. Ehsani, and J. Miller, "Design and control of a ultracapacitor boosted hybrid fuel cell vehicle," in Proc. of The IEEE Vehicle Power and Propulsion Conference (VPPC), 2009.

[78] A. Samosir and A. Yatim, "Implementation of dynamic evolution control of bidirectional DC -DC converterfor interfacing ultracapacitor energy storage to fuelcell system," IEEE Trans. Ind. Electron., vol. 57, pp. 3468-3473, Oct. 2010.

[79] B. Narasimharaju, S. Dubey, and S. Singh, "Voltage mode control of coupled inductor bidirectional DC to DC converter," in Proc. of The IEEE International Conference of Electron Devices and Solid-State Circuits (EDSSC), 2010.

[80] M. Ortuzar, J. Dixon, and J. Moreno, "Design, construction and performance of a buck-boost converter for an ultracapacitor-based auxiliary energy system for electric vehicles," in Proc. of The 29th Annual Conference of the IEEE Industrial Electronics Society (IECON), 2003.

[81] P. Grbović and, P. Delarue, P. Le Moigne, and P. Bartholomeus, "The ultracapacitor-based controlled electric drives with braking and ride-through capability: Overview and analysis," IEEE Trans. on Industrial Electronics, vol. 58, pp. 925-936, march 2011. 
[82] C. Nwosu, "State-space averaged modeling of a nonideal boost converter," The Pacific Journal of Science and Technology, vol. 9, p. 302, Nov. 2008.

[83] S. Zulkifli., "Energy recovery from landing aircraft." to be published, 2012.

[84] M. Amin, M. Elshaer, and O. Mohammed, "Dc bus voltage control for pv sources in a dc distribution system infrastructure," in Proc. of The IEEE Power and Energy Society General Meeting, 2010.

[85] M. Elshaer, A. Mohamed, and O. Mohammed, "Smart optimal control of dc-dc boost converter for intelligent pv systems," in Proc. of The 16th International Conference on Intelligent System Application to Power Systems (ISAP), 2011.

[86] J. Mahdavi, A. Emadi, and H. Toliyat, "Application of state space averaging method to sliding mode control of PWM DC/DC converters," in Proc. of The 32th IAS Annual Meeting on Conference Record Industry Applications Conference (IAS), pp. 820-827, 1997.

[87] H. Guldemir, "Sliding mode control of DC-DC boost converter," Journal of Applies Sciences, vol. 5 (3), pp. 588-592, 2005.

[88] P. Donoso-Garcia, P. Cortizo, B. de Menezes, and M. Severo Mendes, "Slidingmode control for current distribution in parallel-connected DC-DC converters," IEE Proc. on Electric Power Applications, vol. 145, pp. 333-338, Jul. 1998.

[89] T. Hornik, Power Quality in Microgrids. PhD thesis, University of Liverpool, Liverpool,UK, October 2010.

[90] M. Liserre, R. Teodorescu, and F. Blaabjerg, "Stability of grid-connected PV inverters with large grid impedance variation," in Proc. of The IEEE 35th Annual Power Electronics Specialists Conference (PESC), pp. 4773-4779, 2004.

[91] M. Liserre, F. Blaabjerg, and S. Hansen, "Design and control of an LCL-filterbased three-phase active rectifier," IEEE Trans. on Industry Applications, vol. 41, pp. 1281-1291, Sep. 2005.

[92] E. Twining and D. Holmes, "Grid current regulation of a three-phase voltage source inverter with an lcl input filter," IEEE Trans. on Power Electronics, pp. 888-895, May 2003.

[93] N. Hamrouni and A. Cherif, "New method of current control for LCL interfaced grid connected three phase voltage source inverter," Renue des Energies Renouvelables, vol. 13, 2010. 
[94] T. Hornik and Q.-C. Zhong, "Control of grid-connected DC-AC converters in distributed generation: Experimental comparison of different schemes," in Proc. of The Compatibility and Power Electronics (CPE), pp. 271 -278, May.

[95] C. Picardi and D. Sgro, "Grid-connected inverter power flow control based on a new modeling approach of electrical signals," in Proc. of The International Conference on Clean Electrical Power, pp. 585-590, 2009.

[96] I. Hiskens and E. Fleming, "Control of inverter-connected sources in autonomous microgrids," in Proc. of The American Control Conference, pp. 586 -590, 2008.

[97] A. Timbus, R. Teodorescu, F. Blaabjerg, M. Liserre, and P. Rodriguez, "Linear and nonlinear control of distributed power generation systems," in Proc. of The 41st IAS Annual Meeting Conference Record on Industry Applications Conference, pp. 1015-1023, 2006.

[98] Z. Ma, "Synchronverter: Direct power control for three phase PWM rectifiers." to be published, Aug. 2012.

[99] L. P. Nyuen, "Design of a non-pll synchronverter-like statcom controller." to be published, Jan 2012.

[100] T. Hornik and Q.-C. Zhong, "A current-control strategy for voltage-source inverters in microgrids based on $\mathrm{H}^{\infty}$ and repetitive control," IEEE Trans. Power Electron.

[101] G. Weiss, Q.-C. Zhong, T. Green, and J. Liang, "H repetitive control of DC-AC converters in microgrids," IEEE Trans. Power Electron., vol. 19, pp. 219-230, Jan. 2004.

[102] T. Hornik and Q.-C. Zhong, " $\mathrm{H}^{\infty}$ repetitive voltage control of gridconnected inverters with a frequency adaptive mechanism," Power Electronics, IET, vol. 3, pp. 925-935, Nov. 2010.

[103] R. Teodorescu, F. Blaabjerg, U. Borup, and M. Liserre, "A new control structure for grid-connected lcl pv inverters with zero steady-state error and selective harmonic compensation," in Proc. of The 19th Annual IEEE on Applied Power Electronics Conference and Exposition (APEC), 2004.

[104] L. Shang, D. Sun, and J. Hu, "Sliding-mode-based direct power control of gridconnected voltage-sourced inverters under unbalanced network conditions," IET Trans. on Power Electronics, vol. 4, pp. 570-579, May. 2011. 
[105] L. Serpa, J. Kolar, S. Ponnaluri, and P. Barbosa, "A modified direct power control strategy allowing the connection of three-phase inverter to the grid through LCL filters," in Proc. of The 40 IAS Annual Meeting.Conference Record of the Industry Applications Conference, vol. 1, pp. 565-571, 2005.

[106] T. Noguchi, H. Tomiki, S. Kondo, and I. Takahashi, "Direct power control of PWM converter without power-source voltage sensors," IEEE Trans. on Industry Applications, vol. 34, pp. 473-479, May/Jun 1998.

[107] G. Raducu, "Control of grid side inverter in a B2B configuration for WT applications," Master's thesis, Aalborg University, Aalborg, Denmark, 2008.

[108] Q.-C. Zhong and P.-L. Nguyen, "Sinusoid-locked loops based on the principles of synchronous machines," in Proc. of The 24th Chinese Control and Decision Conference (CCDC), pp. 1518-1523, 2012.

[109] E. Vidal-Idiarte, L. Martinez-Salamero, H. Valderrama, and F. Guinjoan, " $\mathrm{H}^{\infty}$ control of DC-to-DC switching converters," in Proc. of The IEEE International Symposium on Circuits and Systems (ISCAS), pp. 238-241, 1999.

[110] R. wen Yu, H. Zhang, and G. jun Tan, "Design and implementation of $\mathrm{H}^{\infty}$ controller for three-phase PWM rectifiers," in Proc. of The International Conference on Computer Application and System Modeling (ICCASM), pp. V8-69-V8-72, 2010.

[111] F.-J. Lin, T.-S. Lee, and C.-H. Lin, "Robust $\mathrm{H}^{\infty}$; controller design with recurrent neural network for linear synchronous motor drive," IEEE Trans. on Industrial Electronics, vol. 50, pp. 456-470, june 2003.

[112] U. Supatti, S. Boonto, C. Prapanavarat, and V. Moneyakul, "Design of an $\mathrm{H}^{\infty}$ robust controller for multi-module parallel DC-DC buck converters with average current mode control," in Proc. of The IEEE International Conference on Industrial Technology (ICIT), pp. 992-997, 2002.

[113] R. Naim, G. Weiss, and S. Ben-Yaakov, " $\mathrm{H}^{\infty}$ control of boost converters: comparison to voltage mode, feedforwardand current mode controls," in Proc. of The 26th Annual IEEE Power Electronics Specialists Conference: Record, pp. 1327-1332, 1995.

[114] Y. W. Li, D. Mahinda Vilathgamuwa, F. Blaabjerg, and P. ChiangLoh, "A robust control scheme for medium-voltage-level DVR implementation," IEEE Trans. Ind. Electron., vol. 54, pp. 2249-2261, Aug. 2007. 
[115] T.-S. Lee, S.-J. Chiang, and J.-M. Chang, "H${ }^{\infty}$ loop-shaping controller designs for the single-phase UPS inverters," IEEE Trans. on Power Electronics, pp. 473-481, Jul. 2001.

[116] J. Treurnicht, "H control," May 2007. [Access on October 2011].

[117] J. B. Burl, Linear Optimal Control:H2/H . Addison Wesley Logman, 1st ed., 1999.

[118] K. Zhou, Robust and optimal control. Upper Saddle River, N.J : Prentice Hall, 1996.

[119] M. Yazdanpanah, E. Semsar, and B. Siahkolah, "An $\mathrm{H}^{\infty}$ robust controller for single- phase PWM inverters," in Proc. of The 15th IFAC Triennial World Congress Barcelona, Spain, 2002.

[120] K. H. Kwan, Y. C. Chu, and P. L. So, "Model-based $\mathrm{H}^{\infty}$ control of a unified power quality conditioner," IEEE Trans. on Industrial Electronics, vol. 56, pp. 24932504, Jul. 2009.

[121] M. Aten and H. Werner, "Robust multivariable control design for HVDC back to back schemes," IEE Proc. on Generation, Transmission and Distribution, vol. 150, pp. 761-767, Nov. 2003.

[122] G. Collins, L. Nguyen, S. Phillips, B. Bakkaloglu, and D. Runton, "H-infinity robust design methodology approach to RF PA linearization," in Proc. of 36th European Microwave Conference, 2006.

[123] O. Sename, Robust Control: Analysis and Design. [Access on Disember 2011]:http://www.gipsalab.grenobleinp.fr/o.sename/docs/RobustControl.pdf.

[124] U. of Strathclyde, "Household electricity consumption," 2012. [Access on 8 April 2012]:http://www.esru.strath.ac.uk/EandE/Websites/01-02/REinfo/hec.htm.

[125] W. D. John J. Grainger, Power System Analysis. McGraw Hill, 1994.

[126] H. Saadat, Power System Analysis. McGraw-Hill, 1999.

[127] J. Gong, R. Xie, W. Liu, and S. Xie, "H${ }^{\infty}$ controller design of the synchronous generator," in Proc. of The International Conference on Intelligent Computation Technology and Automation (ICICTA), vol. 1, pp. 370-374, 2008.

[128] E. Mouni, S. Tnani, and G. Champenois, "Synchronous generator output voltage real-time feedback control via $\mathrm{H}^{\infty}$ strategy," IEEE Trans. Energy Convers., vol. 24, pp. 329-337, Jun. 2009. 
[129] J. Lu and W. Ma, "Modeling, simulation and testing of linear permanent magnet brushless dc motor," 2009.

[130] Y.-R. Chen, N. Cheung, and J. Wu, " $\mathrm{H}^{\infty}$ robust control of permanent magnet linear synchronous motor inhigh-performance motion system with large parametric uncertainty," in Proc. of The 33rd Annual Power Electronics Specialists Conference, pp. 535-539, 2002.

[131] A. Dehkordi, A. Gole, and T. Maguire, "Permanent magnet synchronous machine model for real time simulation," in Proc. on The International Conference on Power Systems Transient (IPST), 2005.

[132] Q. Zhong and G. Weiss, "Synchronverters: Inverters that mimic synchronous generators," IEEE Trans. Ind. Electron., vol. 58, pp. 1259-1267, Apr. 2010.

[133] Z. Salam, "Drives and system," 2001.

[134] S. Nair, "Automatic weight selection algorithm for designing $\mathrm{H}^{\infty}$ controller for active magnetic bearing," International Journal of Engineeing Science and Technology, vol. 3, pp. 122-136, Jan. 2011.

[135] V. Blasko, "Power conditions and control of a regenerative brake," in Proc. of The 33th IAS Annual Meeting The Industry Applications Conference, pp. 1504-1510, 1998.

[136] P. J. Grbovic, P. Delarue, P. Le Moigne, and P. Bartholomeus, "Modelling and control of the ultra-capacitor based regenerative controlled electric drives," IEEE Trans. Ind. Electron., vol. 58, pp. 3471-3484, Aug. 2010.

[137] A. Rufer and P. Barrade, "A supercapacitor-based energy storage system for elevators with soft commutated interface," in Proc. of The 36th IAS Annual Meeting IEEE Industry Applications Conference, 2001.

[138] S. Araujo, A. Engler, B. Sahan, and F. Antunes, "LCL filter design for gridconnected NPC inverters in offshore wind turbines," in Proc. of the 7th Internatonal Conference on Power Electronics (ICPE), pp. 1133-1138, 2007.

[139] R. Banks, J. brinkley, R. Allnutt, and R. Harding, "Human response to acceleration," in Fundamentals of Aerospace Medicne, 2008. 


\section{Appendix A}

\section{Publication papers and test rig schematic diagram}

\section{A.1 List of publications}

1. S.A.Zulkifli, M.Z. Ahmad, "Linear generator models in Simulink," in Proc. of IEEE International Conference on Power and Energy (PECon), 2010.

2. S.A.Zulkifli, "Energy Recovery from Landing Aircraft," in Proc. of The IEEE 3rd International Symposium on Power Electronics for Distributed Generation Systems (PEDG), 2012.

3. S.A.Zulkifli,M.Z.Ahmad, E.Sulaiman "Control of Power Generated During Landing Aircraft," in Proc. of the 7th IEEE Conference on Industrial Electronics and Applications (ICIEA), 2012. 
A.2 The schematic diagram for the test rig development

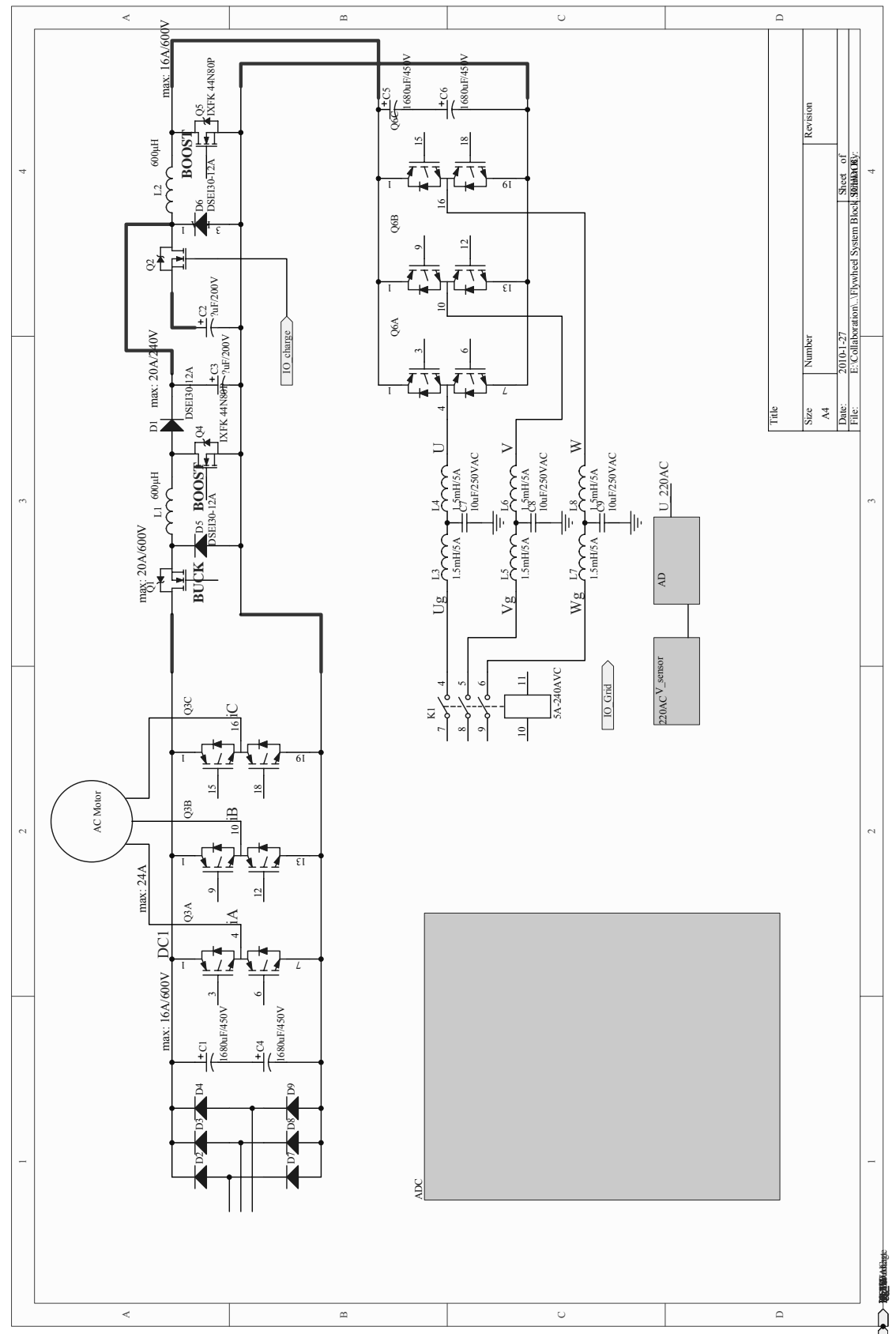

Figure A.1: Schematic diagram for the rig 


\section{Appendix B}

\section{Simulations test with different LCL value}

The simulations have been carried in order to get the better output results for the ERLA. This is done by changing the $L_{i}$ inverter inductance that is given table below:

Table B.1: With the different value for LCL filter

\begin{tabular}{|c|c|c|c|}
\hline Parameters & value & Parameters & value \\
\hline \hline$R_{i}$ & $0.04 \Omega$ & $L_{i}$ & $10 \mathrm{mH}$ \\
\hline$R_{g}$ & $0.1 \Omega$ & $L_{g}$ & $15 \mathrm{uH}$ \\
\hline$R_{c}$ & $10 \Omega$ & $C$ & $5.5 \mathrm{uF}$ \\
\hline
\end{tabular}

The new compensator value $K$ which has been determined due to the LCL filter is given by:

$$
K=H^{\infty}=\frac{202(s+102.1)}{(s+313.4)}
$$

\section{B.1 With the reference power of $P^{*}=1000 \mathrm{~W}$ and reactive of $Q^{*}=0 \mathrm{VAR}$}

The results for the different LCL value for the first case are given in Figure B.1, B.2 and B.3. 


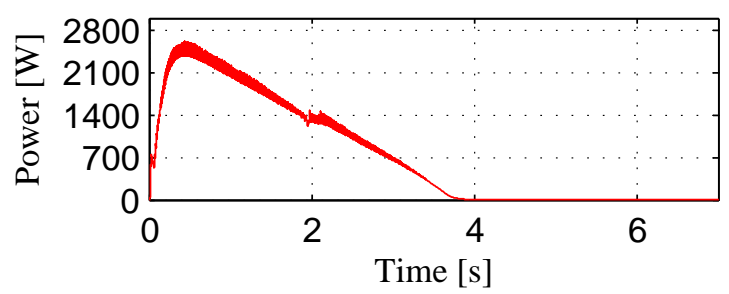

(a) generated output at LG

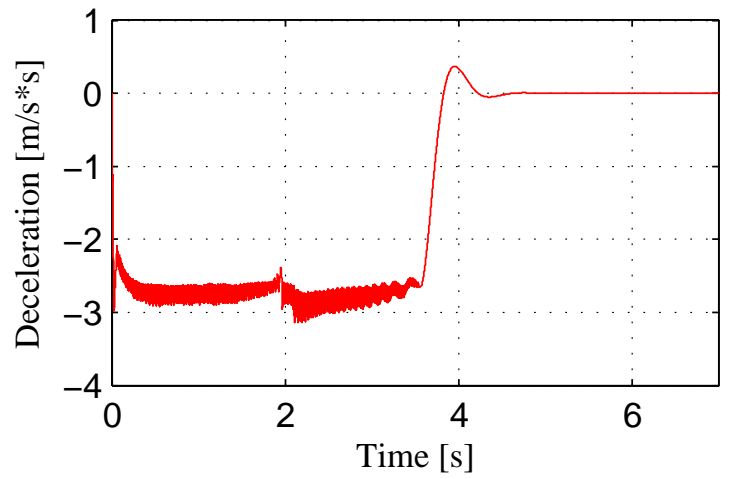

(b) deceleration output at LG

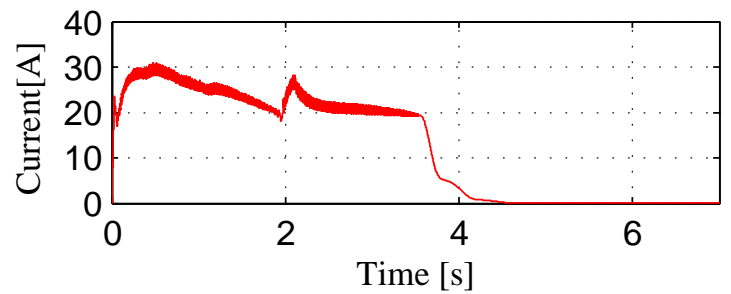

(c) inductor current in $\mathrm{CBB}$ converter

Figure B.1: Outputs generated at the energy conversion

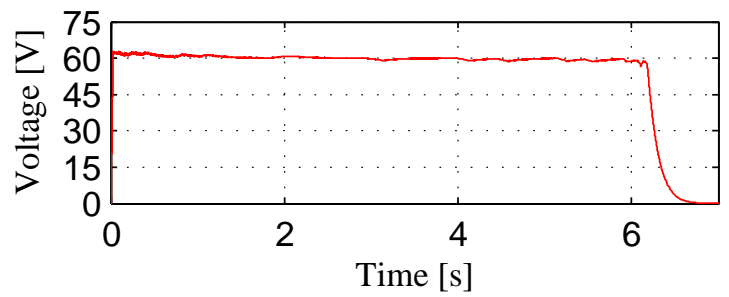

(a) dc-link voltage

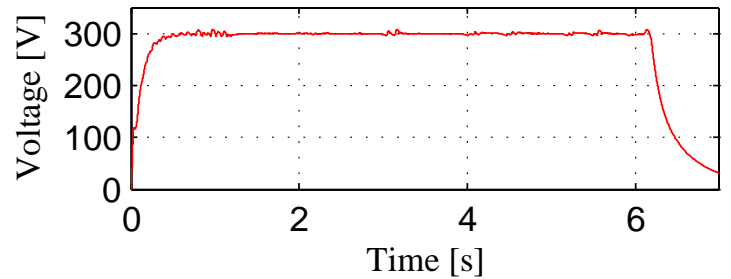

(b) boost converter output or input volage to inverter

Figure B.2: Outputs generated at the energy storage 


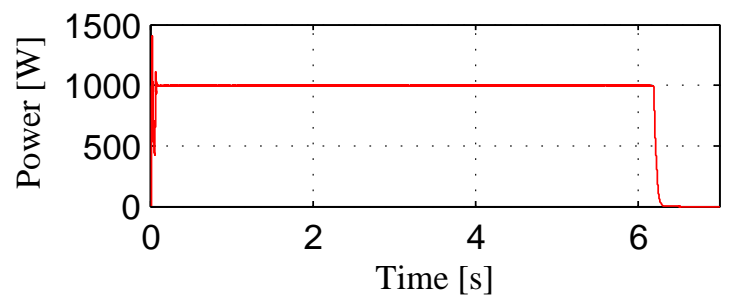

(a) real power transfer to the grid

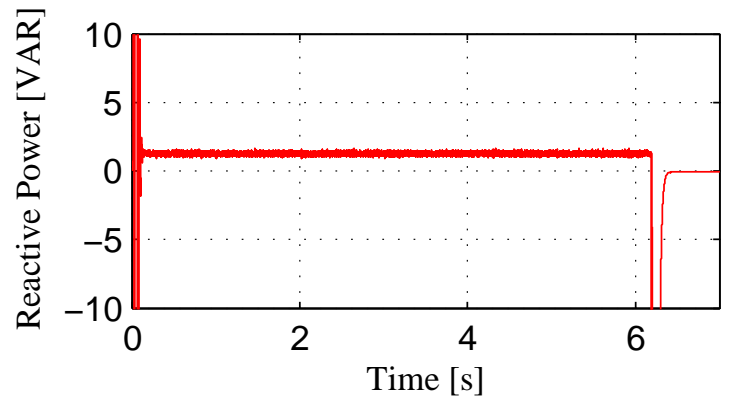

(b) reactive power transfer to the grid

Figure B.3: Outputs generated at the energy transfer 
B.2 With $P^{*}=500 \mathrm{~W}$ from $t=0$ s to $t=3$ s and with real $P^{*}=1000 \mathrm{~W}$ after $t=3 \mathrm{~s}$ and $Q^{*}=0 \mathbf{V A R}$

The results for the different LCL value for the first case are given in Figures B.4, B.5, B.6.

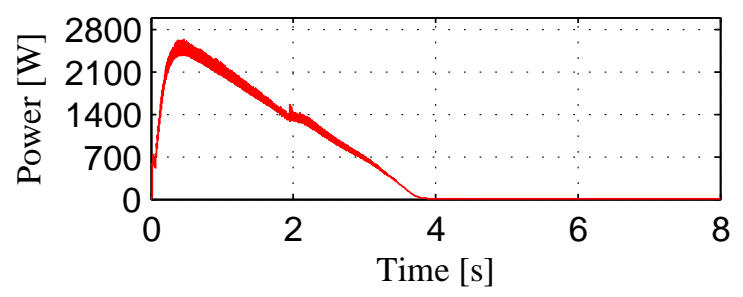

(a) generated power at LG

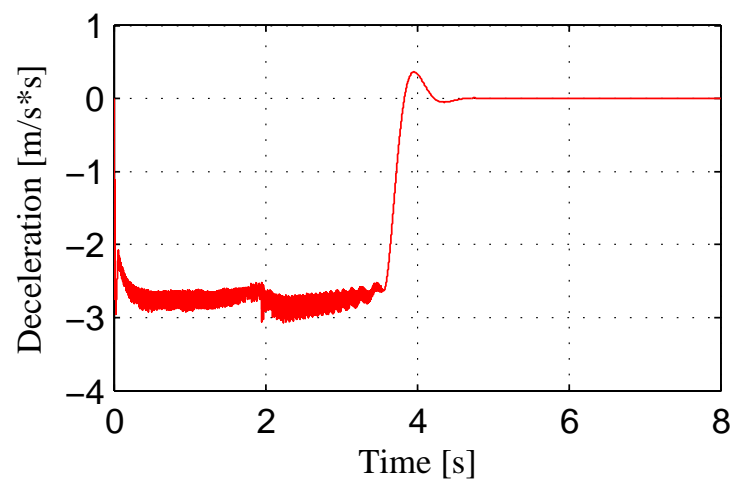

(b) deceleration for LG

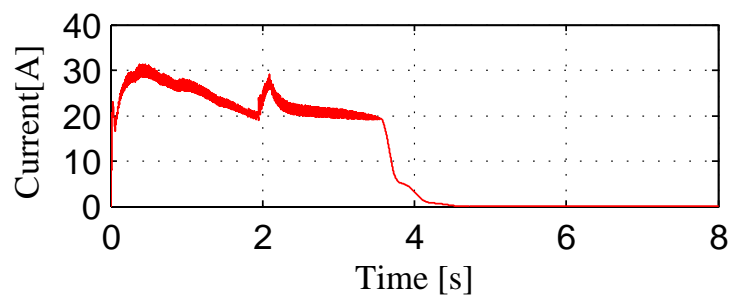

(c) inductor current at $\mathrm{CBB}$ converter

Figure B.4: Outputs generated at energy conversion 


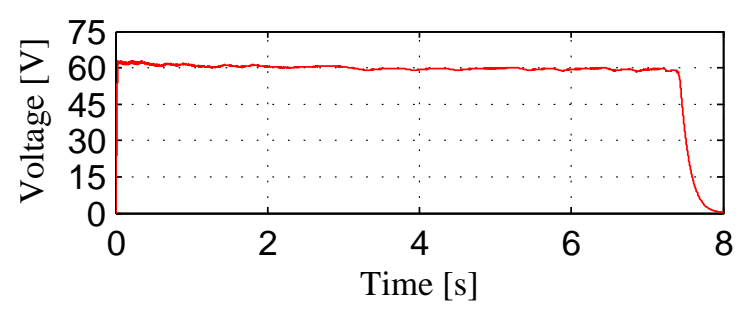

(a) dc-link voltage

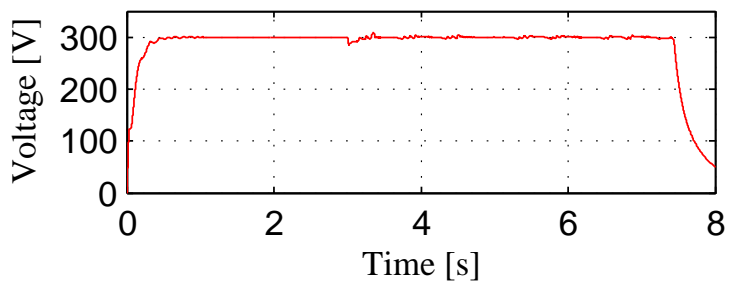

(b) boost converter output or input volage to inverter

Figure B.5: Outputs generated at the energy storage

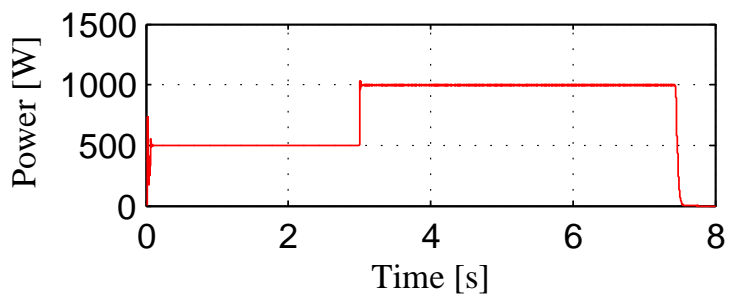

(a) real power transfer to the grid

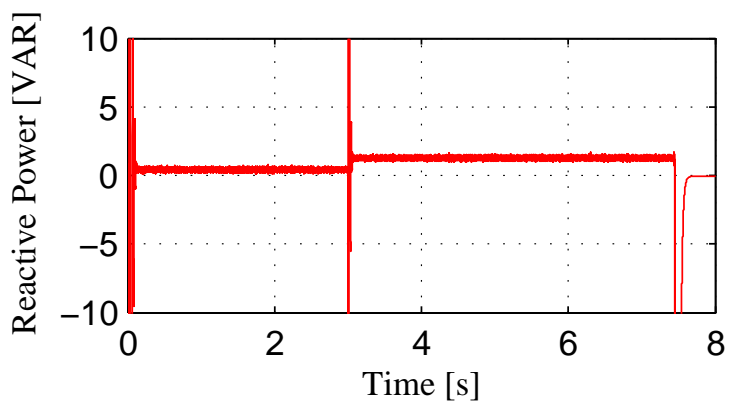

(b) reactive power transfer to the grid

Figure B.6: Outputs generated at the energy transfer 


\section{Appendix C}

\section{List of symbols}

\begin{tabular}{|c|c|}
\hline symbols & unit \\
\hline \hline$v$ & speed $\left(\frac{m}{s}\right)$ \\
\hline$f$ & $\operatorname{Hz}$ \\
\hline$\beta$ & $\sin (\omega t)$ \\
\hline$p$ & watt $\left(\frac{\text { Joule }}{s}\right)$ \\
\hline$E$ & Joule \\
\hline$R$ & $\Omega$ \\
\hline$C$ & $u F$ \\
\hline$L$ & $m H$ \\
\hline$P$ & real power $(\mathrm{W})$ \\
\hline$Q$ & reactive power $(\mathrm{VAR})$ \\
\hline$t$ & time $(\mathrm{s})$ \\
\hline$m$ & mass $(\mathrm{kg})$ \\
\hline$a$ & acceleration $\left(\frac{m}{s^{2}}\right)$ \\
\hline$\omega$ & $\frac{2 \pi}{t}$ \\
\hline$F, F_{e}, F_{m}$ & newton $(\mathrm{N})$ \\
\hline$\theta$ & phase angle $\left({ }^{0}\right)$ \\
\hline$P_{a v g}$ & average power $(\mathrm{W})$ \\
\hline$V_{d c}$ & dc-link voltage $(\mathrm{V})$ \\
\hline$I_{L_{b b}}$ & ampere $(\mathrm{A})$ \\
\hline$I_{s}, I_{R}, I_{C_{c b b}}$ & ampere $(\mathrm{A})$ \\
\hline$V_{s}, V_{0}$ & voltage $(\mathrm{V})$ \\
\hline & \\
\hline
\end{tabular}

